\title{
CYTOFLOW OPTICS ANALYSIS
}

\author{
A Thesis \\ presented to \\ the Faculty of California Polytechnic State University, \\ San Luis Obispo
}

\author{
In Partial Fulfillment \\ of the Requirements for the Degree \\ Master of Engineering in Biomedical field
}

by

Matthew Wayne Janke

March 2015 
@ 2015

Matthew Wayne Janke

ALL RIGHT RESERVED 


\section{COMMITTEE MEMBERSHIP}

TITLE: Cytoflow Optics Analysis

AUTHOR: $\quad$ Matthew Wayne Janke

DATE SUBMITTED: March 2015

COMMITTEE CHAIR: Lily Laiho, Ph.D.

Associate Professor of Biomedical Engineering

COMMITTEE MEMBER: Richard Savage, Ph.D.

Director Graduate Education

COMMITTEE MEMBER: Michael Whitt, Ph.D.

Lecturer of Biomedical Engineering

COMMITTEE MEMBER: Nathan Bair, MS

CTO of CytoFlow 


\section{ABSTRACT \\ Cytoflow Optics Analysis \\ Matthew Wayne Janke}

This project was founded by Cytoflow a flow cytometry company. The company's aim is to make a custom cytometer that can be used by consumers. The purpose of this project was to create a design for the optics assembly of the cytometer. Prototypes were constructed and then tested to analyze results. Data was collected then analyzed using a specific standard across all cytometers. Using these guidelines a result was yielded that helped to improve the resolution of the system and decrease the size. Making the prototype more efficient and lower the cost. From the design review Cytoflow was able to develop a pathway used to push their project towards the market. 


\section{TABLE OF CONTENTS}

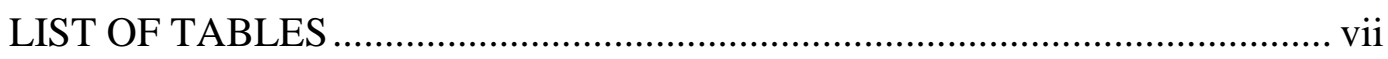

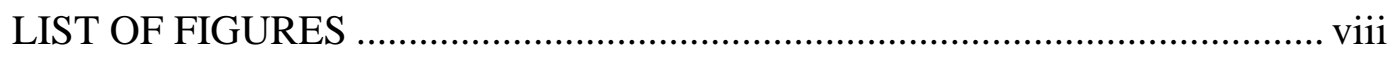

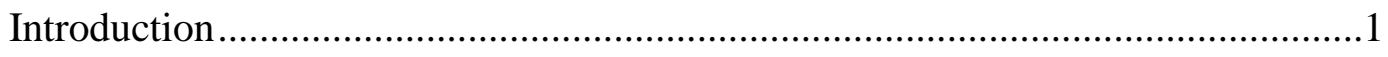

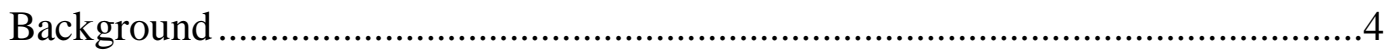

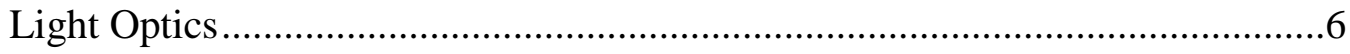

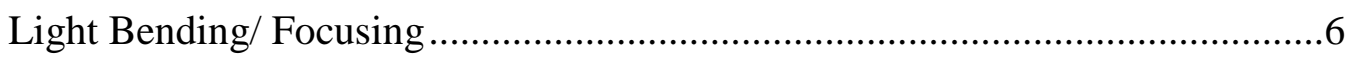

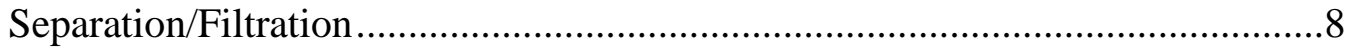

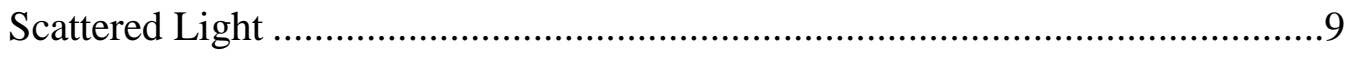

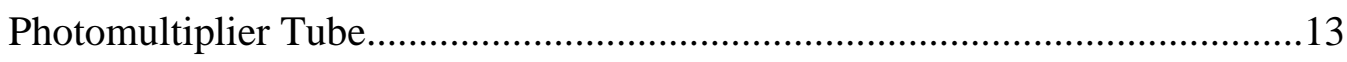

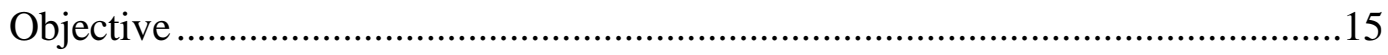

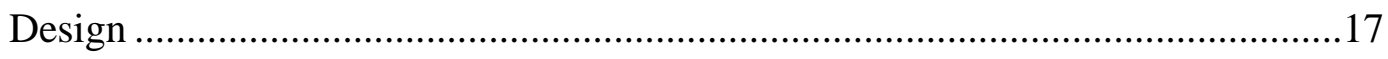

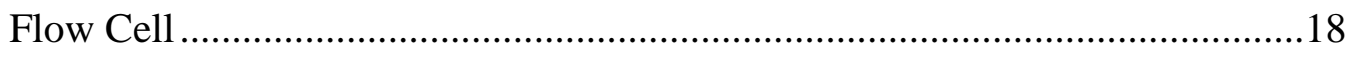

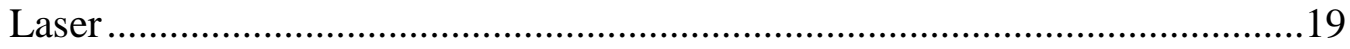

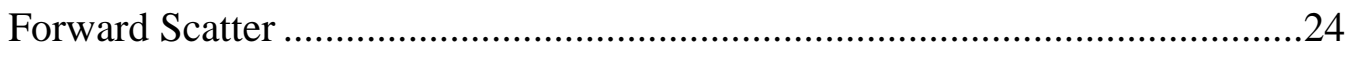

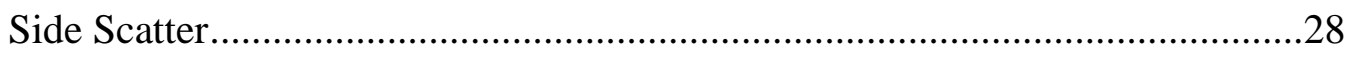

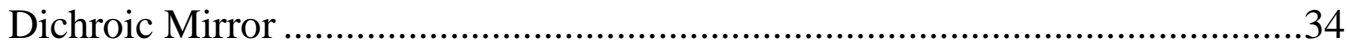

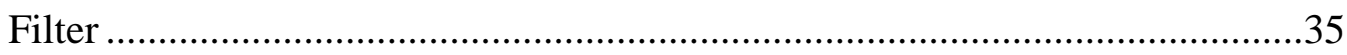

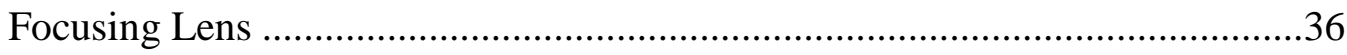

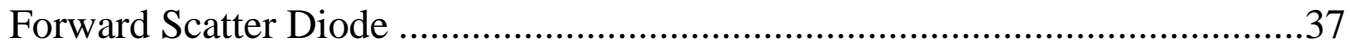

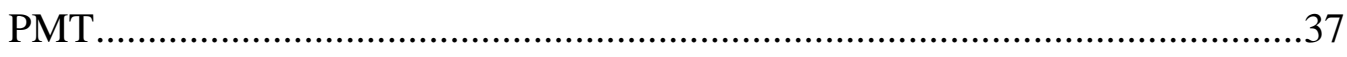

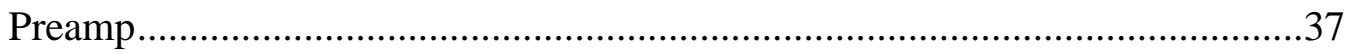

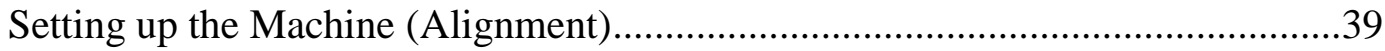

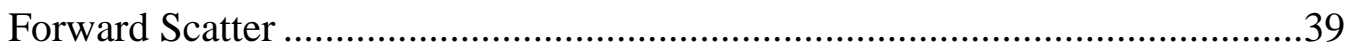

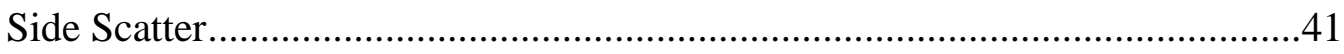

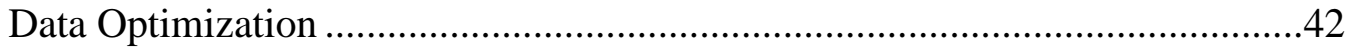

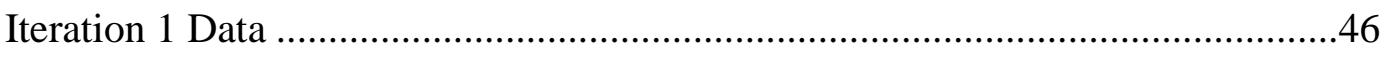

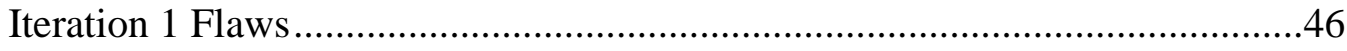

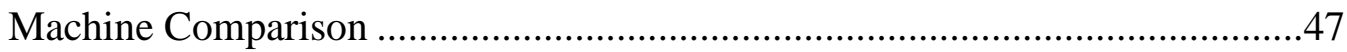

Objective of machine comparison ......................................................47

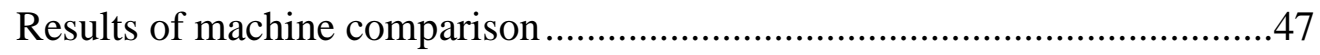




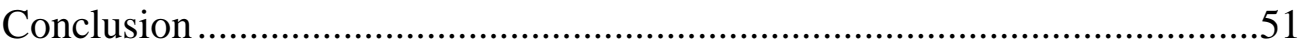

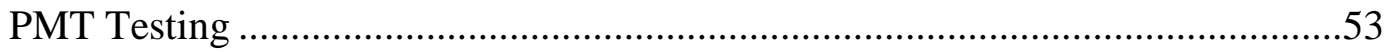

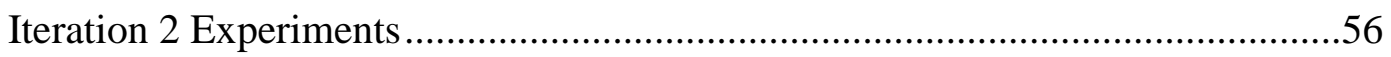

Laser Shape Testing of iteration 2...............................................................56

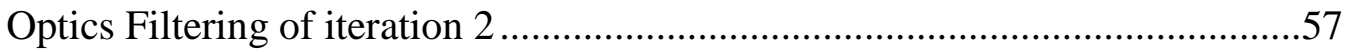

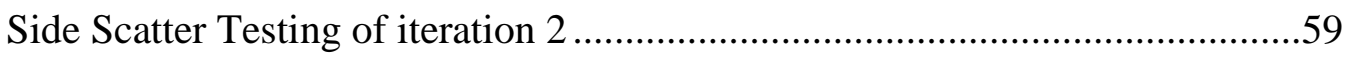

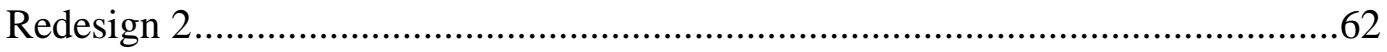

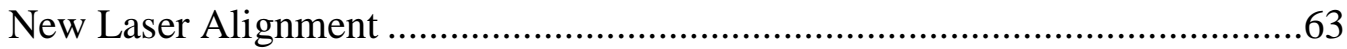

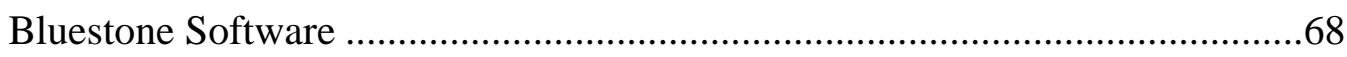

Refinement of the Assembly ............................................................................69

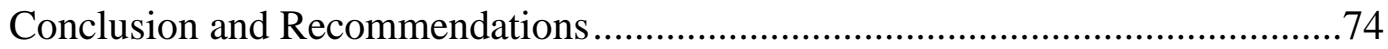

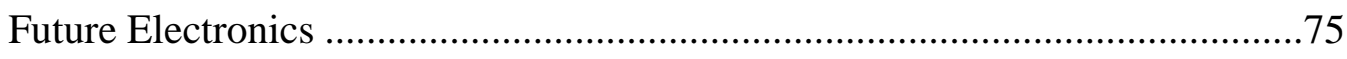

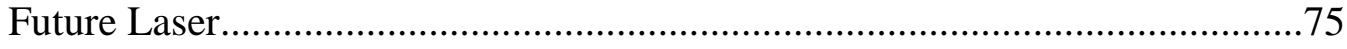

Future Manufacturing ..................................................................................

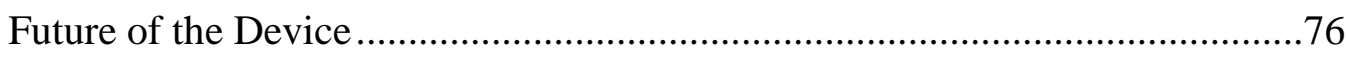

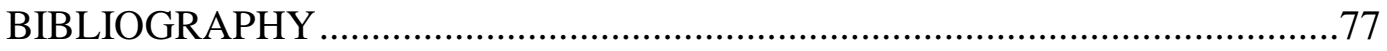

Appendices

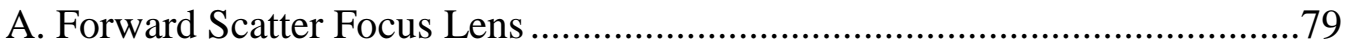

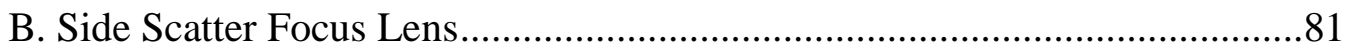

C. Fluorescence Dichroic Mirrors ........................................................................ 83

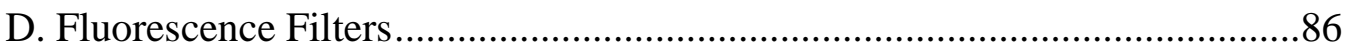

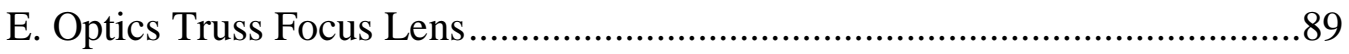

F. Hamamatsu Photomultiplier Tube ……………………………………....91

G. Spherotech Rainbow Calibration Beads .........................................................94

H. Laser Shape Testing Images ...................................................................103

I. Plano Convex Beam Shaping Optic............................................................109

J. Plano Convex Beam Shaping Optic ..........................................................111

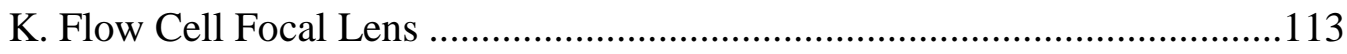

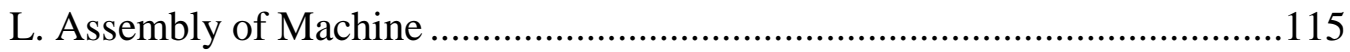




\section{LIST OF TABLES}

Table 1. Application for flow cytometry ........................................................2

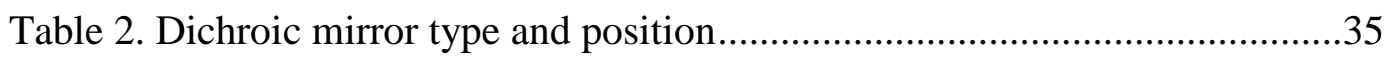

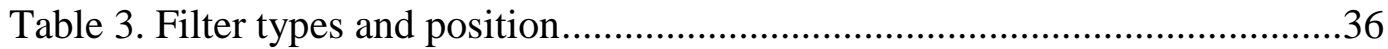




\section{LIST OF FIGURES}

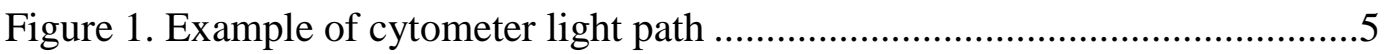

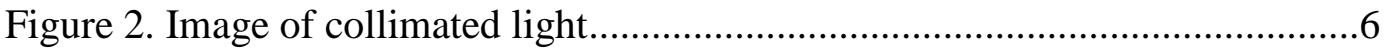

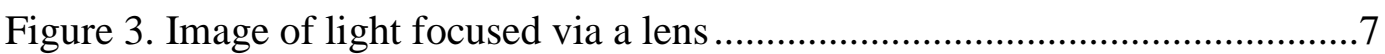

Figure 4. Light being focused then collimated again........................................

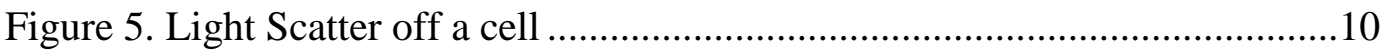

Figure 6. Example image collected from whole blood ..........................................11

Figure 7. Example of how cells are displaced into regions .................................12

Figure 8. Inside of a Photomultiplier Tube ......................................................13

Figure 9. First iteration design with light path in blue.......................................17

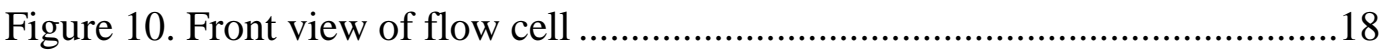

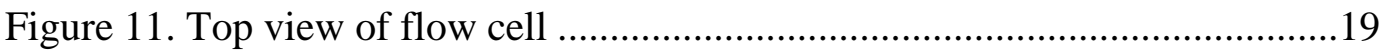

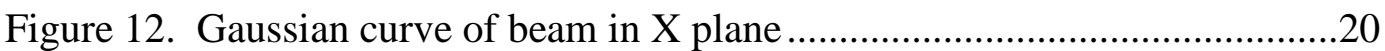

Figure 13. Beam in Y plane with cells passing through .....................................20

Figure 14. BSR Laser projection from top and side view....................................21

Figure 15. BSR Laser projection at $31 \mathrm{~mm}$ from capillary top view (left) and side

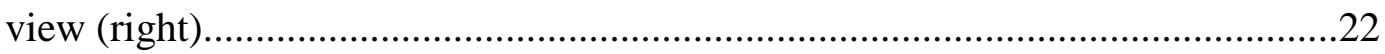

Figure 16. BSR Laser projection at $29 \mathrm{~mm}$ from capillary top view (left) and side

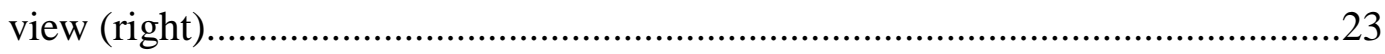

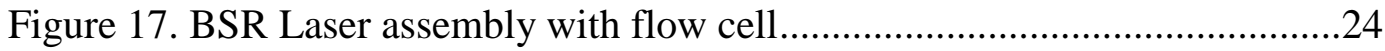

Figure 18. Beam shape passing through a cell..................................................25

Figure 19. Beam shape passing through cell with beam blocker incorporated .....26

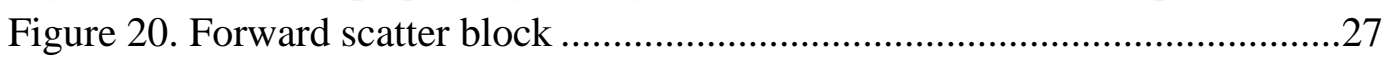

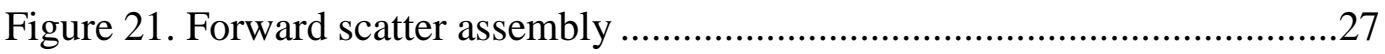

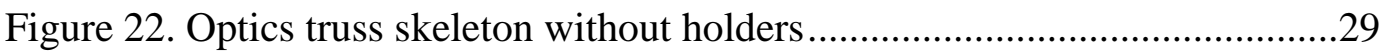

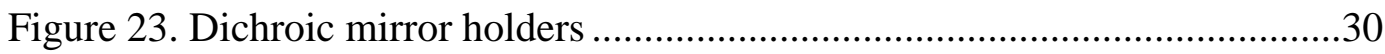

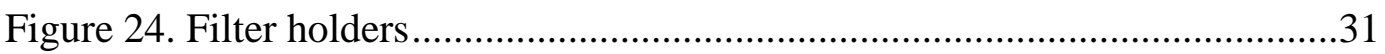

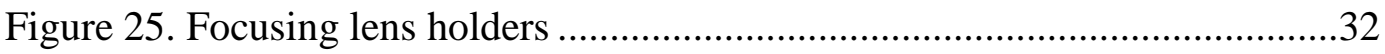

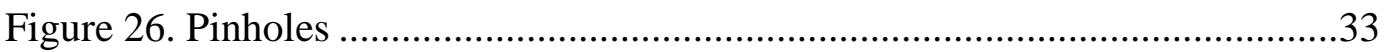

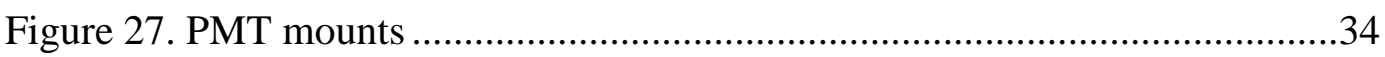

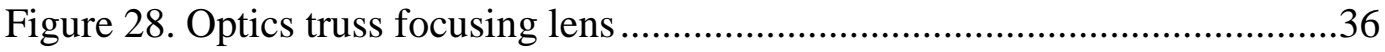

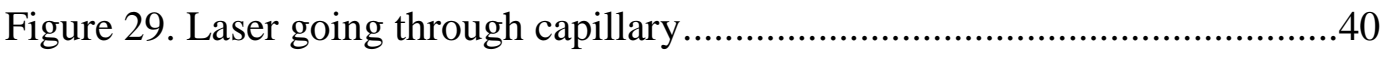

Figure 30. Laser going through capillary and hitting beam blocker .......................41

Figure 31. Forward scatter software data .............................................................43

Figure 32. Screenshots of forward scatter data CV of 3.4\% (left) CV of 5.5\%

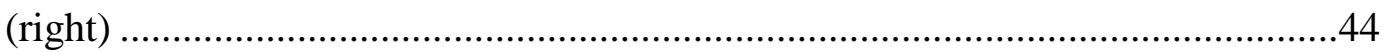

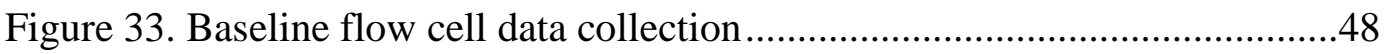

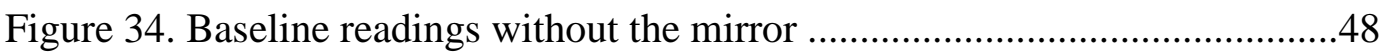

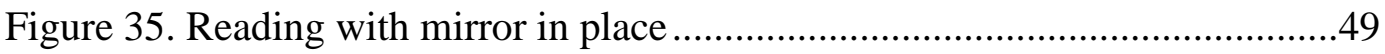

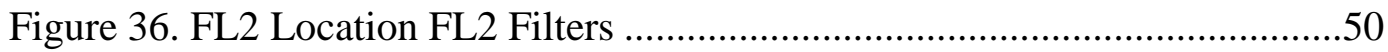


Figure 37. FL1 Location FL1 Filters …....................................................... 50

Figure 38. Baseline FL2 ......................................................................... 51

Figure 39. 8 peak data taken from FL2 on H9307-05 PMT .............................. 53

Figure 40. 8 peak data taken from FL2 on H9305-02 PMT ..............................5 54

Figure 41. 8 peak data taken from FL2 on Yahoo system .................................55

Figure 42. Baseline of optics FSC (left) FL1 (right) ......................................58

Figure 43. New optics FSC (left) FL1 (right) ...............................................58

Figure 44. FL2 with a loosened side scatter focus lens ...................................6 60

Figure 45. FL2 with a tight side scatter focus lens (Screen capture) ..................6 61

Figure 46. Complete assembly of new alignment optics ................................62 62

Figure 47. Beam shaper for collimated laser ............................................... 64

Figure 48. Laser alignment with beam shaper CV 3.94\% (left) vs BSR into

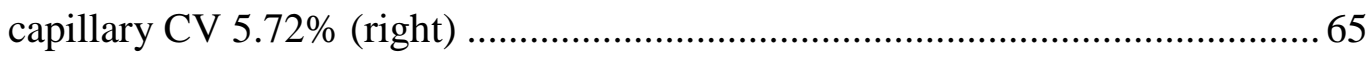

Figure 49. FL2 data resolution with 15mW laser ...........................................6 66

Figure 50. FL2 data resolution with 40mW laser ..........................................67 67

Figure 51. Bluestone data resolution of FSC (left), FL1 (middle), and FL2 (right)

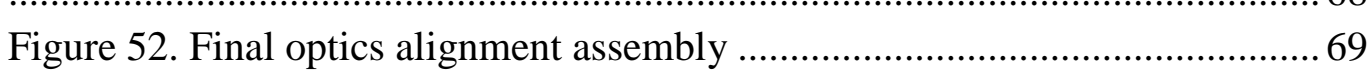

Figure 53. Focal mirror assembly attached to flow cell................................. 70

Figure 54. Zoom of light baffles ............................................................. 71

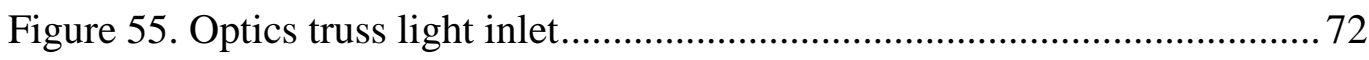

Figure 56. Lid integration with optics truss at light inlet................................ 73

Figure 57. Completed assembly of CytoFlow optics..................................... 74 


\section{Introduction}

Flow cytometry is a tool that can be used to analyze the physical as well as chemical properties of particles. Its aim is to quickly analyze cells using their size and internal complexities. This tool is widely used for analysis of cells in the human body [1]. Some examples of the fields where this tool is applicable as well as the cells it can analyze are seen in Table 1. This table shows the common biological data that is run on a flow cytometer. Looking at these cells can help to diagnose a patient or to observe the progress of a disease that is in the blood. The device is able to tell the user the difference between normal healthy human blood cells or see slight differences in blood cells. 
Table 1. Application for flow cytometry

\begin{tabular}{|c|c|c|}
\hline Field & Clinical application & Common characteristic measured \\
\hline \multirow[t]{4}{*}{ Immunology } & $\begin{array}{l}\text { Histocompatibility cross- } \\
\text { matching }\end{array}$ & $\mathrm{IgG}, \mathrm{IgM}$ \\
\hline & Transplantation rejection & CD3, circulating OKT3 \\
\hline & HLA-B27 detection & HLA-B27 \\
\hline & Immunodeficiency studies & $\mathrm{CD} 4, \mathrm{CD} 8$ \\
\hline \multirow[t]{2}{*}{ Oncology } & $\begin{array}{l}\text { DNA content and } S \text { phase of } \\
\text { tumors }\end{array}$ & DNA \\
\hline & $\begin{array}{l}\text { Measurement of proliferation } \\
\text { markers }\end{array}$ & $\mathrm{Ki}-67, \mathrm{PCNA}^{1}$ \\
\hline \multirow[t]{10}{*}{ Hematology } & $\begin{array}{l}\text { Leukemia and lymphoma } \\
\text { phenotyping }\end{array}$ & Leukocyte surface antigens \\
\hline & $\begin{array}{l}\text { Identification of prognostically } \\
\text { important subgroups }\end{array}$ & $\mathrm{TdT}, \mathrm{MPO}$ \\
\hline & $\begin{array}{l}\text { Hematopoietic progenitor cell } \\
\text { enumeration }\end{array}$ & CD34 \\
\hline & $\begin{array}{l}\text { Diagnosis of systemic } \\
\text { mastocytosis }\end{array}$ & $\mathrm{CD} 25, \mathrm{CD} 69$ \\
\hline & Reticulocyte enumeration & RNA \\
\hline & $\begin{array}{l}\text { Autoimmune and alloimmune } \\
\text { disorders }\end{array}$ & \\
\hline & Anti-platelet antibodies & IgG, IgM \\
\hline & Anti-neutrophil antibodies & IgG \\
\hline & Immune complexes & Complement, IgG \\
\hline & $\begin{array}{l}\text { Feto-maternal hemorrhage } \\
\text { quantification }\end{array}$ & Hemoglobin F, rhesus D \\
\hline \multirow{2}{*}{$\begin{array}{l}\text { Blood } \\
\text { banking }\end{array}$} & Immunohematology & Erythrocyte surface antigens \\
\hline & $\begin{array}{l}\text { Assessment of leukocyte } \\
\text { contamination of blood products }\end{array}$ & $\begin{array}{l}\text { Forward and side scatter, } \\
\text { leukocyte surface antigens }\end{array}$ \\
\hline \multirow[t]{2}{*}{$\begin{array}{l}\text { Genetic } \\
\text { disorders }\end{array}$} & PNH & CD55, CD59 \\
\hline & Leukocyte adhesion deficiency & CD11/CD18 complex \\
\hline
\end{tabular}

Flow cytometry is used all around the world but is such a specialized topic that there is a limited public knowledge of the system. However, it has recently taken off in the past decade. It has become a key piece of research equipment for clinical laboratory work [1]. 
This was caused by the development of smaller, user friendly, less expensive instruments [2]. These devices can be used to look at DNA content analysis, leukemia and lymphoma phenotyping, immunologic monitoring of HIV-infected individuals, and properties of leukocytes and platelets [2]. With the increasing need for smaller, more precise, and customized machines CytoFlow was founded in 2013. CytoFlow is a new contender to the consumer based flow cytometer.

The aim of this project was to introduce a new flow cytometer to market. By working with Cytoflow the goal of this project was to create a new brand of cytometer that could be easily customized to a consumer's needs. Current cytometers are all created the same way by a company. Once the consumer purchases the product it must be modified inhouse to fit their specific need. The final goal of Cytoflow is to create a device that can fit the customers' needs allowing easy implementation for specifications that were designed for the customer purchasing the product. 


\section{Background}

The first application of counting cells while in flow was recorded in 1934 [3]. This machine was known as the Coulter Counter. This machine operated by passing red blood cells through a capillary stage and each cell passing by was counted by a photoelectric apparatus [3]. In 1949 the Coulter Counter was filed for patent [3]. The technology introduced by the Coulter Counter has since been used and refined to produce new technologies. The Coulter Counter led to the introduction to flow cytometry.

The invention of the flow cytometer let to the development of many different advances in cell analyzing. Flow cytometry has been used for studying the cell cycle, apoptosis, cellular and molecular profiles, cellular molecular detection, cell proliferation, transfection, cell viability, and immunology [4]. Recent research has shown that flow cytometry could be invaluable in drug screening and drug development [4]. Flow cytometry was originally produced for hospital use. These devices were often times large and very complex. As time went on a market developed for a cytometer that could be created for personal use as a diagnostic tool for use in portable testing as well as clinical testing. In 2006, BD Accuri C6 introduced the first personal flow cytometer, a new type of machine that was compact and more affordable to the general public [4]. This creation introduced the market of personal flow cytometers that Cytoflow hopes to capture.

Cytometers use a variety of different technologies to create a working system. All components are optimized in order to achieve a working system. The main components consists of microfluidics, electronics, and optics. These three major components are 
interwoven into a cytometer through the six subcomponents of a system. [5] These components include a flow chamber, laser, light detectors, filters, fluidic pumps, and a computer [5]. Before the flow cytometer can be used the cells must first be treated. Using markers cells can be stained with fluorochromes, which effectively mark the cells for flow cytometry. [6] An overview of the components used in a flow cytometer can be seen in Figure 1.

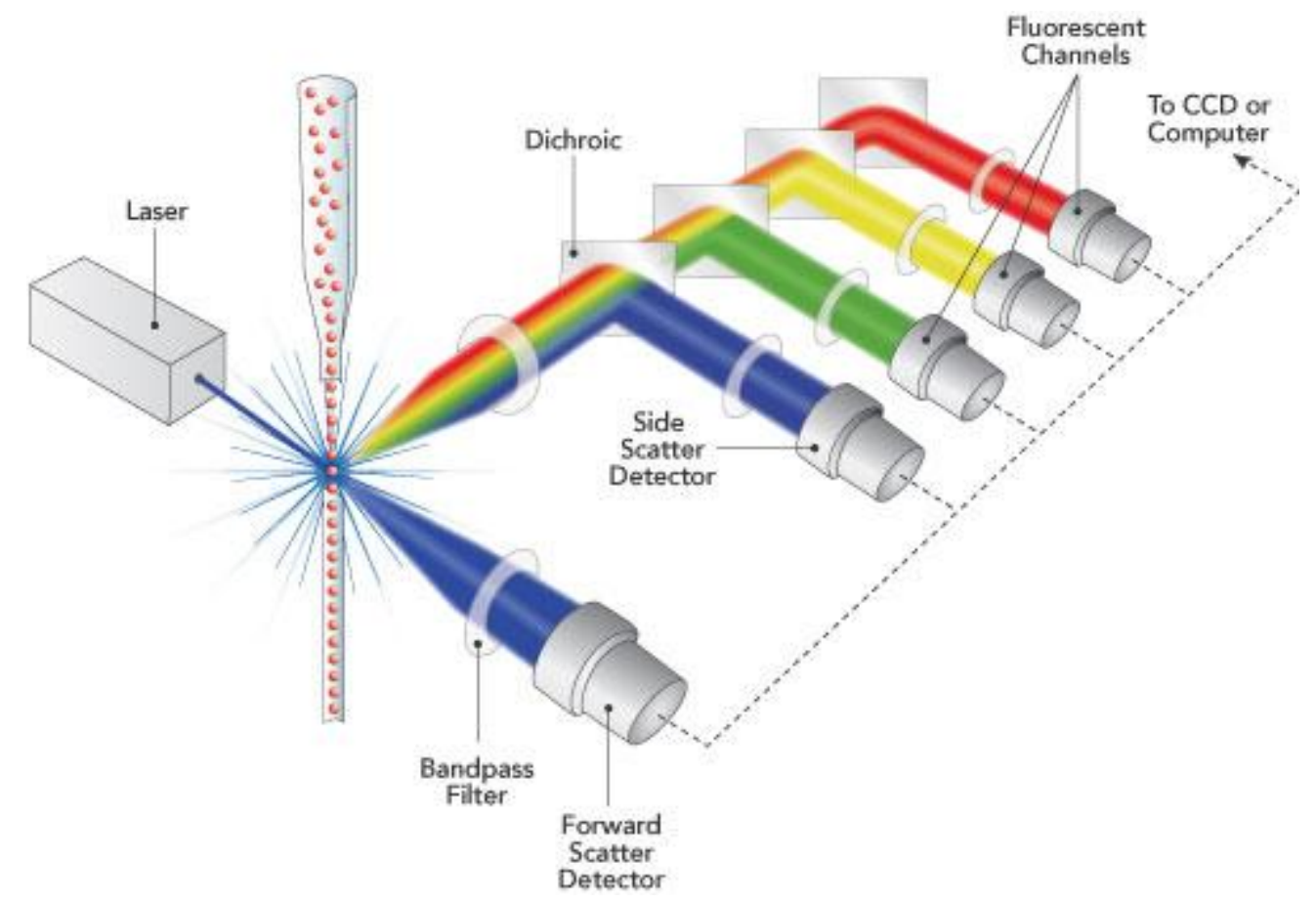

Figure 1. Example of cytometer light path

The flow chamber is responsible for the microfluidic portion of the flow cytometry. The sample cells are run through an optically clear tube. This is usually done by utilizing the laminar flow properties of microfluidics. A fluid often called the sheath fluid is used to help move the sample through the flow cell. The laminar flow properties stop these two fluids from diffusing into one another. This essentially creates what can be referred to as a cylinder concentric to another cylinder. The inner cylinder is the sample with the 
properties that are to be analyzed [8]. The outer cylinder is the sheath fluid with known properties by the observer. The sheath fluid is usually a buffer fluid that will help to suspend and maintain the sample cells [8]. The sheath fluid also helps to align the sample fluid in the middle of the flow cell [8]. A laser is then placed perpendicular to the flow cell and focused on the sample "core" [8]. As the cells pass through the laser they are illuminated by the laser light. The light that hits the cells create different scatters of light [8]. These are broken into forward scatter and side scatter [8].

\section{Light Optics}

There are three major concepts that must be understood to follow the pathway of light in a flow cytometer: light bending, separation, and filtration.

\section{Light Bending/ Focusing}

Light can be manipulated in many different ways using different lenses. Collimated light is light that does not have an angle associated with it. It would travel in a straight line forever like that seen in the image below Figure 2.

Figure 2. Image of collimated light 
The above image portrays light that is traveling out of a collimated source. The light will continue to travel in a straight line unless acted upon by an outside factor. In a 3D dimension this light can be thought of as a column of light whose properties do not change.

However, if collimated light passes through a convex lens it would focus down to a point equal to that lenses focal point much like that seen in Figure 3 below.

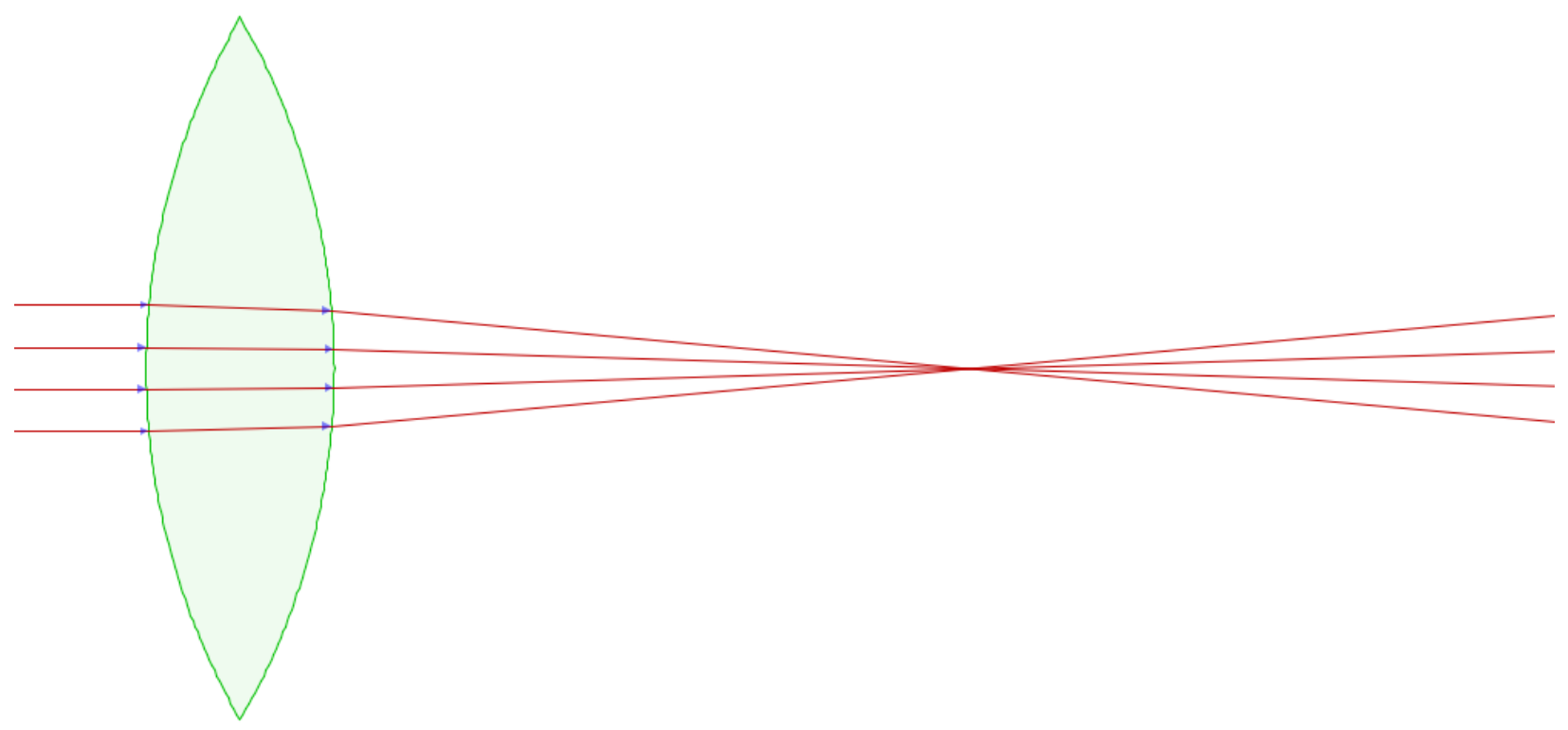

Figure 3. Image of light focused via a lens

Light that passes through the lens will be focused into a single spot known as the focal point. It is here that the light will be at its greatest intensity. The properties of the lens the light passes through will determine how close or far away the focal point is from the lens. From the focal point, light will continue to travel in the direction it was heading unless changed. From here light can be collimated again if needed. If another lens is placed its focal distance away from the focal point of light, the light can become 
collimated again causing the light to travel again with no associated angle see Figure 4 below.

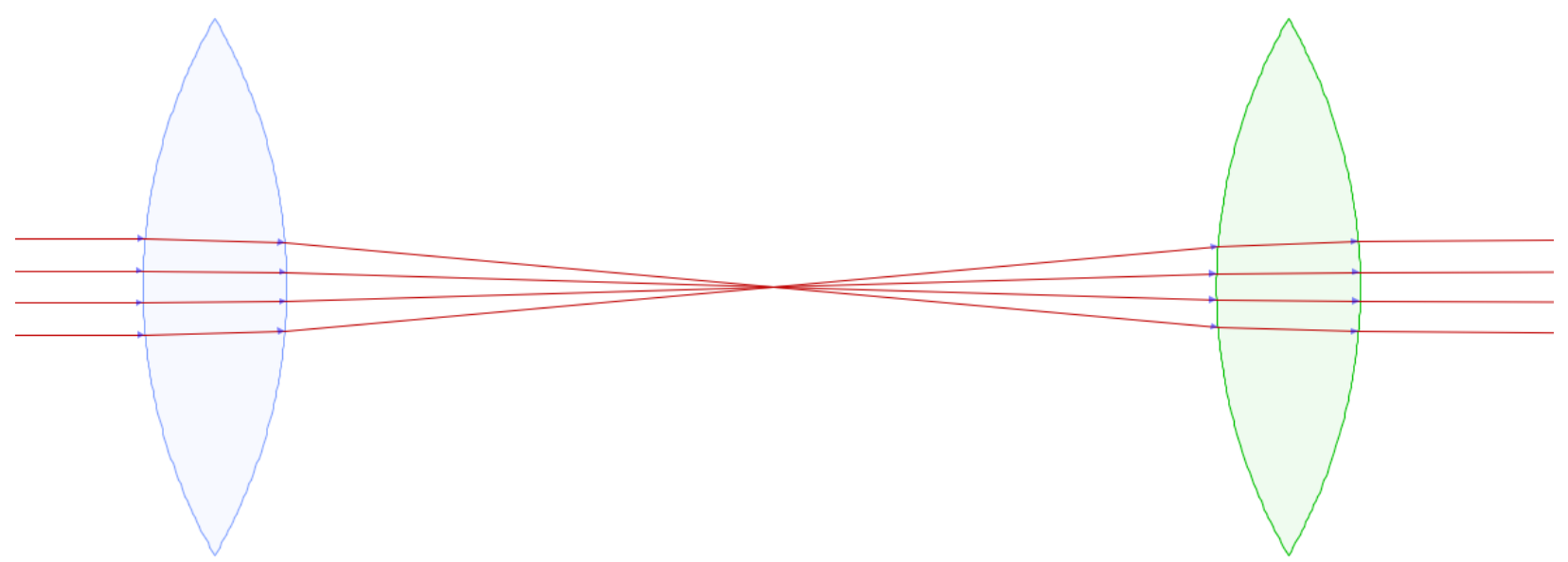

Figure 4. Light being focused then collimated again

If required the focal depth of light in the $\mathrm{x}$ and $\mathrm{y}$ direction can be achieved independently. This would often be done if specific properties are required for light. Creating this type of light focusing can be done using plano convex lenses. A plano convex lens has a profile that looks like a "D" that is to say it will only focus light along one plane. Depending on the orientation of the lens it can either focus light in the $\mathrm{x}$ or $\mathrm{y}$ direction. If two plano convex lenses are used in conjunction with each other they can focus a collimated beam along the $\mathrm{x}$ and $\mathrm{y}$ axis at two different points depending on their focal length. The reason why this is necessary will be explained in greater detail under the Laser design section.

\section{Separation/Filtration}

Separation of light is used in a flow cytometer to analyze the desired wavelengths of light. This is usually completed by using dichroic mirrors. A dichroic mirror is a mirror 
with significantly different reflection and transmission properties for varying wavelengths [9]. This means that at certain wavelengths it will act as a mirror reflecting the light and at other wavelengths it will allow light to pass through it. These come in two varieties, long-pass and short-pass. A short-pass dichroic mirror will transmit any wavelength of light that is below the cutoff of the mirror and reflect any wavelength above the cutoff [9]. For instance a short-pass dichroic mirror that is $450 \mathrm{~nm}$ will allow wavelengths of light less than $450 \mathrm{~nm}$ pass through it and reflect wavelengths of light above 450nm. A long-pass dichroic mirror works in the opposite way of the short-pass mirror. It will transmit any wavelength of light that is above the cutoff of the mirror and reflect any wavelength of light below the cutoff [9]. When further filtration is needed a notch filter can be added in conjunction with a dichroic mirror. A notch filter is the equivalent of a short-pass and long-pass dichroic mirror combined [8]. The filter has two cutoff values and can work in two different ways. The filter can either transmit the wavelengths between the two cutoff values and reflect the wavelengths outside of the values or work in the opposite way.

\section{Scattered Light}

When the laser hits the cells passing through the flow cell it causes laser light to be scattered. There are two main types of light that are collected in cytometry they are known as forward scatter and side scatter [10]. This information is used to distinguish between cells and types creating populations of similar cells. The forward scatter light correlates to the cell size whereas the side scatter depends on the density of the particle [10]. An example of what this scatter looks like can be seen in the image below Figure 5. 


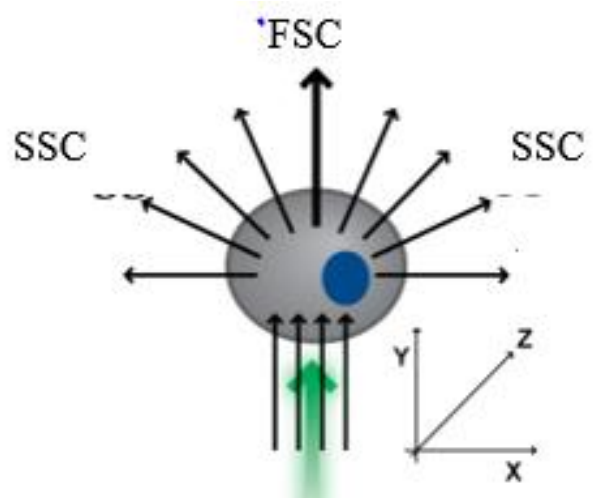

Figure 5. Light Scatter off a cell [10]

The forward scatter from the cell, abbreviated FSC, is on the same axis as the laser while side scatter, abbreviated SSC, is that produced at all angles relative to the laser [10]. For the particular flow cell used in this project, only the side scatter that is orthogonal to the laser is collected. This can be seen when looking at the images of the flow cell. This reading becomes particularly important when analyzing blood cells, as a comparison between forward scatter and side scatter creates different populations of cells [10]. For instance a large granular cell would produce a high forward scatter and high side scatter, while a large, not so granular cell would produce high forward scatter with low side scatter [10]. Below in Figure 6 is an image that shows how the populations are separately analyzed using forward and side scatter. 


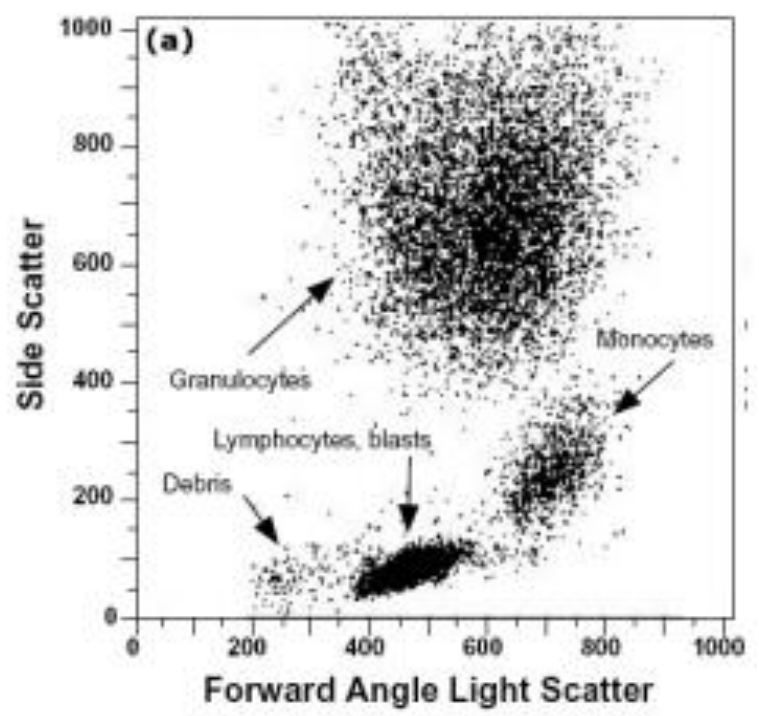

Figure 6. Example image collected from whole blood [10]

The image shows populations of different cells as labeled in the image. This information can be used to see if cells are missing from a sample or look for markers placed in the sample by the user. The side scatter light that is collected is then used to produce fluorescent data. Using a combination of dichroic mirrors and filters different wavelengths of light can be channeled into detectors. The wavelength of light that reaches the detectors will be determined by the mirrors and filters in the system. The photons that enter the detector will then cause a voltage to occur and this is recorded by the software. When the light hits the cells the fluorochromes they contain will emit a certain wavelength known by the user. Special optics filters are used to accept and reflect these wavelengths. By analyzing the light that is collected through each filter the user will get a count of the fluorochromes passing through the flow chamber. This will give an accurate count of the cells that pass through the flow chamber. [5] Once this data is collected it can be analyzed in computer software, which allows the user to look at the 
size of different cells that are marked with the corresponding fluorochrome [5]. The cell data is placed into histogram plots like the image below in Figure 7.

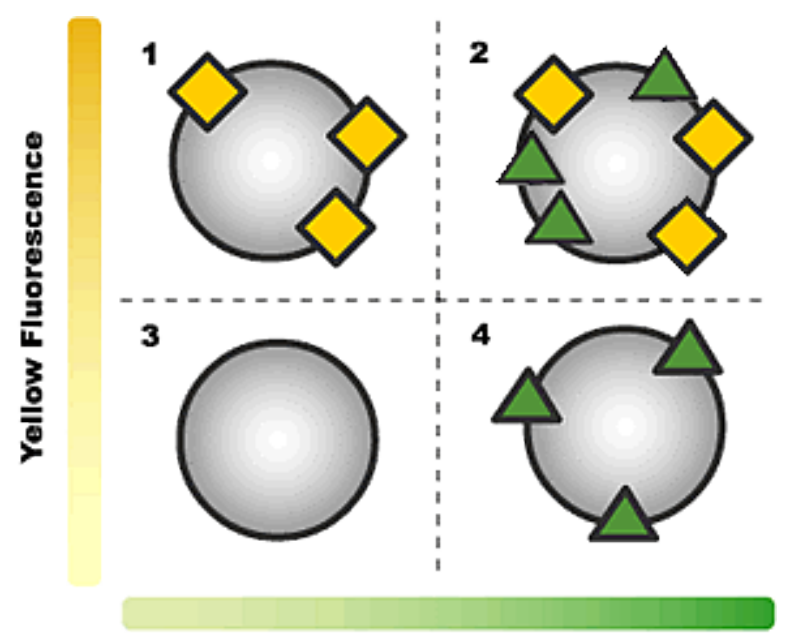

Green Fluorescence

Figure 7. Example of how cells are displaced into regions [5]

This image displays how a bivariate plot of a green fluorescence and yellow fluorescence detector would interact. The $\mathrm{X}$ and $\mathrm{Y}$ axis act as counts for the cell. A cell with no markers on it would have no counts of green or yellow fluorescence causing it to appear in the bottom left corner. While a cell with both markers would appear in the upper right corner. If a cell has only green markers in it, it will stay along the $\mathrm{X}$ axis but vary in count depending how many markers are present. When hit with a laser markers will fluoresce. The color will reach the respective detector and be plotted according to fluorescence intensity. Quadrants can then be looked at when comparing the two color markers. As seen in the image, each combination of markers will appear in their specified quadrants. Data for cells with only yellow fluorochromes will appear in quadrant 1. Data for cells with both green and yellow fluorochromes will appear in 
quadrant 2. Data with no fluorochromes will appear in quadrant 3. Data with only green fluorochromes will appear in quadrant 4.

\section{Photomultiplier Tube}

In order to detect the light that cells produce in a flow cytometer a photomultiplier tube is used. A photomultiplier tube also known as a PMT is a photosensitive device with extremely high sensitivity and response time [11]. The inner working of a PMT can be seen in Figure 8.

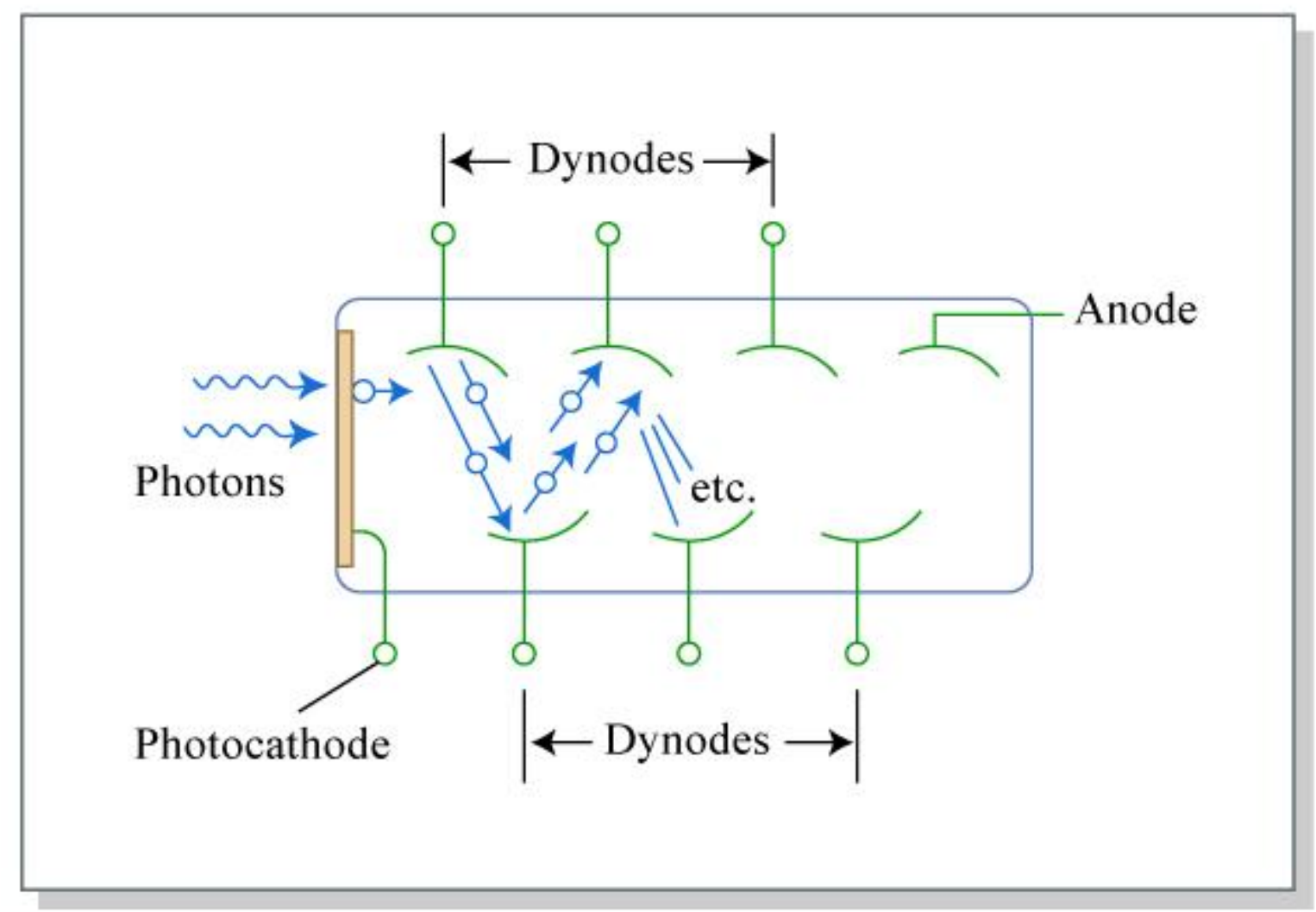

Figure 8. Inside of a Photomultiplier Tube [12]

PMT's are used to capture photons and output a signal based on this premises. A PMT works by light entering through a glass window and exciting electrons that are passed 
through the device by its power cable. Depending on the intensity of the photon a number of electrons will be excited in the device [11]. The electrons will then travel through the device and be amplified during travel by bouncing off the dynodes [11]. The electrons are eventually collected and produce a voltage to be passed out of the PMT. From here the signal is passed through a coaxial cable for examination. In some applications the PMT can be paired with a preamplifier to further enhance the signal given from the PMT.

A preamplifier, also known as a preamp, is a device that takes in a signal as an input and amplifies that signal and passes it as an output. The preamp in this field will be taking the voltages produced by the PMT and amplifying it before passing it on for data processing. 


\section{Objective}

The goal of flow cytometry is for the user to be able to quickly diagnose diseases that have an effect on cell shape and size. The reason flow cytometry has become an up and coming development is because of the advantages that can come with this system. A flow cytometer is able to process a tremendous amount of data in a short amount of time. By adjusting the microfluidics of the system the user is able to collect up to 500 data points per second. This allows the user to run a significant amount of data and use statistical analysis to determine differences in cells. Some cytometers are able to process cells and direct them to a specified bin according to fluorochromes of the cell [13]. The cells can then go through further analysis if needed. Currently there are still problems and limitations with flow cytometry. One of the first issues is the cost accompanied with a cytometer. The cytometry machines can sometimes run upwards of $\$ 75,000$ [13]. These machines also require a very qualified and trained technician. [13] Flow cytometry is also limited by the requirements necessary to run a sample. Cells must be run through the cytometer suspended in a fluid. This means that intact tissue architecture cannot be analyzed in this method [13]. Cells that have similar fluorochrome expression can be difficult to differentiate between. With current systems the high amount of data that pass through the cytometer can make analysis complex.

Cytoflow hopes to create a customer based cytometer. This means that all aspects of the machine will be customizable. A customer will be able to specify the wavelength of the laser that they want to use to analyze the cells. They will also be able to choose the wavelength of the fluorochromes they would like to look at. This will create a complete 
system that is created to meet the customer needs for their work. These customers could be trying to analyze anything from the progression of AIDS in the blood to a reagent that is added to an oil sample. This tool would be viable for looking at samples that need to be run at a high throughput in a small amount of time.

The objective of this research is to help optimize the current Cytoflow cytometer. The aspect reviewed in this document will focus on the optical setup of the system. This will include the following components: laser, flow cell, forward scatter, and fluorescence signal. This research should look into the possible and so far unexplained events causing distortion of optical data. Improvements will be based on comparisons of Spherotech Rainbow Calibration Particles. Calibration beads have a known size and a known particle composition. The beads are expected to give off certain values in all detectors in a cytometer. Research should help to resolve all data that is expected when using calibrated bead testing. Cytoflow would like to hit the market with data that is comparable to the top cytometers currently being produced. This is done by comparing density plots of human blood to plots made by a preexisting device. This can be done by matching cell populations that show up in the blood. If a cell is a different size or has a different internal structure it will show up in a different position on the density plot. 


\section{Design}

The optical pathway of CytoFlow's cytometer consists of six major components in the assembly. The flow cell, forward scatter block, and optics truss were designed in house and SLM 3D printed by a third party. The other three components will be OEM parts which are the PMTs, optics glass, and the laser. Below is the starting iteration of the design work to create the CytoFlow cytometer. As seen in Figure 9 the first iteration of the design was created.

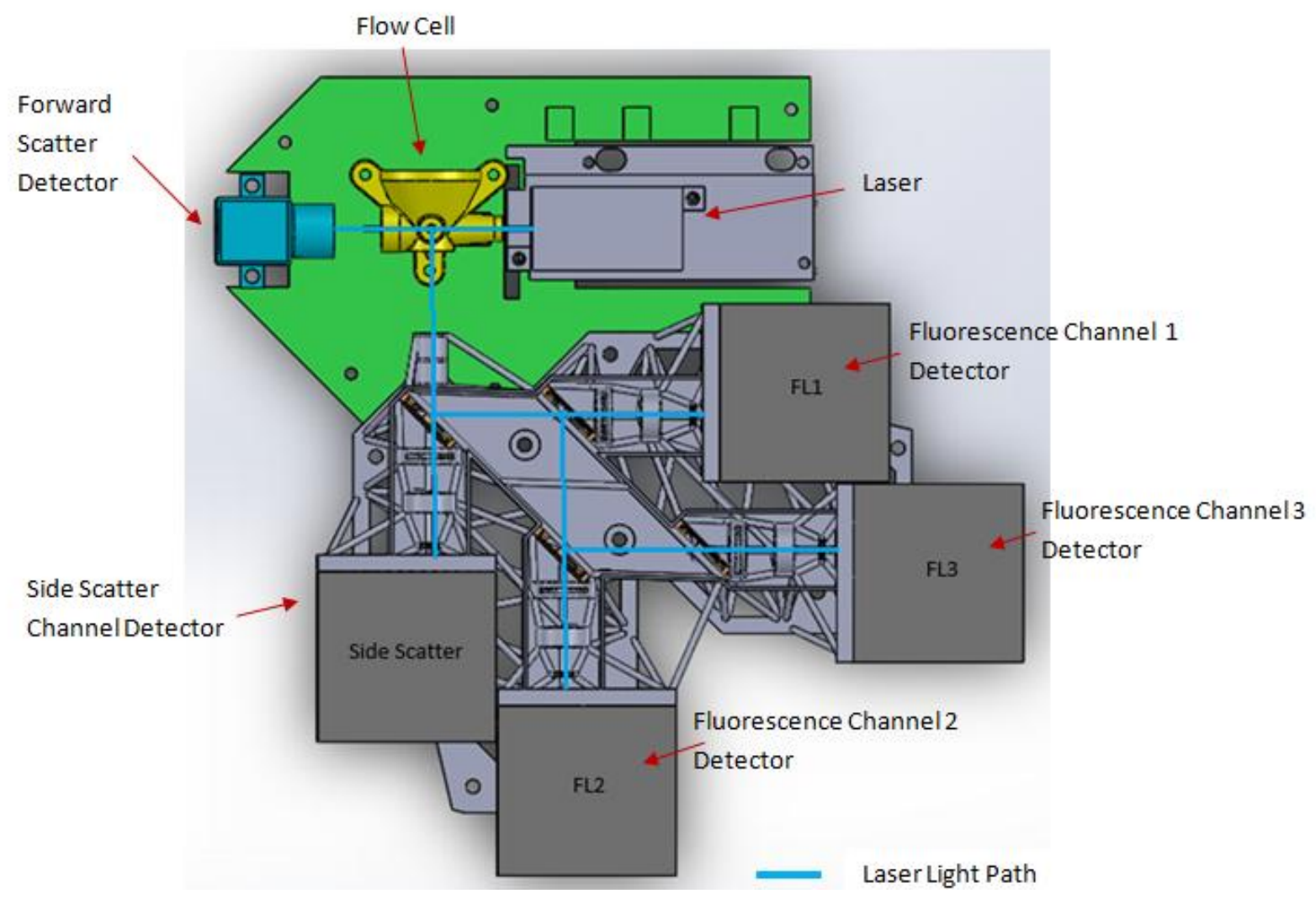

Figure 9. First iteration design with light path in blue

The light path comes out of the laser at a height of $19 \mathrm{~mm}$. It then travels through the flow cell hitting the capillary which the sample runs through. The cells are illuminated 
with light causing forward scatter and side scatter. These are picked up by the labeled detectors. From there the light is passed along to the other channels and detectors using a combination of dichroic mirrors. The wavelength of light that travels through each channel is determined by the dichroic mirrors that are used to in front of each channel path. The parts that make up the completed assembly will now be looked at in greater detail.

\section{Flow Cell}

Before the cytometer could be created a flow chamber first had to be fashioned. This part was created using SolidWorks and printed using a SLM 3D printer. The image of the flow cell for this project can be seen below in Figure 10 and Figure 11.

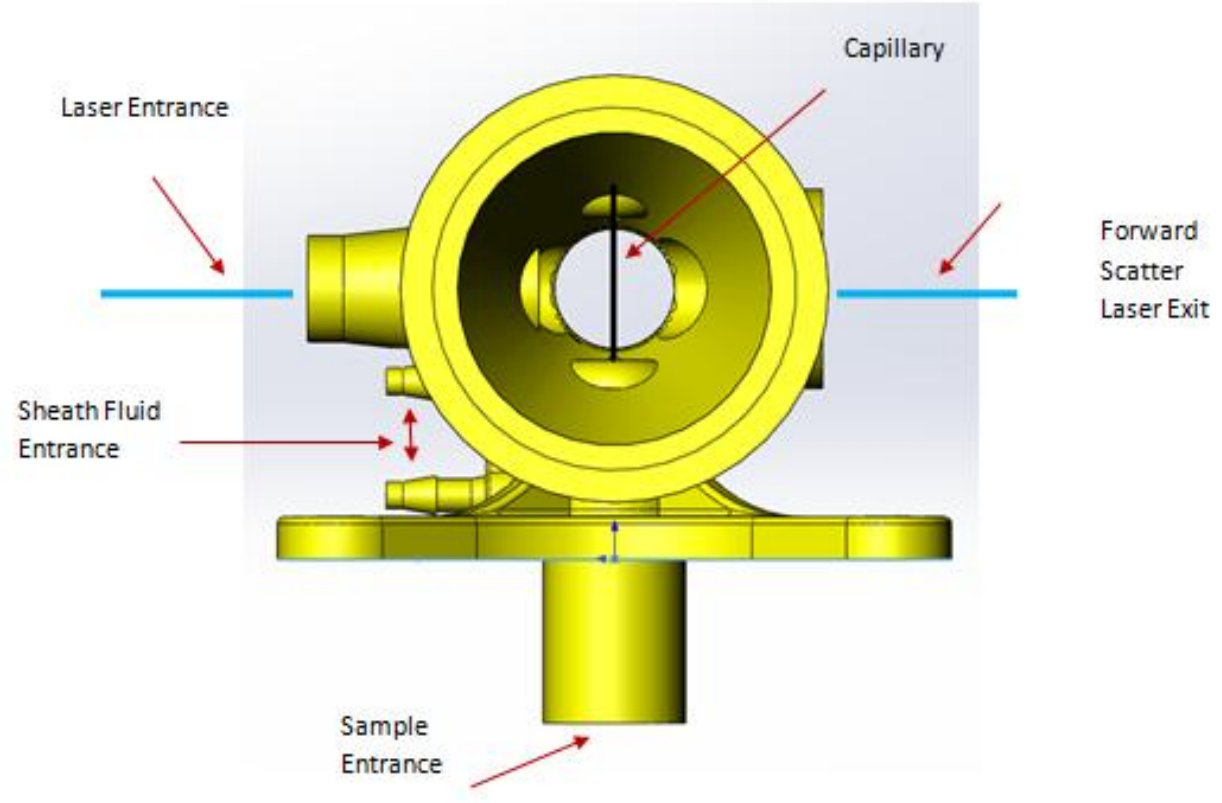

Figure 10. Front view of flow cell 


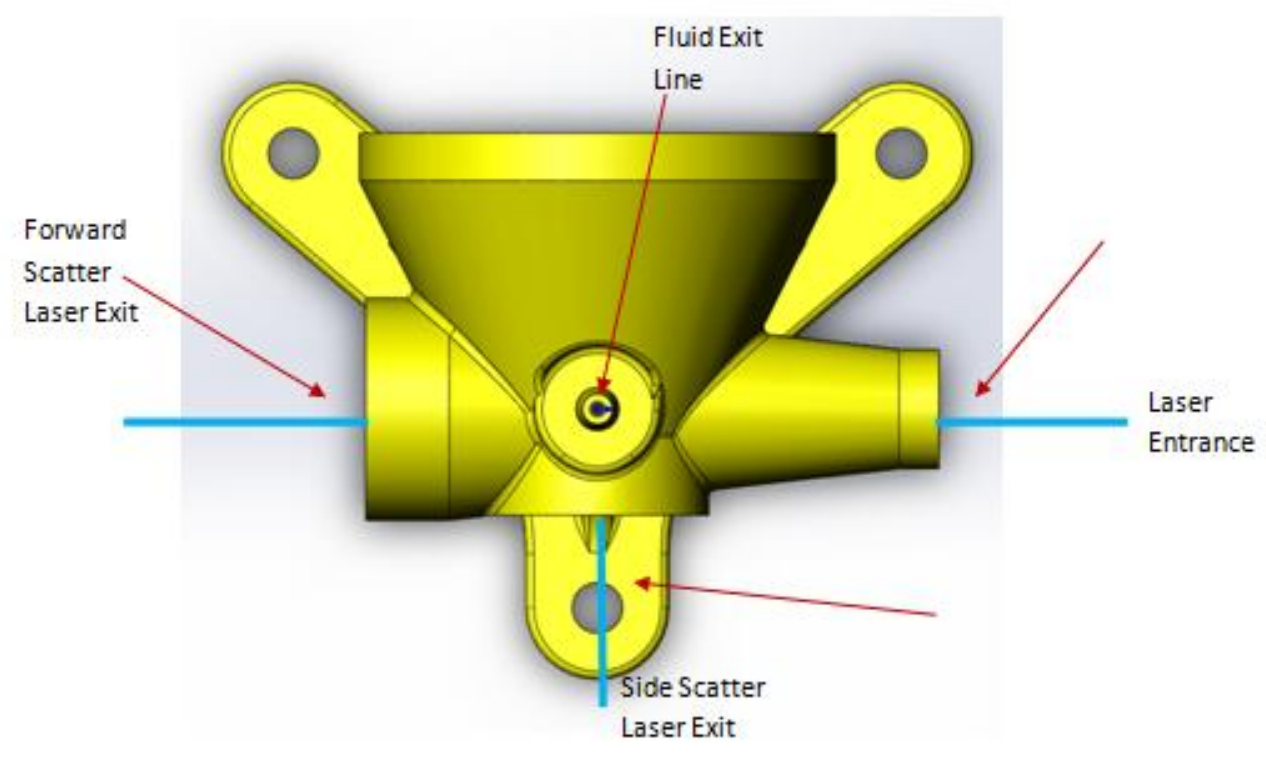

Figure 11. Top view of flow cell

The center of the flow cell stands at $19 \mathrm{~mm}$ tall. This was done because most standard issued lasers have a beam exit of $19 \mathrm{~mm}$. This would allow for the flow cell and a standard laser to be placed into the system without any special mounting. As stated earlier the laser will enter the flow cell at the entrance. From here it will hit the sample that is traveling up through the capillary. The laser will exit via the forward scatter and side scatter exits.

\section{Laser}

In order for the laser to appropriately hit the capillary in the flow cell specific laser properties were required. The beam had to have different focal lengths in the $\mathrm{X}$ and $\mathrm{Y}$ axis. The laser was created to produce a Gaussian curve in the X plane. Much like that seen in Figure 12. 


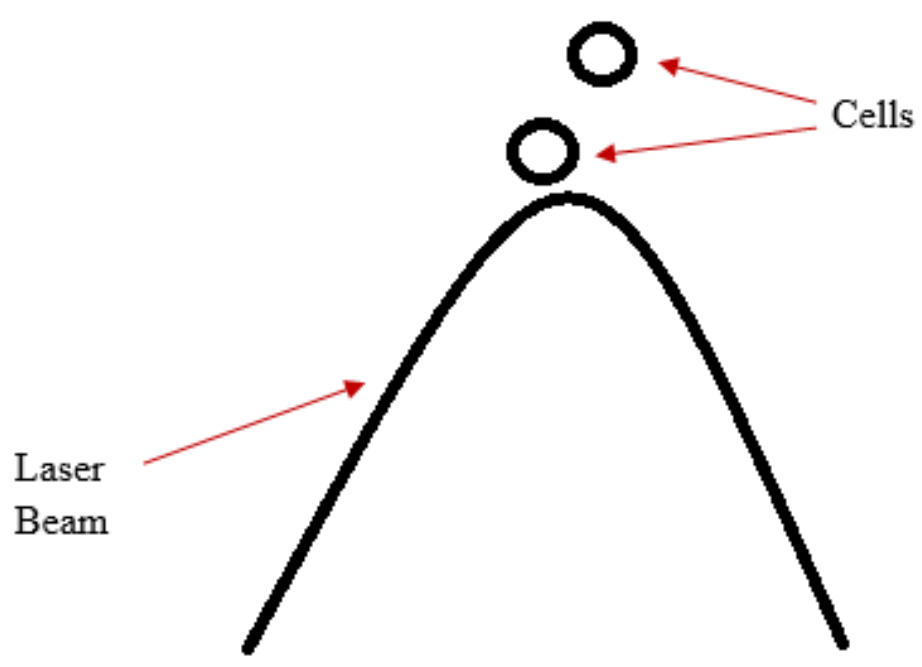

Figure 12. Gaussian curve of beam in $X$ plane

This profile ensured that the entire width of the sample core was illuminated by the laser. That way if a cell did not fall perfectly in line with the other but was still within the core it would receive the full intensity of the laser. In the Y plane the laser was focused in at the capillary. By focusing the laser at the capillary in the $\mathrm{Y}$ axis it ensured that only the time of flight of one cell was analyzed at a time. This is displayed in Figure 13 below.

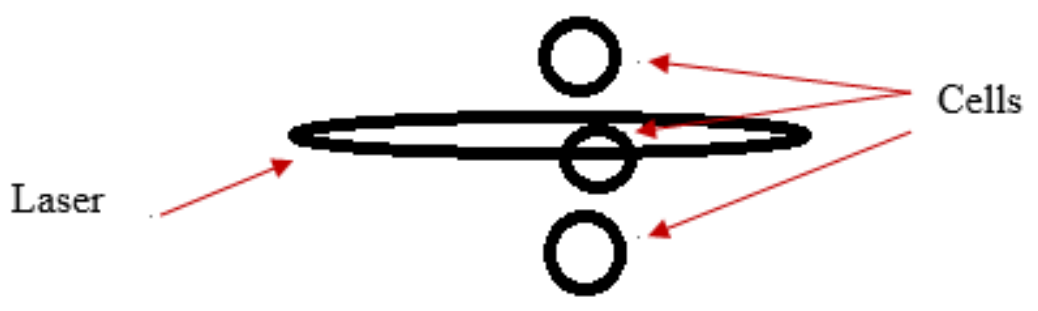

Figure 13. Beam in $Y$ plane with cells passing through

To think of it in another way the laser in the $\mathrm{Y}$ axis was focused tight enough that it could go through the small gaps between cells. This allowed the system to know when it was 
hitting a cell and when it was hitting nothing. If the beam was to large in the $\mathrm{Y}$ axis it would have the potential to hit two cells at once and this would create a false reading. For these reasons the laser had properties of the following. The laser was focused on the capillary in the $\mathrm{Y}$ direction, but focused before the capillary in the $\mathrm{X}$ direction. A laser company called Blue Sky Research (BSR) was contracted to create such a laser for the needs of CytoFlow. The laser profile they created can be seen below in image Figure 14 .

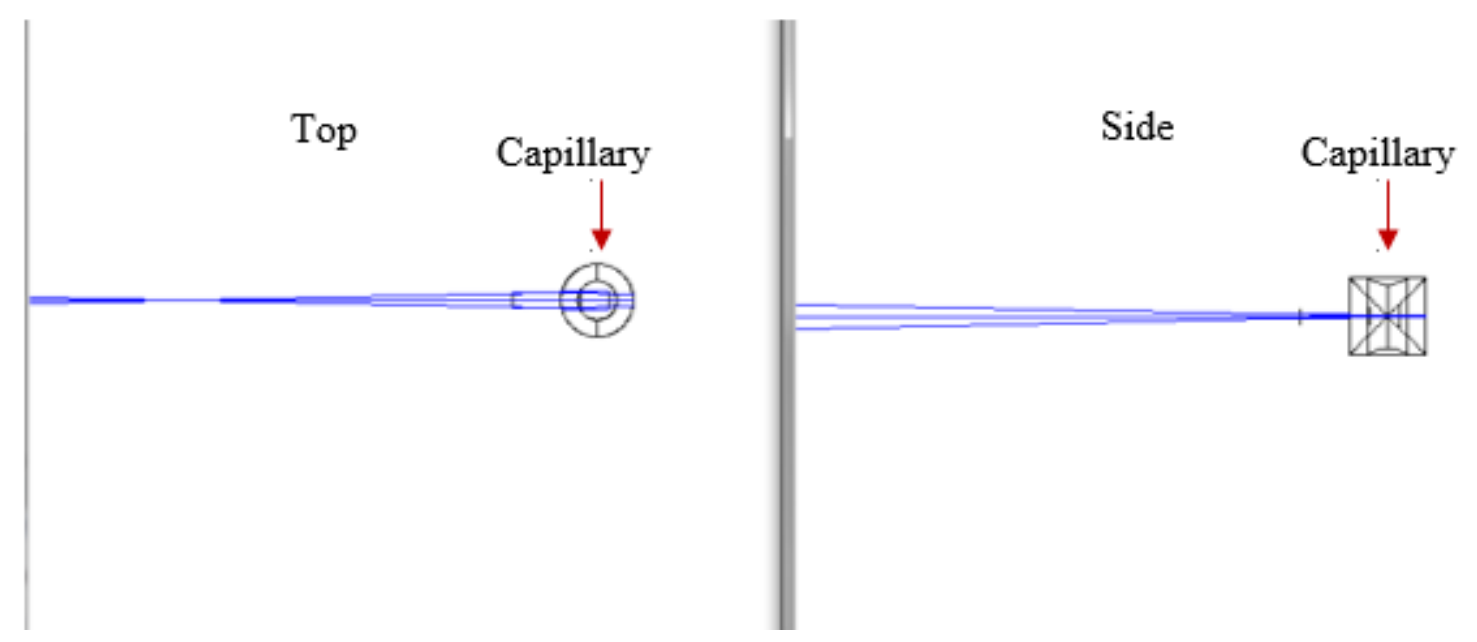

Figure 14. BSR Laser projection from top and side view

In the image above the $\mathrm{X}$ axis focus can be seen on the left and the $\mathrm{Y}$ axis focus can be seen on the right. The reasoning for focusing the beam early in the $\mathrm{X}$ direction is to illuminate the entire capillary in the $\mathrm{X}$ axis as discussed earlier. To create this type of focus Blue Sky Research used two cylindrical lenses mounted inside the laser head. If the beam was to large in the $\mathrm{Y}$ direction the laser light could be in contact with two cells at one time this would give false readings. Having a tight focus in the $\mathrm{Y}$ direction allows the detectors to distinguish where one cell ends and another begins. The readings given 
by Blue Sky Research said that at a laser distance 30mm from the capillary the size of the beam in the $\mathrm{X}$ direction will be $64 \mathrm{um}$ and $17.2 \mathrm{um}$ in the $\mathrm{Y}$ direction. Adjusting the face of the laser to be $31 \mathrm{~mm}$ would change the beam shape at the capillary to the image Figure 15.

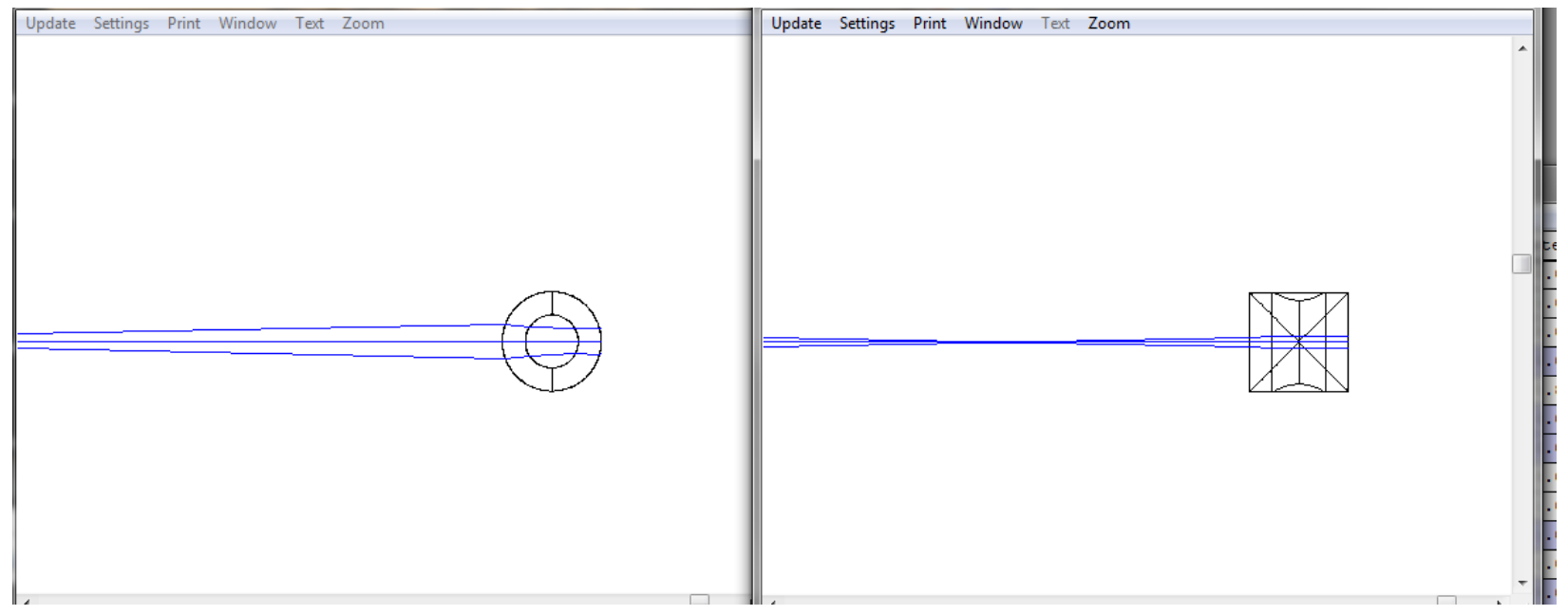

Figure 15. BSR Laser projection at $31 \mathrm{~mm}$ from capillary top view (left) and side view (right)

As shone if the laser was at $31 \mathrm{~mm}$ it would cause the laser to deform in the $\mathrm{X}$ axis as seen by the image on the left. It would also cause the laser to focus before the capillary in the Y axis making for a larger laser that could give incorrect results. This caused a beam shape of 94um in the $\mathrm{X}$ direction and $42 \mathrm{um}$ in the $\mathrm{Y}$ direction. While adjusting the face of the laser to be $29 \mathrm{~mm}$ would change the beam shape at the capillary the opposite direction as seen in Figure 16. 


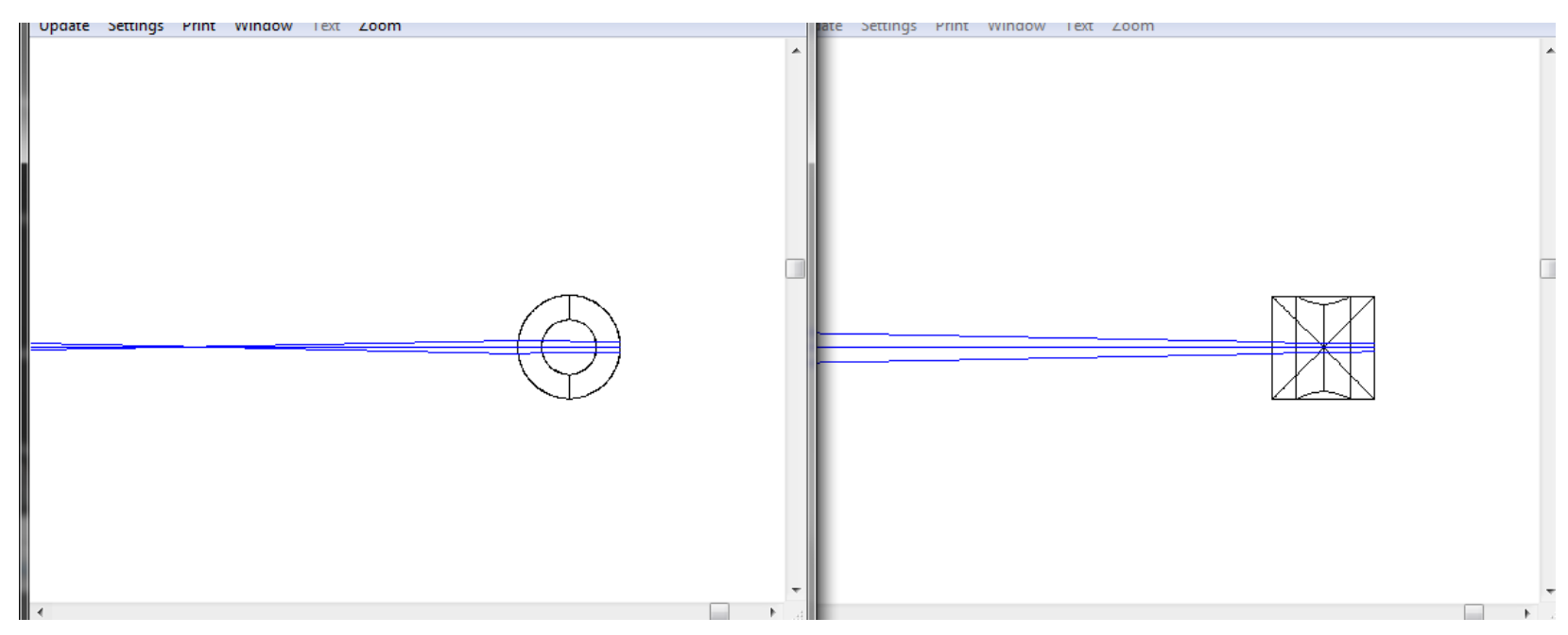

Figure 16. BSR Laser projection at 29mm from capillary top view (left) and side view (right)

Above is the opposite adjustment of the laser positioned at $31 \mathrm{~mm}$ from the capillary. Here the laser focuses to close to the capillary in the $\mathrm{X}$ axis. This causes the beam to be much narrower and gives the chance for a partial illumination of a cell. If the laser catches only the outer edge of the cell the intensity would be off yielding incorrect results. In the $\mathrm{Y}$ direction is much the same scenario of a laser at $31 \mathrm{~mm}$. The $\mathrm{Y}$ axis of the laser would be to large giving the chance of illuminating more than one cell at once.

A positioning of $29 \mathrm{~mm}$ from the capillary caused a beam shape of $46 \mathrm{um}$ in the $\mathrm{X}$ direction and 38um in the Y direction. The laser was very sensitive in its relation to the capillary. The laser beam exit also stood about $6 \mathrm{~mm}$ tall so a special mount had to be created to place the beam in line with the flow cell.

Once the laser was added the resulting assembly looked like that of Figure 17. 


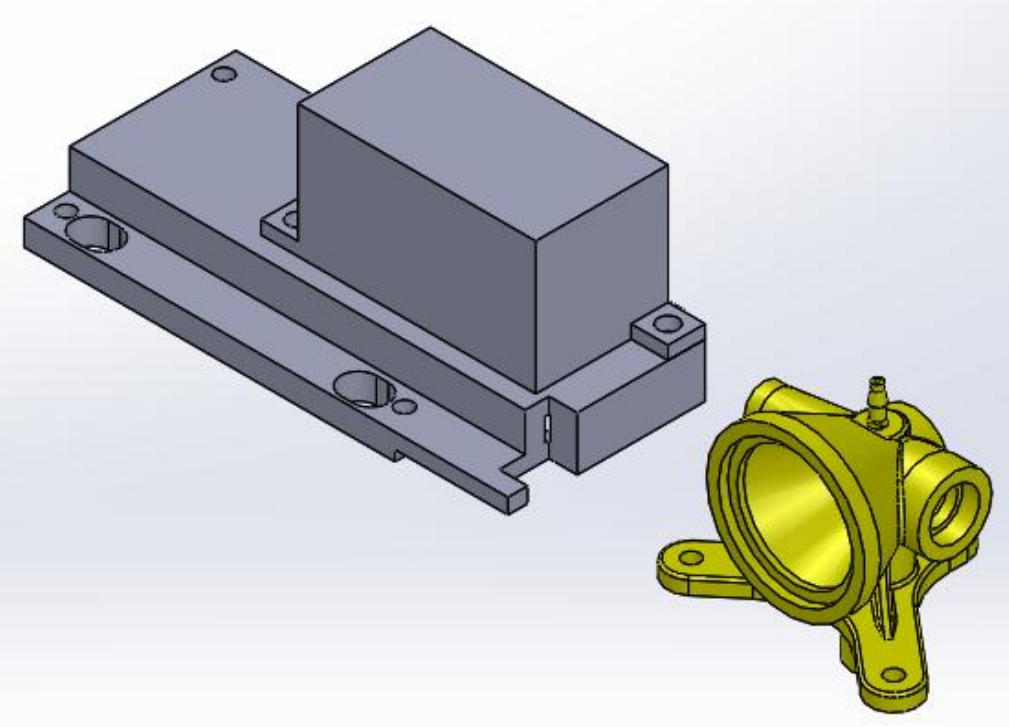

Figure 17. BSR Laser assembly with flow cell

Once the BSR laser was placed onto its mounting assembly this placed the laser at the $19 \mathrm{~mm}$ height needed for correct assembly height. The mount allowed for easy adjustment of the mount in the case that the laser needed to be moved in the $\mathrm{X}$ and $\mathrm{Z}$ planes. This mount also allowed for other integrations of lasers if needed.

\section{Forward Scatter}

Placed inside the flow cell at the forward exit is a focusing lens. This lens is used to help collimate the light that comes off the cells in the capillary. The forward scatter lens is a double-convex lens purchased from Edmund Optics. It has an effective focal length of $9.00 \mathrm{~mm}$. Further specs can be seen in A. The beam is focused and would hit the capillary at the focal point discussed earlier. Then travel onto the detector. When the 
cells were hit by the laser it would look like

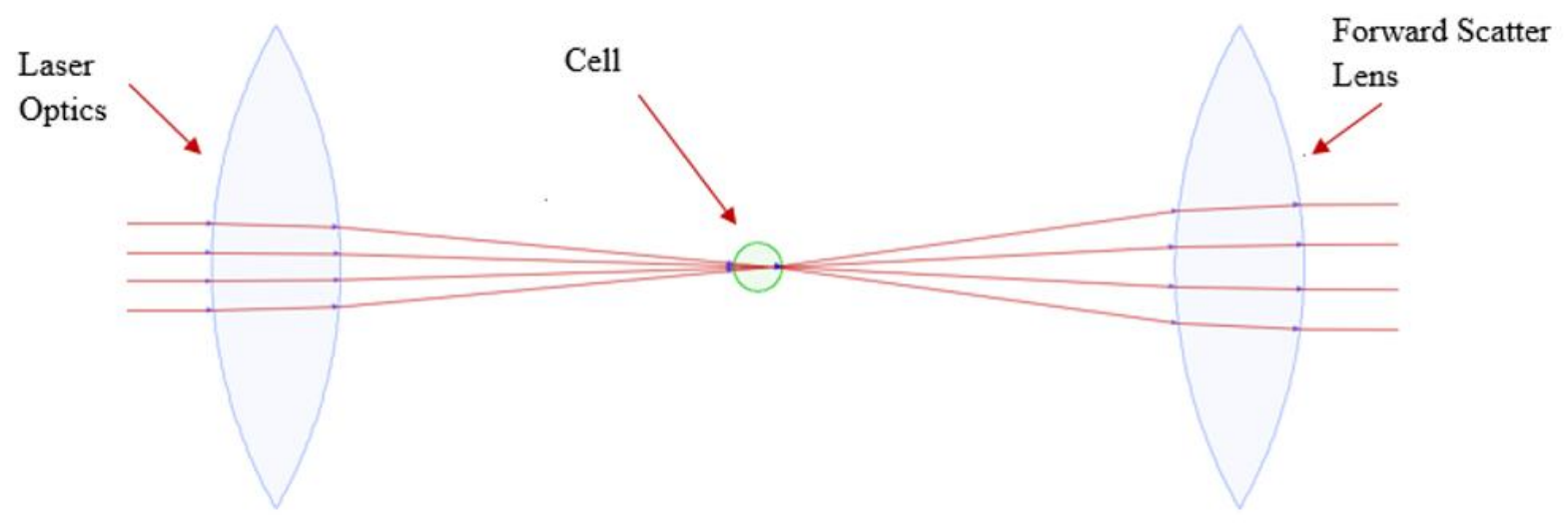

Figure 18.

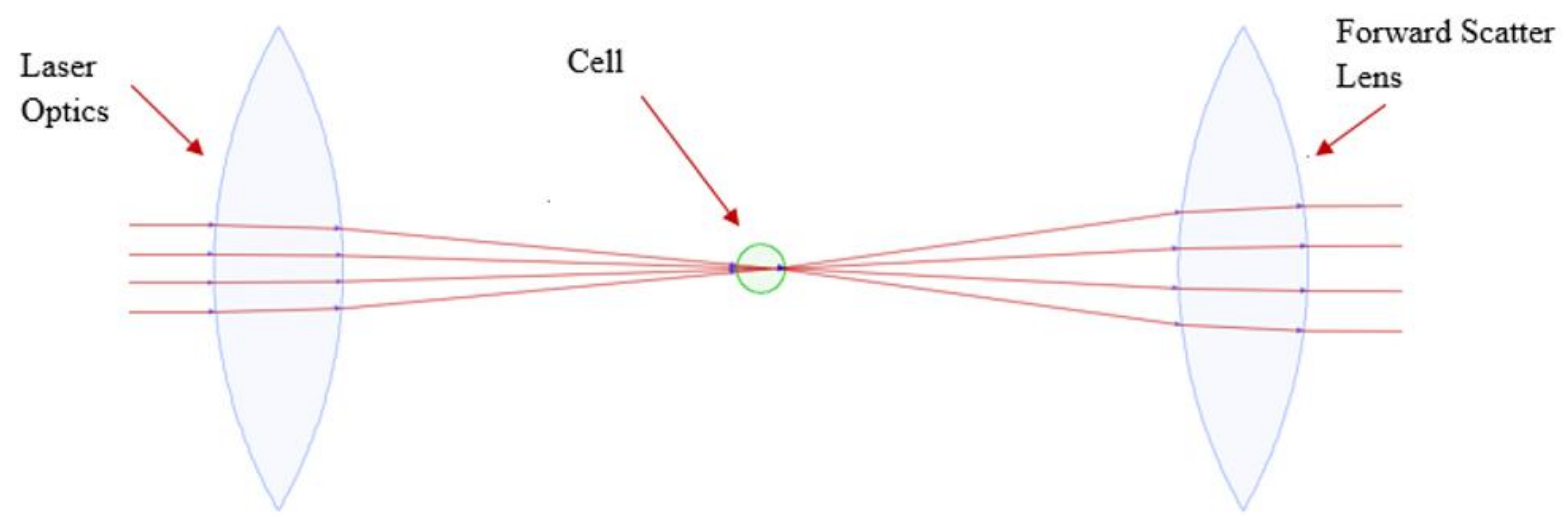

Figure 18. Beam shape passing through a cell

The laser traveling through the cell above causes the properties and direction of the light to change. This change is known as deflection. The forward scatter diode aims to detect this light.

However, with the beam fully entering the forward scatter diode signal, the slight variation of light caused by the cell cannot be seen. The detector would be oversaturated with light and the small deflection of light caused by the cells would be indistinguishable. 
This problem was combated by implementing a cylinder to effectively block the concentrated light of the laser but allow the light refracted off the cell to pass by and reach the detector. Figure 19 shows how this beam blocker would function.

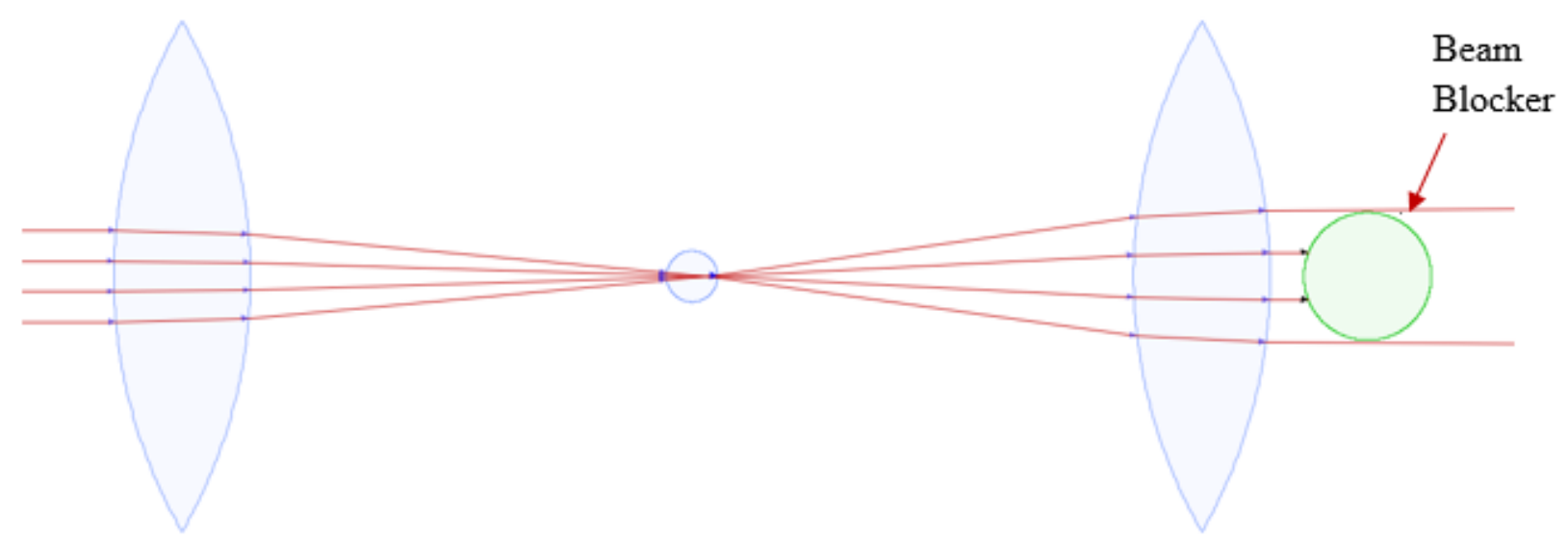

Figure 19. Beam shape passing through cell with beam blocker incorporated

The cylinder or green circle in this image is used to block the concentrated, middle rays, in this image. This allows the faint deflected light to be distinguishable from the noise or background light created by the laser. When a cell is not passing through the light no deflection would occur. This would cause the full intensity of the beam to hit the beam blocker.

A block was created to hold the forward scatter detector as well as the beam blocker. The component can be seen in Figure 20. 


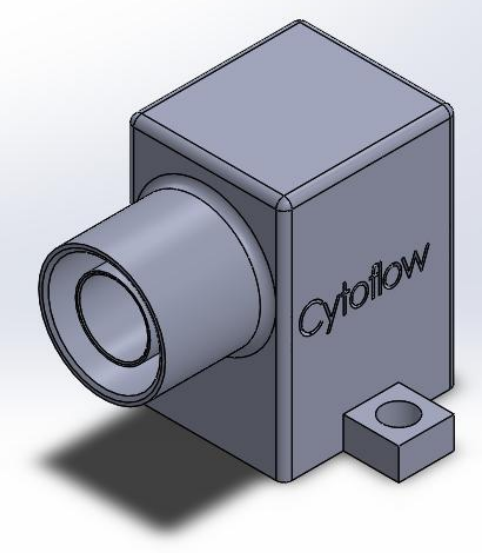

Figure 20. Forward scatter block

This forward scatter block had a cylinder that could be inserted into it. This acted as the beam blocker. The long cylinder protruding from the block was used to encapsulate the flow cell laser exit. The aim of this was to reduce the amount of environmental light that could enter the forward scatter block. The forward scatter block was then added to the assembly as seen in Figure 21.

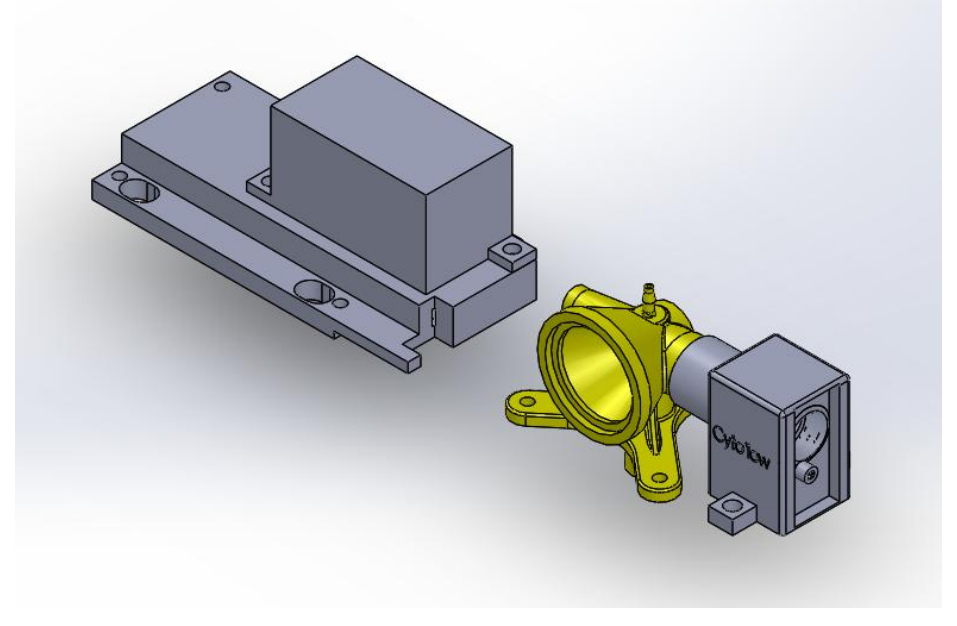

Figure 21. Forward scatter assembly 
This assembly shows the necessities that were needed to complete the forward scatter aspect of the project. Light that was projected from the laser hit the capillary in the flow cell and was collected into the forward scatter block.

\section{Side Scatter}

Next the assembly for the side scatter was created. A lens was placed into the flow cell at the side scatter exit. The side scatter lens would be used to best collimate the beam. This lens was threaded into the flow cell to allow for minor adjustments of depth in relation to the capillary. The focal length of the lens was $3.1 \mathrm{~mm}$. Specification for this lens can be seen in Appendix B. Full collimation however would not be possible because the focal point of the $\mathrm{X}$ and $\mathrm{Y}$ axis of the beam do not focus at the same point, as discussed earlier. This means that the beam exiting the side scatter lens would create an ellipse. It would be possible to fully collimate the light but this would require two lenses with different focal lengths in the $\mathrm{X}$ and $\mathrm{Y}$ direction.

A focal mirror was also inserted into the flow cell to reflect light back that came off in the opposite direction of the side scatter. The goal of this mirror was to increase the side scatter intensity light. This mirror was glued into the cavity on the side of the flow cell. All the side scatter light is passed through the optics truss, which was created using SLM 3D printing. This truss contains dichroic mirrors, filters, and focusing lenses. The resulting truss skeleton looked like that seen in Figure 22. 


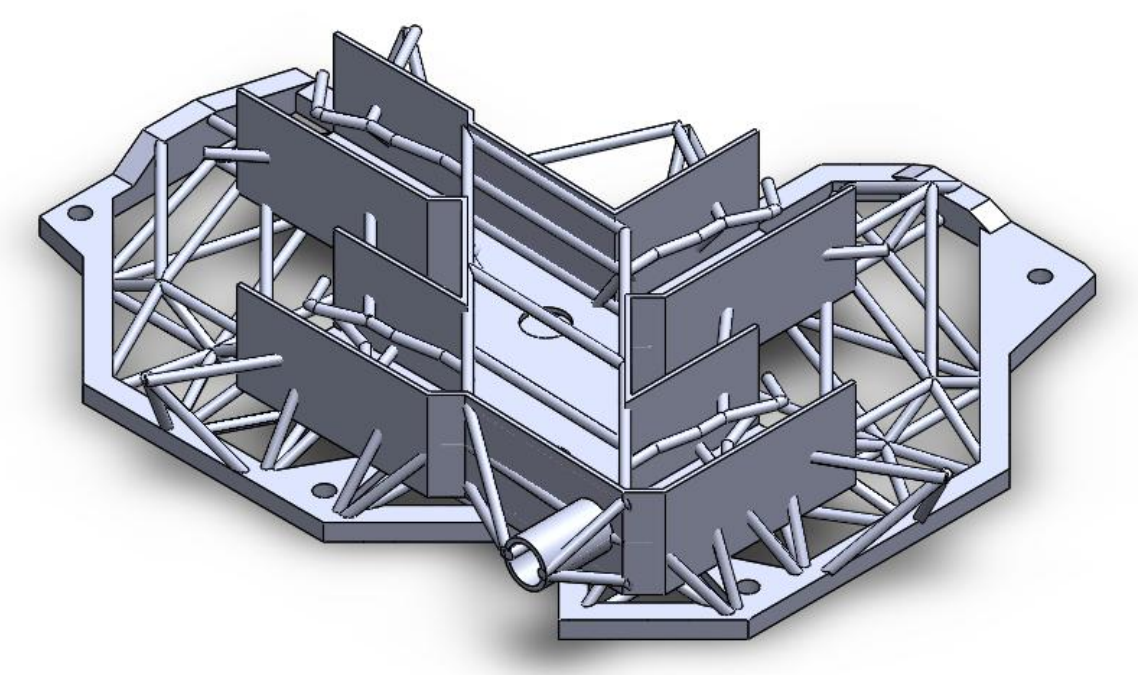

Figure 22. Optics truss skeleton without holders

The truss was created to hold all of the components for the side scatter and fluorescence detection system. Work went into this truss to ensure that all the geometries were correct for the light traveling through the truss. All the mirrors were placed at 45 degree angles to one another to ensure that the light approach the mirror perpendicular to its exit.

Channels were also shifted to account for the thickness of the mirror being placed in front of the channel. This shift was calculated using the second law of refraction. This states that light passing through a medium at an angle will shift laterally from entrance to exit of that medium. The distance between the focusing lenses and pinholes was also carefully looked into, which will be talked about in more detail later. This was to ensure that the small adjustments of the lenses could create a light path that would pass through the small pinhole fixtures. Once the skeleton of the truss was created it was populated with the dichroic mirror, filter, and focusing lens holders. 
The dichroic mirror holders are responsible for holding the dichroic mirrors in place. The design of the holder and location that they are inserted into the truss are seen in Figure 23.
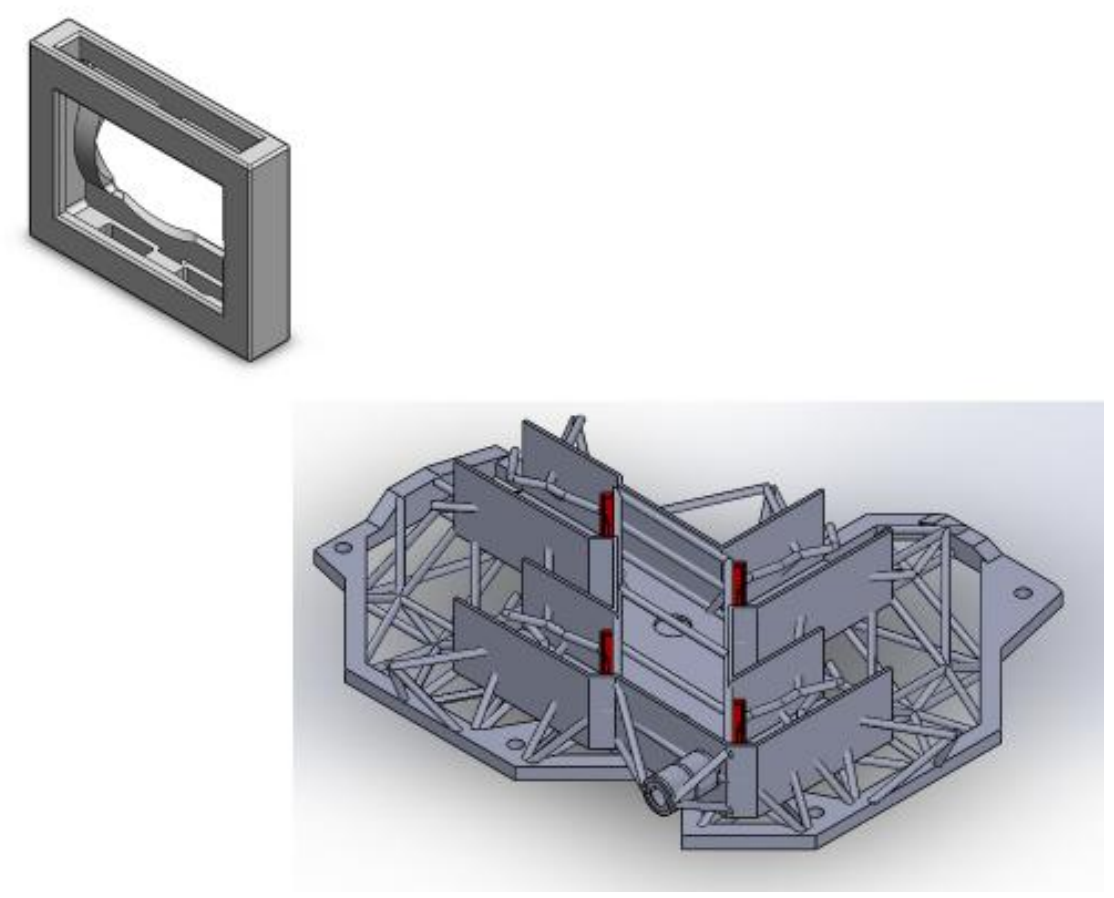

Figure 23. Dichroic mirror holders

The mirrors were then pushed into their holders. The holder is built in such a way that it will flex to allow the mirrors to be inserted, then push the mirror flush to the face of the holder. This is to ensure that the mirrors are placed into the holder correctly and make sure they are aligned at a 45 degree angle. The type of mirror that was added into the truss was different depending on the FL channel that it was in front of. This was to collect different wavelengths for each channel. 
Filter holders were then placed behind these mirrors. These would be used to hold filters that would help to eliminate more unwanted wavelength light in the channel. These can be seen below in Figure 24 .
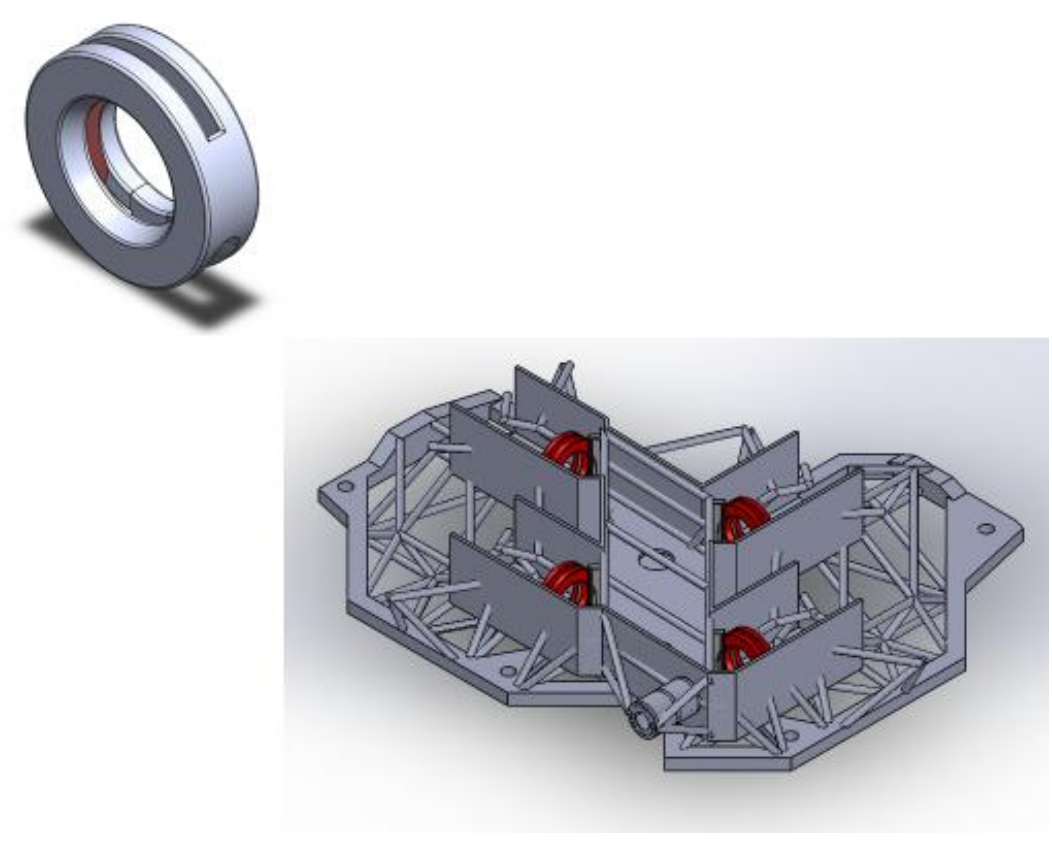

Figure 24. Filter holders

The filters were also pressed into the holder from the top much like the mirror holders. These holders allowed filters to be placed into the truss system. Once the filters were pushed into place they pressed firmly against the back of the filter to hold it flush against the front surface.

Focusing lens holders were then placed into the truss. These are threaded and allowed the focusing lens to be moved in and out of the holder changing the focal length of the lens depending on its position in the holder seen below in Figure 25. 

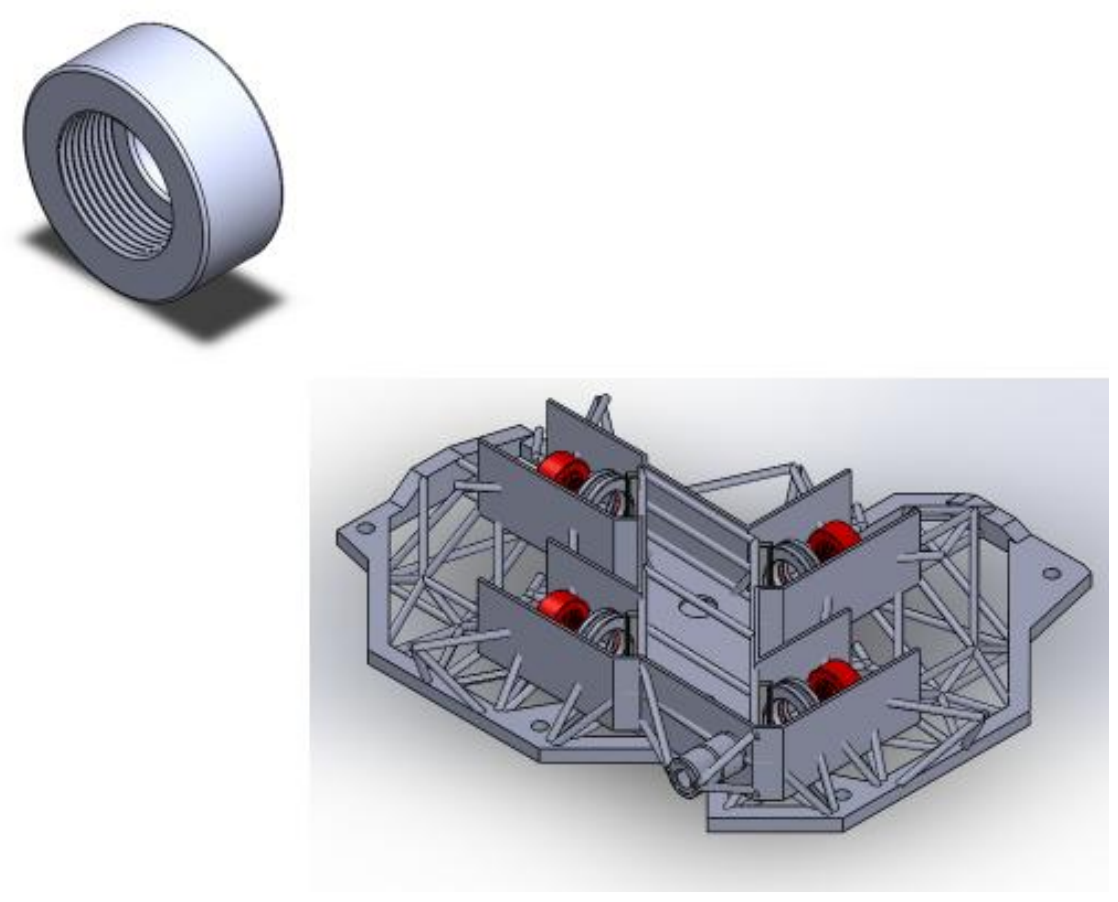

Figure 25. Focusing lens holders

The lenses for this were threaded into the truss. The focal length of the lenses was used in determining how far the lens should be from the pinhole.

Pinholes were then added to the truss. These pinholes were used to reduce the amount of environmental light that could be passed into the detector. The pinholes were also used as a way to set the focusing lenses for the FL channels. The laser light should be able to pass through the pinhole without being hit by the light. They can be seen below in Figure 26. 

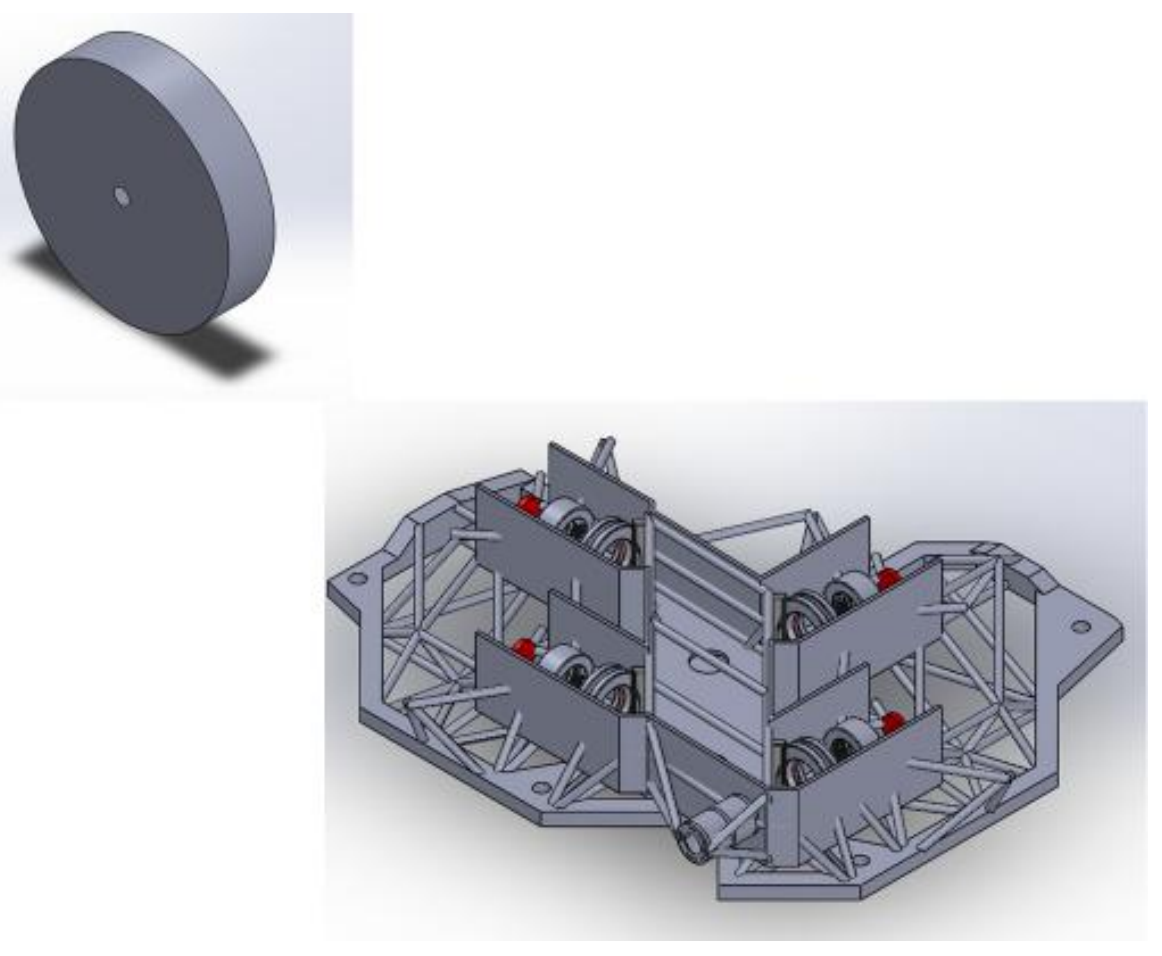

Figure 26. Pinholes

The pinholes were designed to give a clean light shape entering into the PMT. The front of them gave a sharp circle while the back had a cone taken out of it leading up to the circle. This allowed for light that passed through the circle to not hit the pinhole because of its thickness. This also allowed for the removal of less material if the pinholes needed to be given a larger diameter.

Finally, PMT mounts were placed into the assembly. These mounts created a surface that the PMT's could sit on and be bolted to. The PMT holders can be seen in Figure 27. 

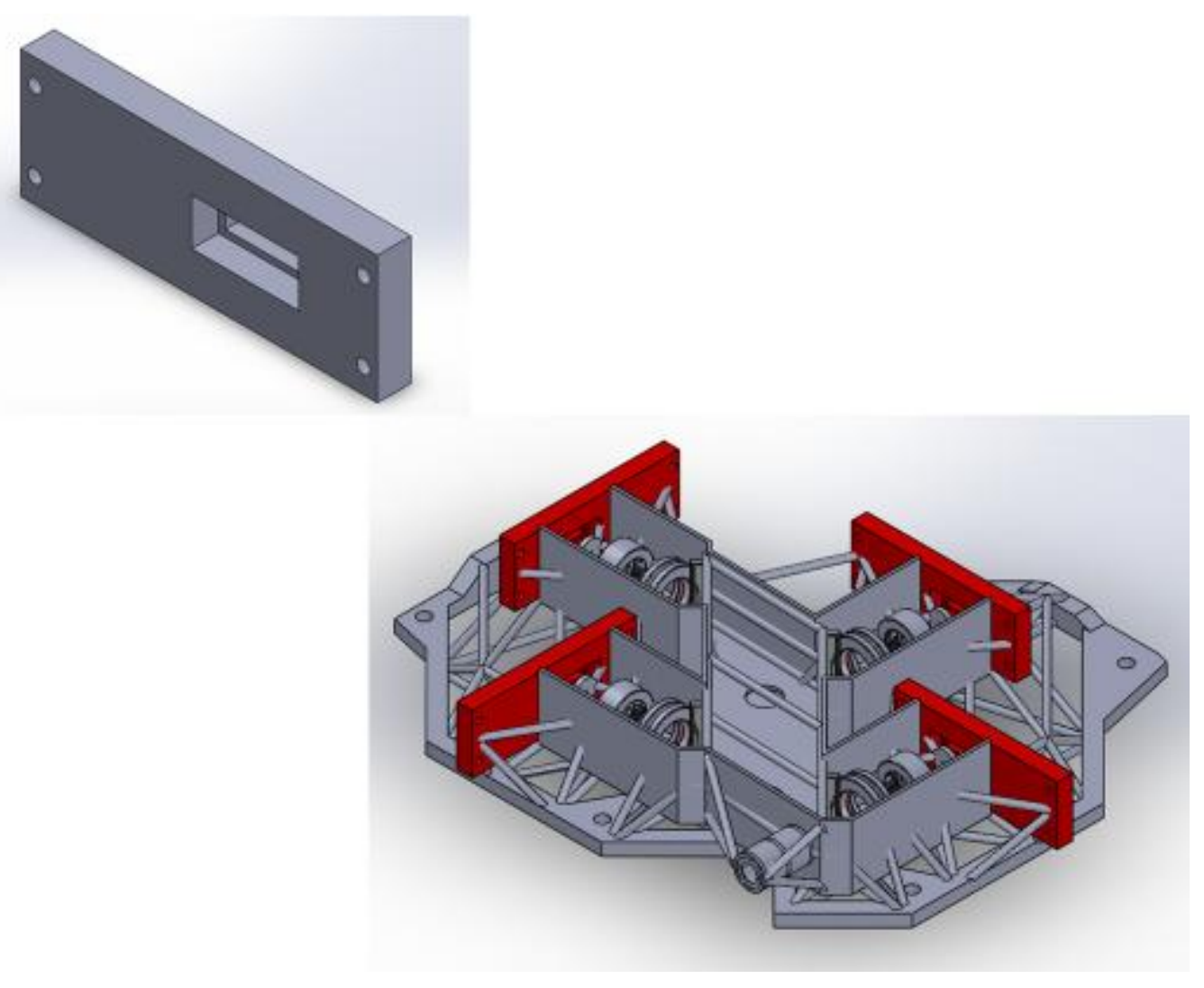

Figure 27. PMT mounts

The PMT mounts had holes that allowed for screws to be threaded into the PMT that sat behind it. These also had a feature that protruded from the back side. The allowed for the mount to sit somewhat inside the window of the PMT. The aim of this was to reduce environmental light entering into the PMT.

\section{Dichroic Mirror}

The dichroic mirrors are then placed into the mirror holders. These are responsible for directing the light down the correct channel. All the dichroics are $15 \times 17 \mathrm{~mm}$ with a thickness ranging from $1 \mathrm{~mm}$ to $2 \mathrm{~mm}$. These dichroic mirrors were purchased from Chroma Technology Corporation. The table below, Table 2, shows the breakdown of the short-pass dichroic mirrors that were placed in each channel. 
Table 2. Dichroic mirror type and position

\begin{tabular}{|c|c|}
\hline \multicolumn{2}{|c|}{ Dichroic Mirror } \\
\hline Fluorescence Channel & Mirror Type \\
\hline Side Scatter & $500 \mathrm{~nm}$ short-pass \\
\hline FL1 & $565 \mathrm{~nm}$ short-pass \\
\hline FL2 & $615 \mathrm{~nm}$ short-pass \\
\hline FL3 & $745 \mathrm{~nm}$ short-pass \\
\hline
\end{tabular}

If different wavelengths of light were needed in each channel these could also be replaced to capture wavelengths that were needed. For spec sheets on the dichroic mirrors please refer to Appendix C.

\section{Filter}

The filters were then loaded into the filter holders. These are responsible for cutting down excess light that passed through the dichroic mirrors. All of the filters have a diameter of $12.5 \mathrm{~mm}$ with a thickness of $1 \mathrm{~mm}$. The filters were purchased from Chroma Technology Corporation. Table 3 below shows which filters were placed into which corresponding channel. 
Table 3. Filter types and position

\begin{tabular}{|c|c|}
\hline \multicolumn{2}{|c|}{ Filter } \\
\hline Fluorescence Channel & Filter Type \\
\hline Side Scatter & $488 \mathrm{~nm} \pm 5 \mathrm{~nm}$ \\
\hline FL1 & $525 \mathrm{~nm} \pm 25 \mathrm{~nm}$ \\
\hline FL2 & $595 \mathrm{~nm} \pm 25 \mathrm{~nm}$ \\
\hline FL3 & $685 \mathrm{~nm} \pm 35 \mathrm{~nm}$ \\
\hline
\end{tabular}

The filters could also be changed to match certain wavelengths needed in that channel.

Please see $\mathrm{D}$ for specifications on the filters.

\section{Focusing Lens}

Finally, the focusing lenses that were used to focus the light on the PMT detector were inserted into the truss. The lens was purchased through ThorLabs and is seen below in Figure 28 .

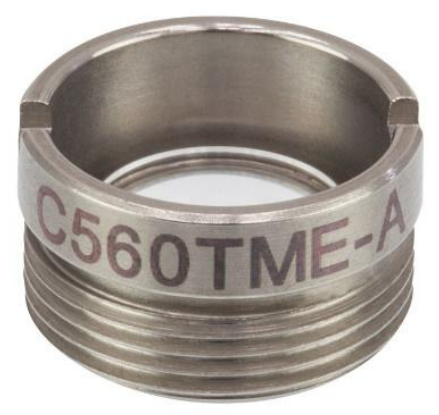

Figure 28. Optics truss focusing lens 
The threaded design of the lens and the holder allowed for the lens to be adjusted in very minute amounts. Using the feature allowed for adjustments of the focal depth that the PMT would see. For further specs of the focusing lens please see E.

\section{Forward Scatter Diode}

The detector that was used to detect forward scatter was a diode. The forward scatter diode is an electronic board that has a diode located on it. The light would hit the diode and be processed by the electronic board it is connected to it. The board takes in the light from forward scatter, amplifies it, and processes it to be passed onto the software package. This board was created by Terry Boyd a contract worker for CytoFlow.

PMT

The particular PMT used in this project is the H9305-02 version made by Hamamatsu. This PMT offers a gain of $10 \mathrm{e}(7)$ and has a working wavelength range of $185 \mathrm{~nm}$ to 750nm. This offers an optimal range for all wavelengths that the CytoFlow device is expected to see. Alternate PMT's were used in this project for early testing due to availability and time constraints. Further specifications for H9305-02 PMT can be seen in $\mathrm{F}$.

\section{Preamp}

For further amplification of the signal seen by the PMT a preamp was used. These preamp boards were created by Terry Boyd of in2being. The preamp boards contained power for the PMT as well as amplification of the PMT signal. The boards were 
designed to be able to adjust the amplification in two different ways. A resistor could be changed on the board which could allow for more or less sensitivity. A potentiometer was also place on the boards. This was a screw that allowed the amplification to be changed with a much smaller range than changing the resistor. 
Setting up the Machine (Alignment)

Alignment of the laser light pathway is very crucial to the results that the cytometer will produce. Various different steps were used to ensure that the cytometer was aligned properly. Installation was done in much the same order as the light travels through the machine. The flow cell was first placed into position on the ground plate of the cytometer.

\section{Forward Scatter}

First the forward scatter assembly was created and put into place this included the laser, the flow cell, and the forward scatter block. As mentioned previously the laser had to be placed $30 \mathrm{~mm}$ from the capillary. Once this was done the perpendicular alignment of the laser to the capillary had to be adjusted. By looking at the projection caused by the laser passing through the capillary and projecting both onto the forward scatter lens and the projection on a wall the laser was aligned. This was done by looking at both the forward scatter lens and wall projection. Once the projection was symmetrical on both the $\mathrm{X}$ and Y axis, as seen in Figure 29, the laser was bolted into place. 


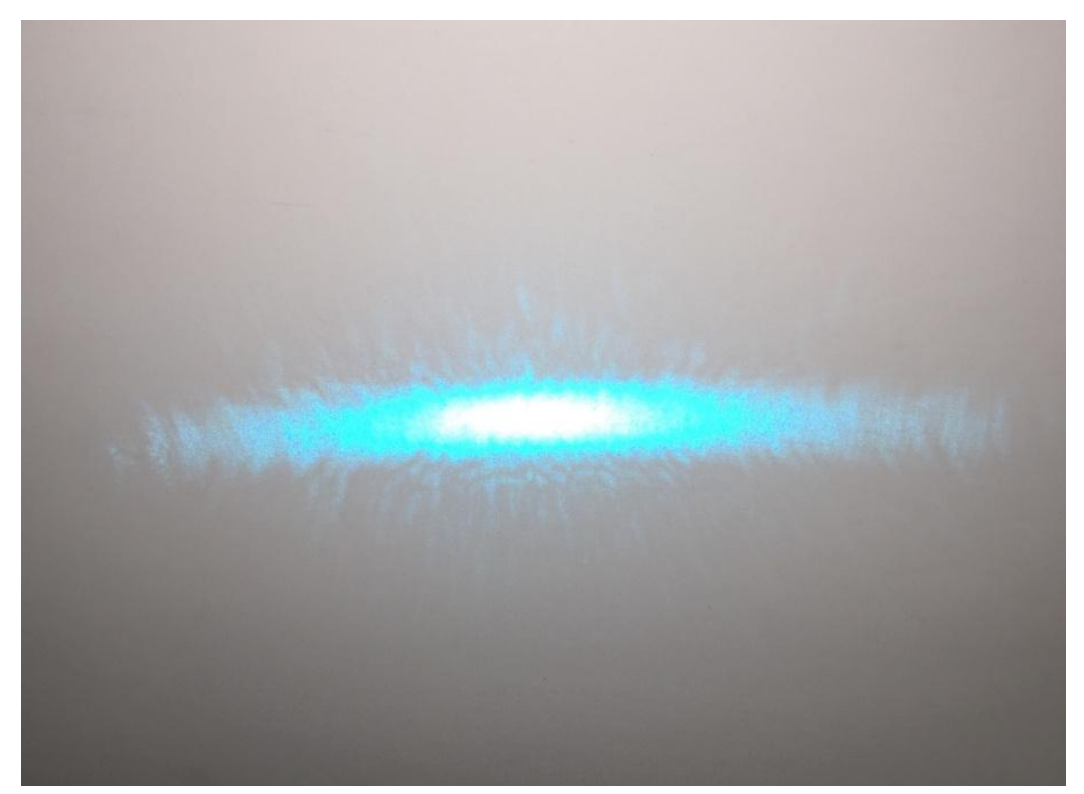

Figure 29. Laser going through capillary

As seen the projection of the laser created a profile. This profile is symmetrical across both the $\mathrm{X}$ and $\mathrm{Y}$ axes. Once this profile was seen it was known that the laser was hitting the center of the capillary.

A piece of electrical tape was used to create the beam blocker for this iteration. Using the projection created on the wall the tape was positioned and cut until the laser light was mostly covered and only an aura of bouncing light was projected onto the wall. The result of the beam blocker can be seen in Figure 30. 


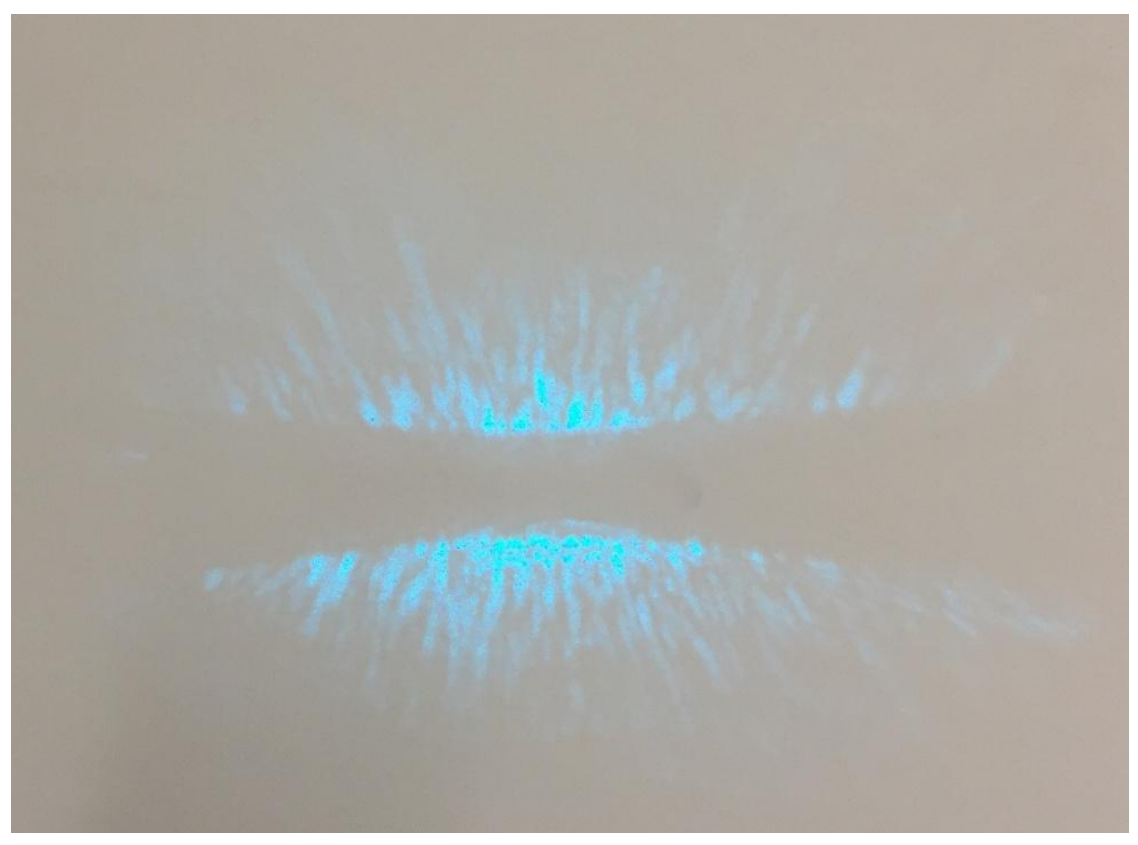

Figure 30. Laser going through capillary and hitting beam blocker

The new projection was to look like that seen in the image above. By covering most of the projection of the laser it would be easier for the detector to determine the deflection of light off the cells. Once forward scatter alignment was correct the truss was populated with dichroics, filters, and focusing lenses so that side scatter alignment could commence.

\section{Side Scatter}

In order to align side scatter the forward scatter alignment first had to be optimal. Once this was completed the truss was aligned off the side of the flow cell. Fluorescein dye emitting in the yellow wavelength when excited by a 488 light wavelength was used to help with this position. The fluorescein light that came off the side scatter was so intense that it could be seen propagating through the truss. The truss was then manipulated in 
such a way that when the light passed through the dichroics, filters, and out the pinholes which created a circle when passing through correctly. Once this was completed the truss was bolted into place and the PMT's were placed onto the truss.

\section{Data Optimization}

Once alignment of the system was optimal the system could be evaluated by looking at the data it produced. This was done by looking at key statistics the software produced based off data recordings. To create these data recordings beads purchased from Spherotech were used. Specs can be seen in Appendix G. These beads have known size and color. As all data is known about the beads the software should produce the expected values when bead data is run through the machine. When looking at these beads FSC is expected to create one slim peak. This is because all the beads are the same size. The fluorescent channels shall report back 8 peaks. This is because the beads are made up of 8 different populations of beads each with its own percentage of fluorescent marker. The image below Figure 31 shows some of the statistics used to determine if a machine is performing correctly. 


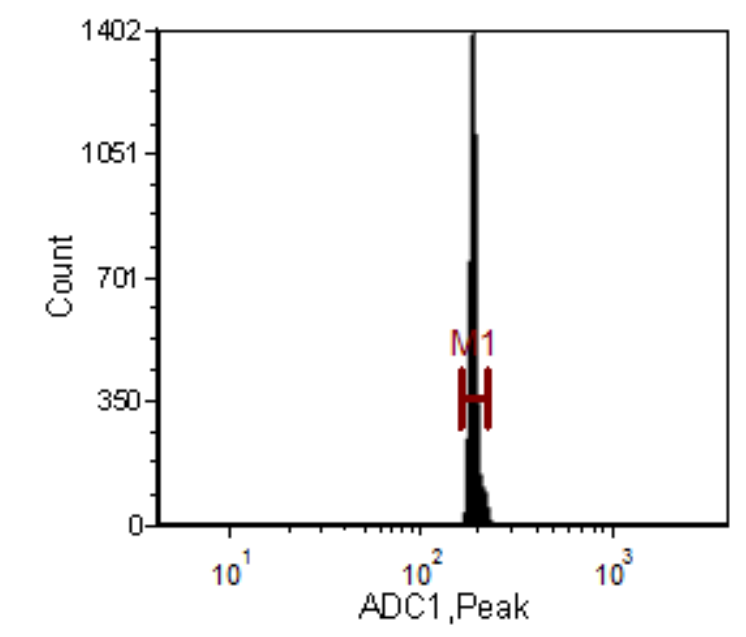

\begin{tabular}{lll|ll|l|l|l|}
\hline $\begin{array}{l}\text { His togram } \\
\text { \# }\end{array}$ & Filename & Parameter & $\begin{array}{l}\text { \# of } \\
\text { Events }\end{array}$ & $\begin{array}{l}\% \text { of } \\
\text { gated } \\
\text { cells }\end{array}$ & Median & CV \\
1 & April 1 BLR Enabledr.fCs & ADC1,Feak & 19716 & 98.58 & 181.00 & 5.04 \\
\hline
\end{tabular}

Figure 31. Forward scatter software data

The image is taken from a software analysis tool. This tool was used to look at the forward scatter channel of a cytometer and produce statistics based off the data points seen.

The key statistics used over the period of testing were used to look at the intensity of the signal, which refers to the location on the $\mathrm{X}$ axis, and the control variance (CV) of the peaks. If the intensity of the signal is high it will produce data higher up on the $\mathrm{X}$ axis, while if it is low it will produce data lower on the $\mathrm{X}$ axis. The $\mathrm{CV}$ is a percentage that is essentially the repeatability of a data point. If the machine is optimized it will have a low $\mathrm{CV}$ percentage. Meaning the peak the bead produces will land at the same intensity every time. If a $\mathrm{CV}$ is high it means that the bead has differing intensities which implies that the repeatability of the reading is low. Below is an image of a good CV and a bad $\mathrm{CV}$ in Figure 32. 

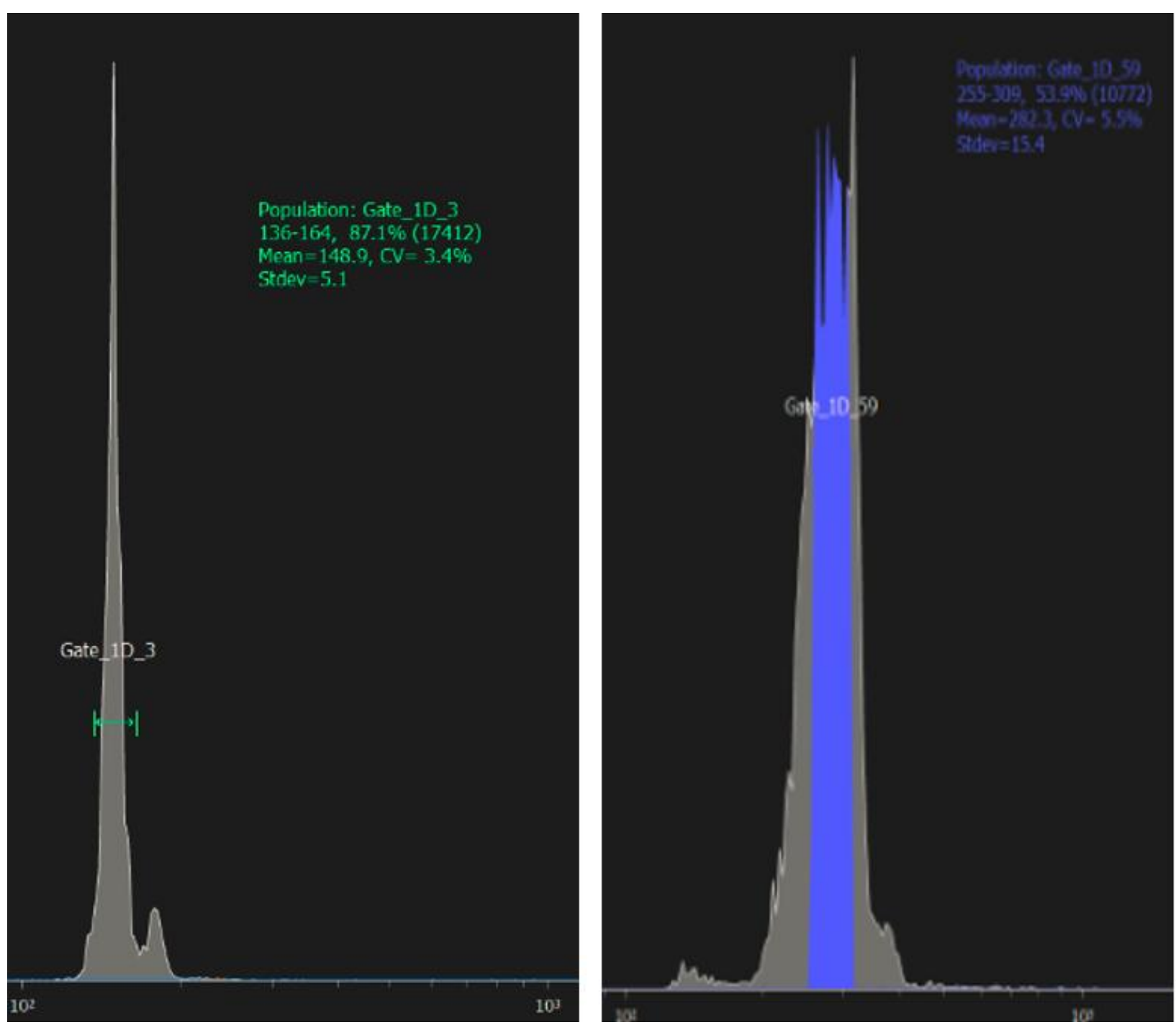

Figure 32. Screenshots of forward scatter data CV of 3.4\% (left) CV of 5.5\% (right)

Both of these images were taken from the same machine and looked at the forward scatter channel. The image on the left represents a peak with good CV of 3.4\%. While, the image on the right represents a bad CV of 5.5\%. This means that the machine that created the image on the left produced better results by having a greater reproducibility of 
each cell signal. The change in the two readings was created by adjusting the position of the optics. 


\section{Iteration 1 Data}

In order to produce known results 8 peak beads purchased from Spherotech were used to collect data on the machine. These beads act as markers to produce 8 different intensities of light. They range from a bead that has no marker in it to a bead that has a high percentage of marker in it. There are a total of 8 different bead populations in a vial containing these beads. Each bead correlated to a different intensity when hit with light. These 8 different particles produce different voltages in the PMTs thus creating 8 different peaks in the software.

This iteration of the machine served mainly as a proof of concept that data could be collected in this manner. Once data was able to be seen to ensure that data was coming through all the channels optimization and peak resolution became the aim of the project.

\section{Iteration 1 Flaws}

The first areas to be optimized after viewing the data produced by the initial iteration were: laser choices, correcting dichroics/filters, and optimizing the side scatter lens. As seen by some issues with the data it appeared that there was a problem with how the beads were passing through the laser light. Light pathway issues were likely caused by off the shelf dichroics and filters. It was possible that creating custom optimized dichroics and mirror could help to reduce the amount of noise in the bead signals and help to create better data signal. Since the side scatter light could not be collimated again with the current setup it was not clear where the focal length of the lens to capillary should be placed. 


\section{Machine Comparison}

To continue on the proof of concept experimentation, a test was conducted to compare a working system to CytoFlow's system. For reference the old system is known as "Yahoo" and the system CytoFlow is using is known as "Azurite". This experiment was completed using the software of the Yahoo system as well as the PMT that worked with it.

\section{Objective of machine comparison}

The goal of this experiment was to examine how the FL channels were affected by the different set up constraints: the collection PMT and supporting electronics, and the position of the PMT. The Yahoo system was used in this experiment as a benchmark to compare against the Azurite system. Various positions of the PMT were compared to determine if signal degradation was related to the collection optics.

\section{Results of machine comparison}

First, baseline readings were taken directly off the flow cell collection optics. This position represents the best possible fluorescent signal from the current flow cell and laser. This was accomplished by the setup seen in Figure 33 below. This experiment was done using the FL2 filter placed in front of the PMT. 


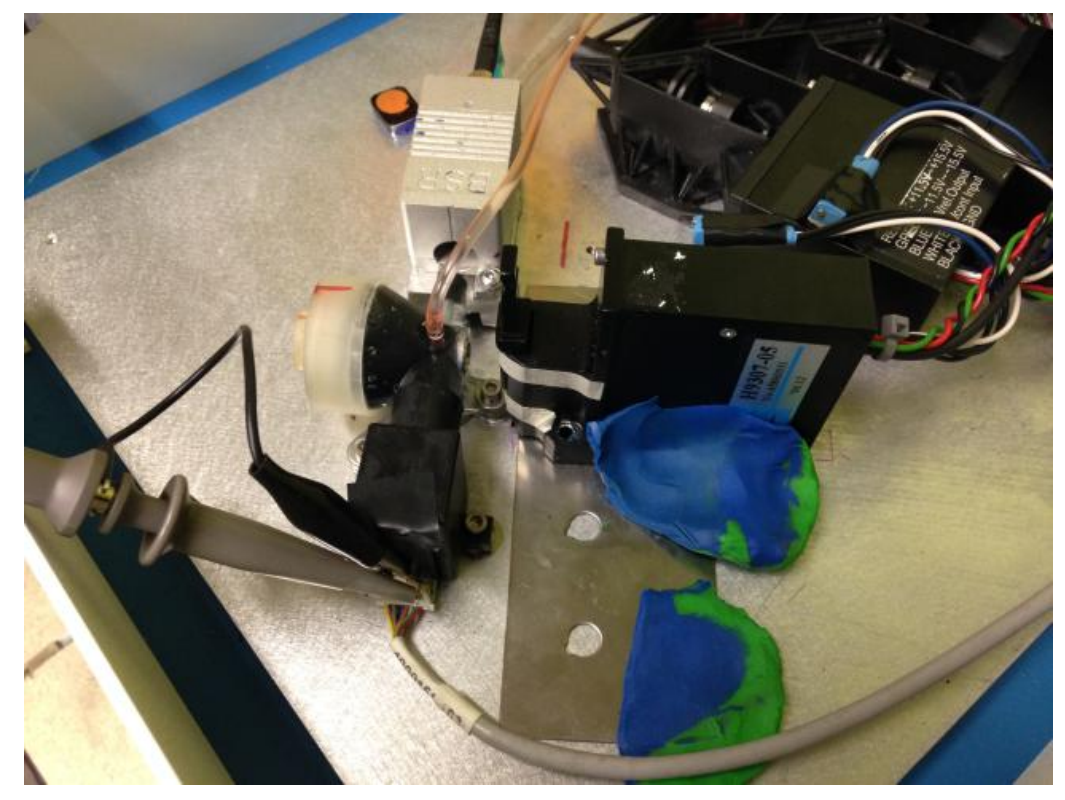

Figure 33. Baseline flow cell data collection

The first experiment was to look at the difference in intensity produced by adding a side scatter mirror. Data was collected with and without the mirror installed. This was done to see if the mirror was helping or hurting data collection. The results can be seen below in Figure 34 and Figure 35. To create this data the 8 peak beads were used. If the optics were producing optimal results 8 peaks would be seen in the software.
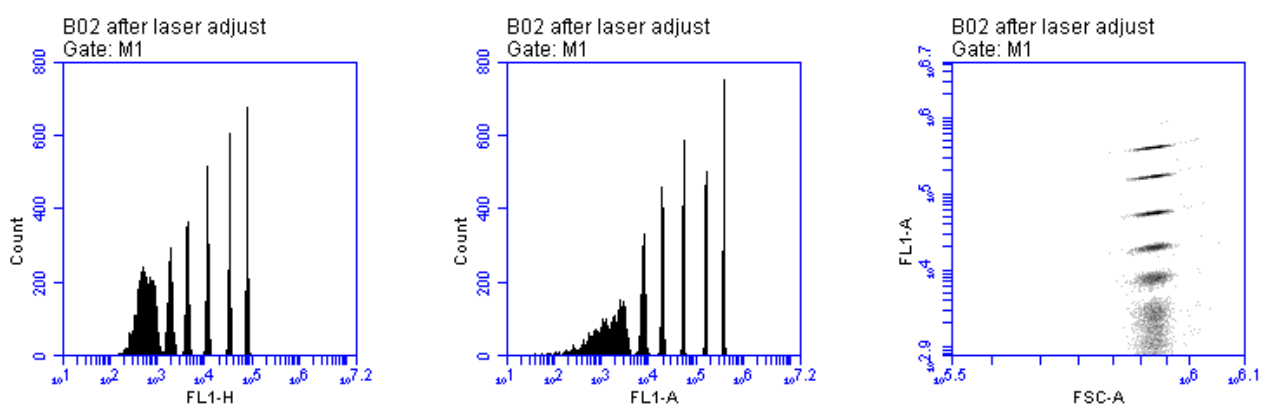

Figure 34. Baseline readings without the mirror 
Without the mirror the top peaks intensity can be seen. This data is looked at using the PMT situated just off the flow cell.
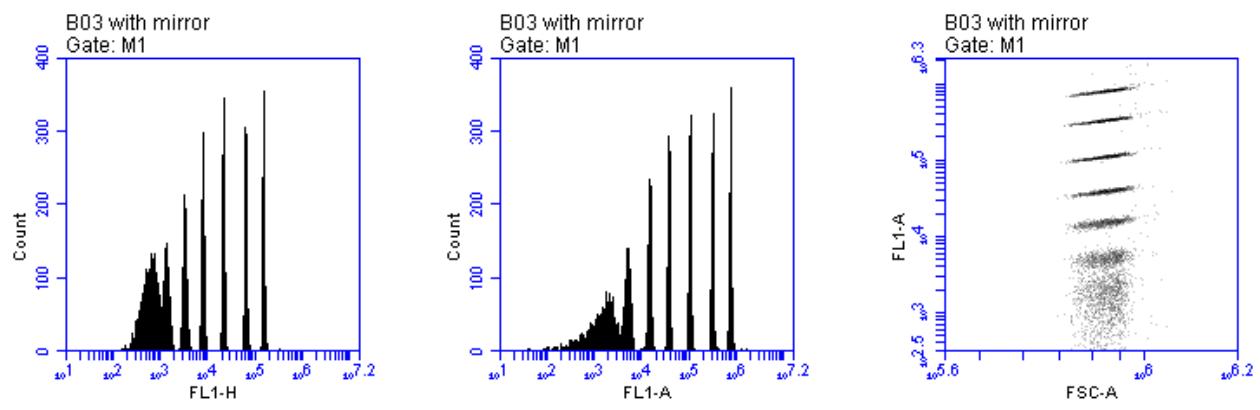

Figure 35. Reading with mirror in place

As seen compared to the baseline the intensity of the signal increased when the mirror was put into place. This showed that the mirror was helping the system by increasing the intensity.

Once the data had been collected the complete cytoflow setup was put back in place. The truss was manipulated until the top PMT performance was achieved. A series of experiments were completed to analyze the optics of the truss and how data collection was affected by different variables. The first test was completed by placing the Yahoo PMT in the FL2 location with FL2 filters in place. The results can be found in Figure 36. 

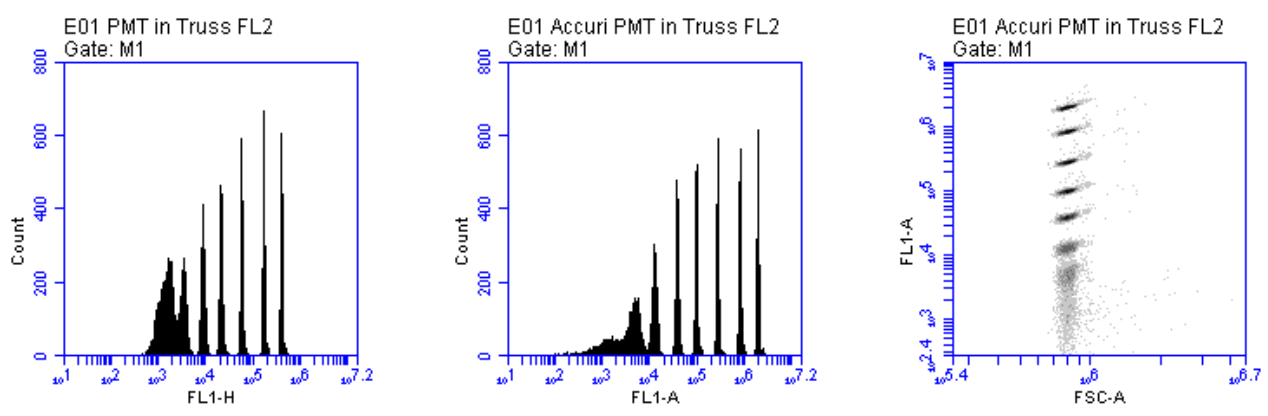

Figure 36. FL2 Location FL2 Filters

The above images show FL2 data taken off the Yahoo PMT and software. This gives about 7 peaks of the 8 that should seen.

The same method was then used to collect data from the FL1 location with the FL1 filters. The resulting data can be seen in Figure 37.
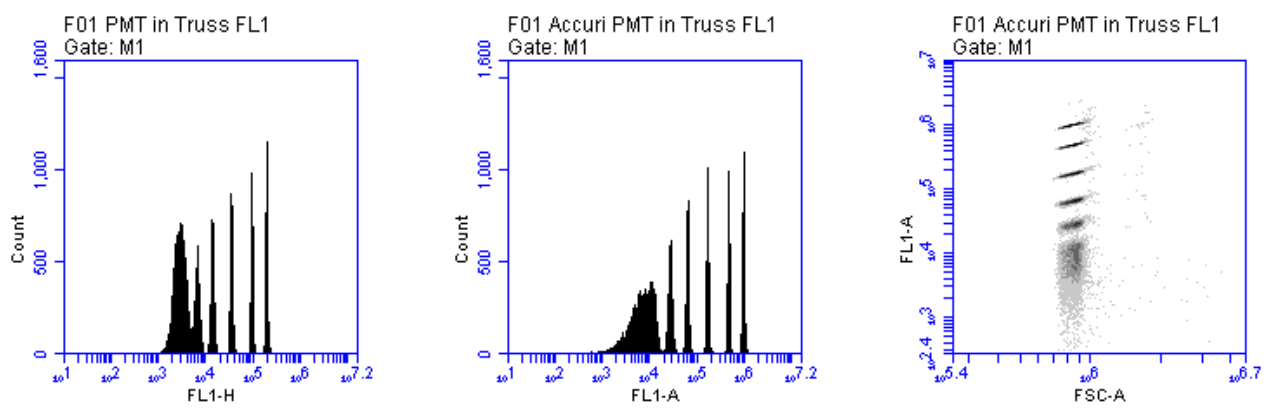

Figure 37. FL1 Location FL1 Filters

In FL1 only 6 of the 8 expected peaks could be seen on the Yahoo PMT and software.

Then different setups were analyzed and data was collected, the readings were taken by moving the PMT, the filters, and the dichroic that accompany the correct FL readings. 
Since the truss was moved in this process, new baseline readings were taken followed by the experimental data collection. The baseline recordings can be seen in Figure 38 .
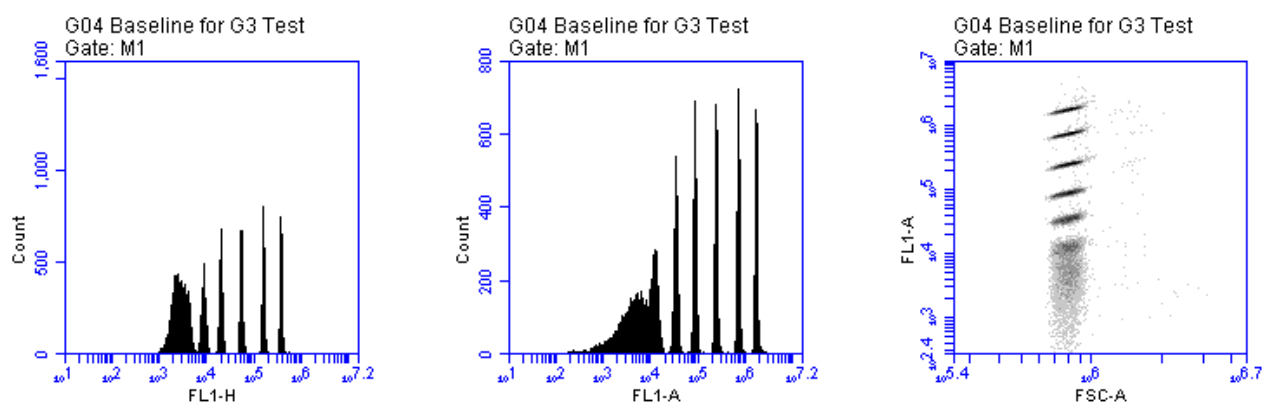

Figure 38. Baseline FL2

Baseline recordings had to be taken off the Yahoo system. The setup yielded 6 peaks on the Yahoo software.

\section{$\underline{\text { Conclusion }}$}

The addition of the side mirror in the flow cell helped to increase the signal that was seen in fluorescent channel 2. This conclusion is illustrated in the comparison between Figure 34 and Figure 35. It was also found that the current optics truss is capable of producing results that are comparable to the results seen when FL data is collected right off the side of the flow cell. This means that with the experiments conducted the optics truss did not degrade the flow cell side signal. In this experiment it was not known if the H9305-02 and H9307-05 PMT's on each software setup were comparable. It could not be said at the time if the PMT or software was causing the change in readings when looking at Yahoo or Azurite. Mark Naivar, of DarklingX, had stated previously that the PMT being 
used with the Yahoo system is more sensitive to the wavelengths being viewed. This would account for poorer resolution of 8 peaks in the system. 


\section{PMT Testing}

Once a new PMT of the same version that was used in the Yahoo experiment was installed, data was collected that compared the two PMTs. The below image is of the H9307-05 PMT that was in use in the Azurite system. The FL2 channel is seen in Figure 39 below. This data was also collected using 8 peak beads.

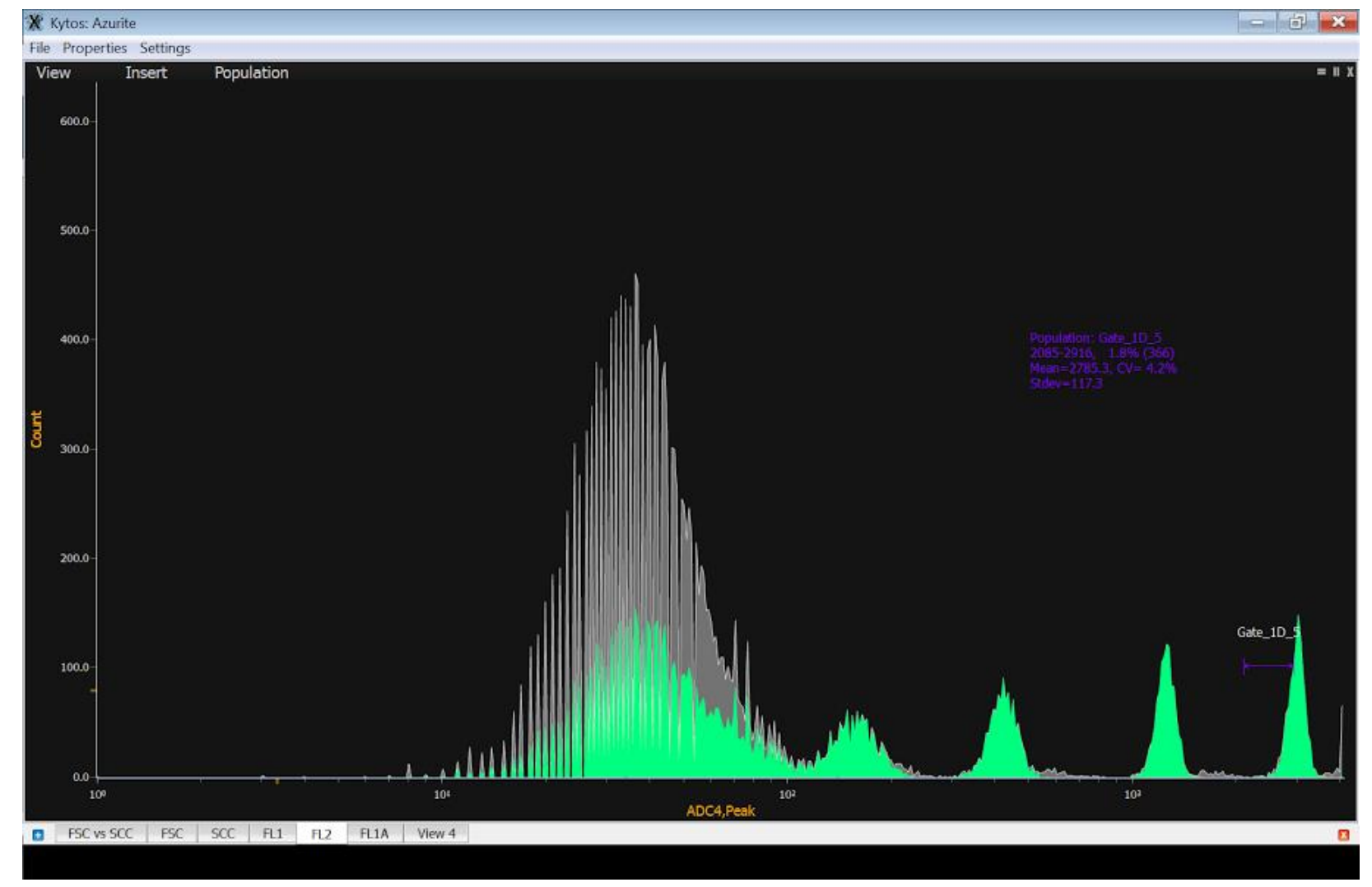

Figure 39. 8 peak data taken from FL2 on H9307-05 PMT

Using the H9307-05 five distinct peaks can be seen. Three more peaks still had to be resolved to produce the expected bead populations.

The new PMT that was previously used in the Yahoo setup was then placed on FL2 and the data was recorded. This is PMT H9305-02, the data can be seen below in Figure 40. 


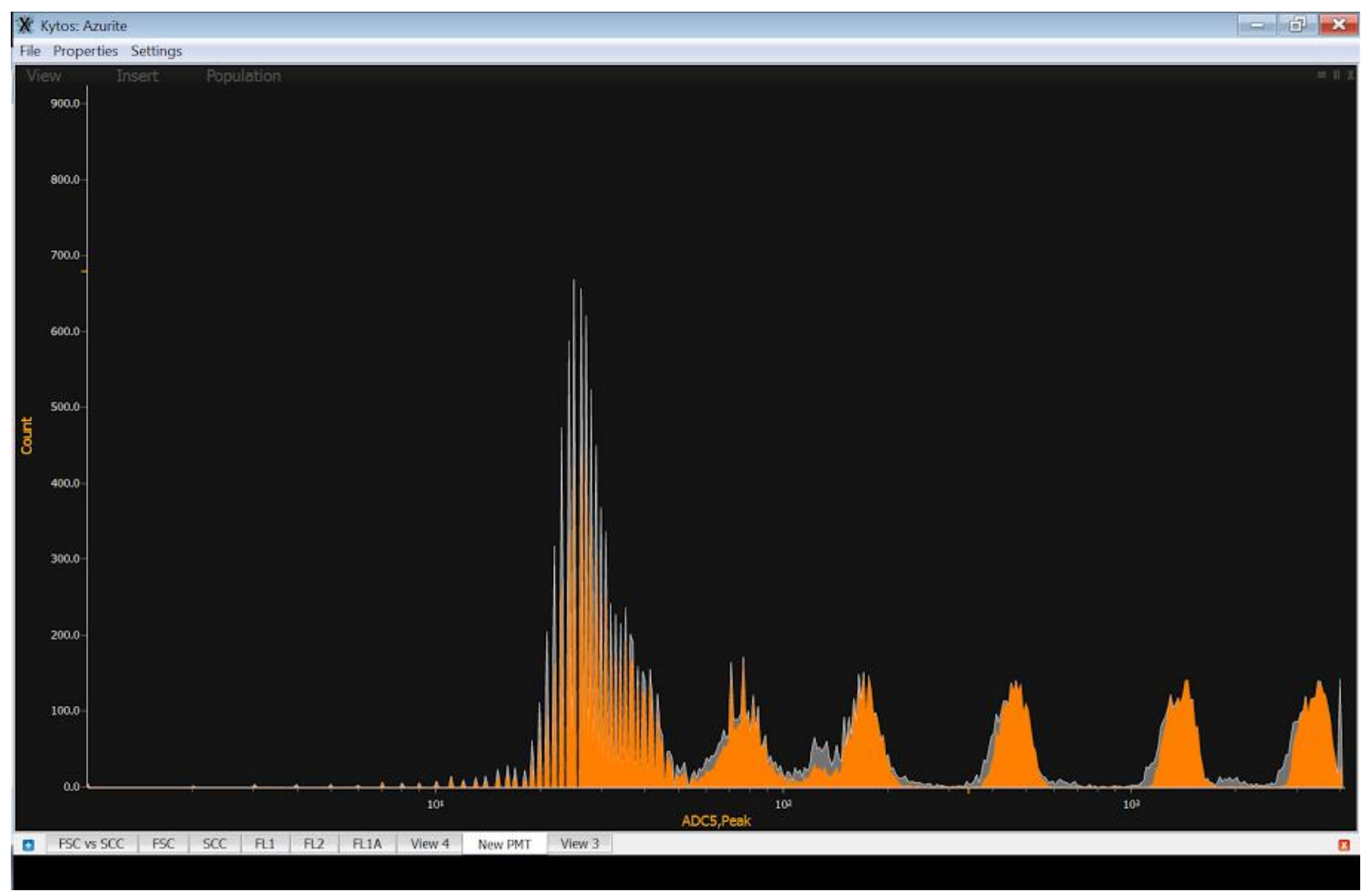

Figure 40. 8 peak data taken from FL2 on H9305-02 PMT

In this image it is clear that 6 distinct peaks can be seen. This is still not the full 8 peak resolution that is needed but it produced one additional peak than the PMT before it.

Simply by comparing the two data images it can be seen that the H9305-02 PMT was able to resolve another peak in the data. This shows that the Azurite system is not optimized based on the correct PMT to use. Two of these PMT's were acquired and installed into the Azurite system. These 2 PMT's were placed at FL1 and FL2. As SCC did not need as much resolution the H9307-05 PMT was placed there as none of the other PMT's were available and they had a long lead time associated with them. 


\section{Data Resolution Testing}

Further experimentation was done using the Yahoo software. As this system is a 24-bit system it has more room for data to be stored on the $\mathrm{X}$ axis. The Azurite system is only 12-bits which causes less resolution. If 8 peaks were able to be accomplished using the Yahoo system that would then verify that all 8 peaks could be obtained using our optics setup. The data was collected off of FL2 because at this point it gave us the best resolution. Only $1 \mathrm{FL}$ channel was able to be recorded because only 1 PMT was able to be integrated with the Yahoo system. An 8 peak bead was run and the resulting data can be seen in Figure 41 below.

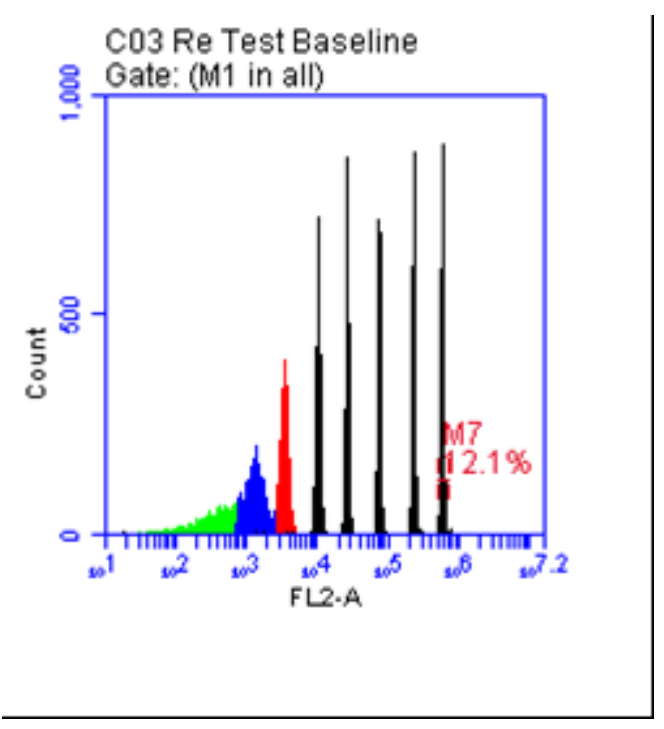

Figure 41. 8 peak data taken from FL2 on Yahoo system

The data seen above is FL2 taken off the Yahoo system. This concluded that the truss and all optics were working correctly as all 8 peaks could be seen in this file. This also showed that the reason all 8 peaks couldn't be seen thus far was related to software. Much work would then have to go into making the data look like this on the Azurite 
software. This would be done by cleaning up all noise in the system and optimizing all components.

\section{Iteration 2 Experiments}

This iteration of the optics assembly looked to combat some of the problems seen with the first build. Experiments included laser shape testing, changing of optical filters, and side scatter lens testing.

\section{Laser Shape Testing of iteration 2}

It was a thought that the waist shape of the laser could be at question with the current system. Results of the testing were based off the CV's, peak resolution, and intensities of the light seen by the detectors. The experiment was conducted by first recording a baseline then using different size convex planar lenses the signals of each detector was analyzed. The baseline data for this experiment is seen below. The data is looked at by FSC, FL1, and FL2 respectively these images can be seen in Appendix H. This data created a starter for the other testing to be compared to. The signals in the baseline gave a good reading for forward scatter, 4 peaks in FL1, and 5 peaks in FL2.

Once the baseline control sample was taken, the shape of the beam could then be changed for testing. This was done by placing the plano convex lens in front of the laser. Doing this caused the beam waist in the $\mathrm{X}$ direction to be wider. The first lens tested had a focal length of $100 \mathrm{~mm}$. The results are seen in the FSC, FL1, and FL2 respectively these images can be seen in Appendix H. These results created a decent looking forward 
scatter if the excess noise was ignored. It did however reduce FL1 to 2 peak and FL2 to 3 peaks.

The same experiment was then done using a lens with a $200 \mathrm{~mm}$ focal length. The results are seen with FSC, FL1, and FL2 respectively these images can be seen in Appendix H. This setup also created decent forward scatter if the noise was ignored. However, it also decreased the peaks seen in the fluorescent channels to 1 peak in FL1 and 3 peaks in FL2.

The CV's and intensities did not need to be analyzed for this experiment because less peaks were seen in this data, meaning no matter what the data was not as good. A quick look at the reduction in peaks seen in each channel revealed that changing the beam shape did not help the system. However the validity of this experiment was called into question due to human error. This experiment was completed by roughly lining up the lens with the laser. If the lens was not lined up perfectly to where the laser passed through the center line of the lens that would cause the beam to shift left or right which could cause the beam to miss the center of the capillary. Further exploration would go into this topic to be discussed in redesign 2.

\section{Optics Filtering of iteration 2}

New customized filters and dichroics were ordered for the new iteration of the device. The results were examined by looking at the CV's and peak separation in the data. The baseline of the machine with the original filters and dichroics can be seen below in Figure 42. 

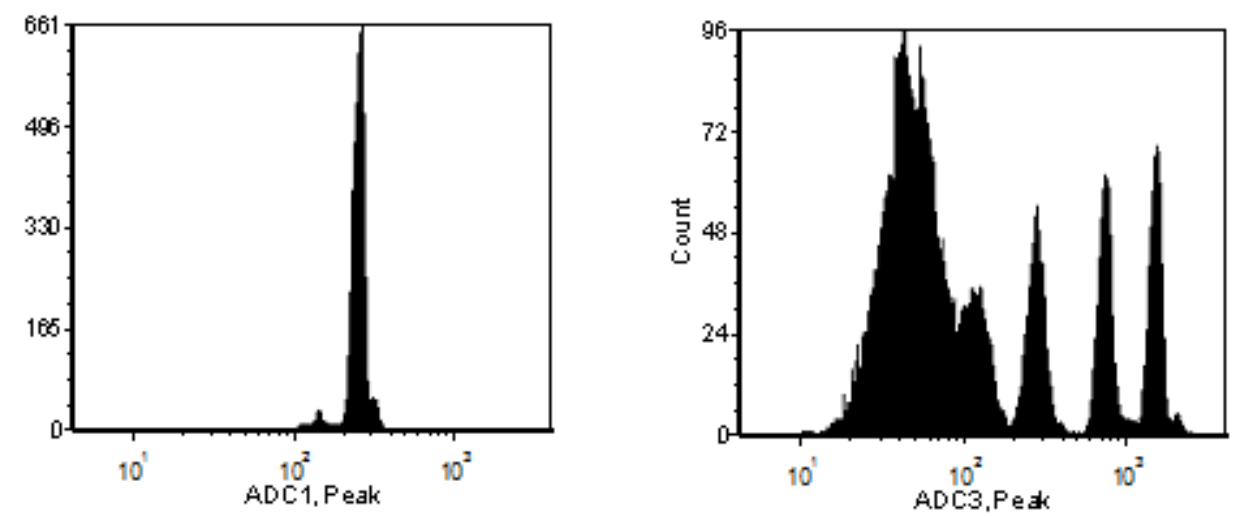

Figure 42. Baseline of optics FSC (left) FL1 (right)

This data shows a good looking forward scatter on the left with about 5 peaks seen in the FL1 channel. At this point more peaks still needed to be resolved and the peaks needed to have a better separation so they did not interfere with one another. The new optical pieces were then placed into the optics truss and new data was collected. This data can be seen below in Figure 43.
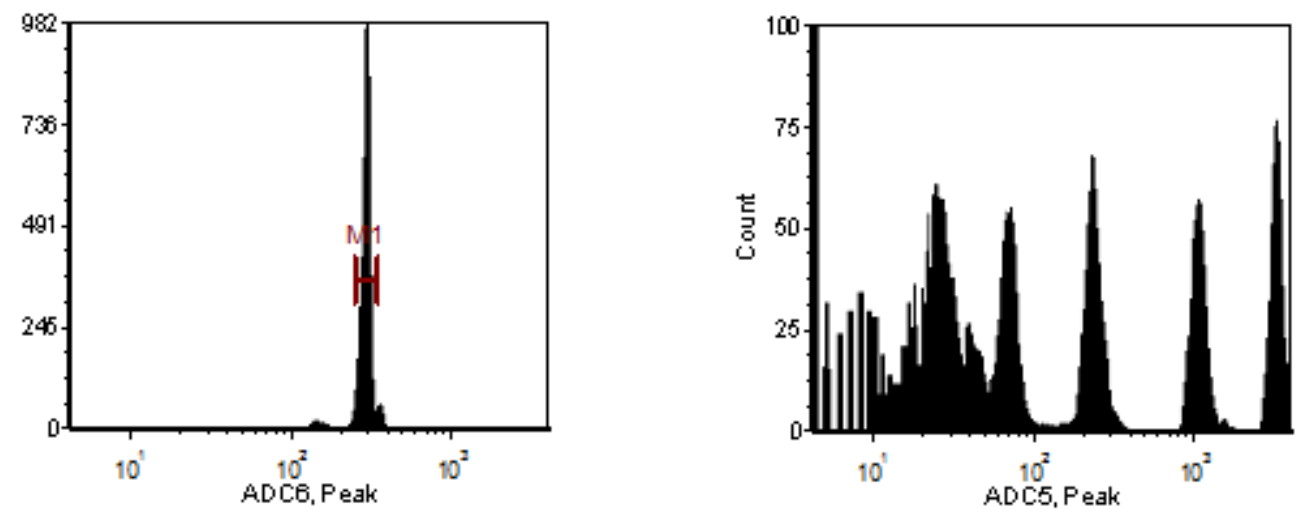

Figure 43. New optics FSC (left) FL1 (right)

This image shows a forward scatter that is roughly the same as the baseline. This is good as nothing in forward scatter was changed allowing for a comparison between the baseline and the new data. As seen in the fluorescent channel on the right the peaks were 
better separated than the baseline. It is clear that the two peaks that were touching in the baseline started to separate slightly from one another. The new dichroics also helped to lower some of the CV's of the peaks. This shows that with the installation of the new dichroics came better data results.

\section{Side Scatter Testing of iteration 2}

Once the fluorescent signals were as optimized as they could be, a new test took place to look at optimization of the laser light traveling through the truss. As mentioned

previously, the light traveling through the truss could not be collimated. This experiment aimed to find an optimal depth for the side scatter lens. The side scatter lens was first positioned in such a way that it gave some data. The experiment recorded data of when the threaded lens was tightened into the flow cell and when it was in a loose position. This would show how large of an effect positioning of the lens had on the fluorescent channels. Two positions were created and the data was recorded. The lens was only tightened a quarter of a turn to see how large a difference this would make in the data. The resulting plots came from FL2 and are seen below in Figure 44 and Figure 45. 


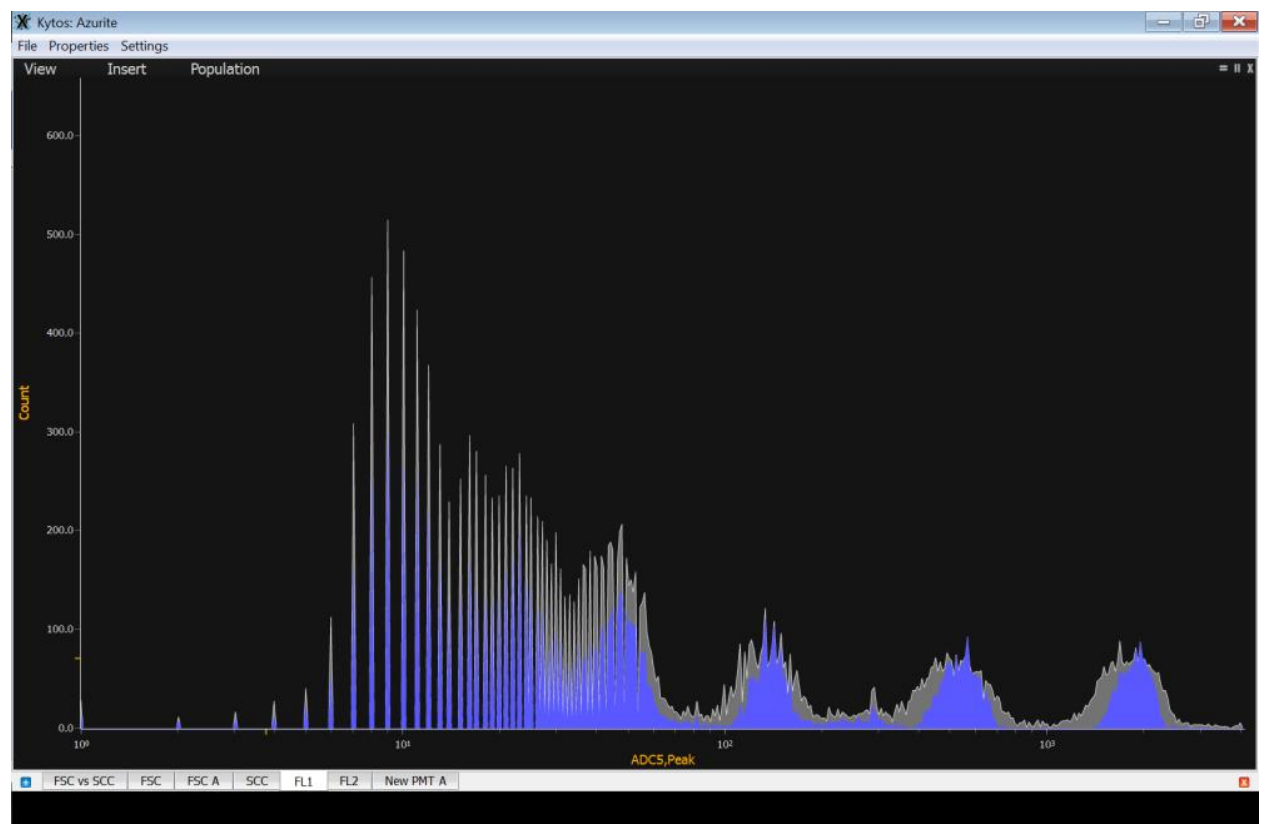

Figure 44. FL2 with a loosened side scatter focus lens

This image was captured off of FL2. In this screen capture the side scatter focal lens was loosened meaning it was positioned farther away from the capillary. There is no way to measure the distance between the capillary and the lens but theoretically moving it farther away from the capillary will cause the focal point to fall between the lens and the capillary. It can be seen that there are roughly 6 peaks in the image with bad separation and bad CVs. 


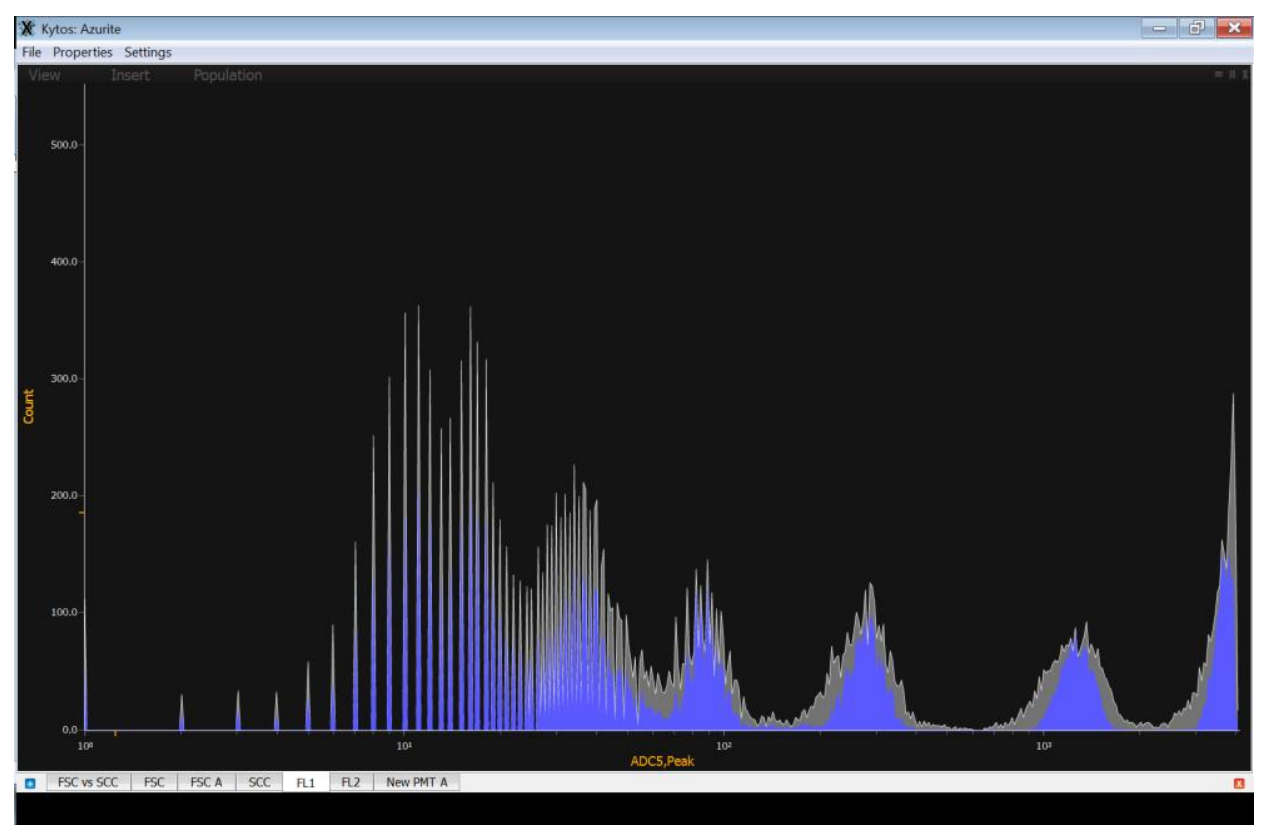

Figure 45. FL2 with a tight side scatter focus lens (Screen capture)

In this image the side scatter lens was tightened, meaning the capillary was closer to the focal length of the lens. It can be seen in this image that again 6 peaks were produced. However, the peaks present in this data are better separated than the peaks produced by the loosened side scatter lens.

By looking at the data it is apparent that a small change in the side scatter lens has a large impact on the signal seen in the fluorescence. When comparing the two it can be seen that the intensity changes drastically causing the signal to fall of the right side of the $\mathrm{X}$ axis. This proved that a unique position was needed for the side scatter mirror to give the best data. 


\section{Redesign 2}

The truss was redesigned to reduce the amount of space that the assembly took. Another benefit of reducing the truss size meant that the light had to travel shorter distances to reach the detectors. By having a shorter light path, small variations in positioning of the optics would have a less dramatic effect on the system. The new truss design can be seen below in Figure 46.

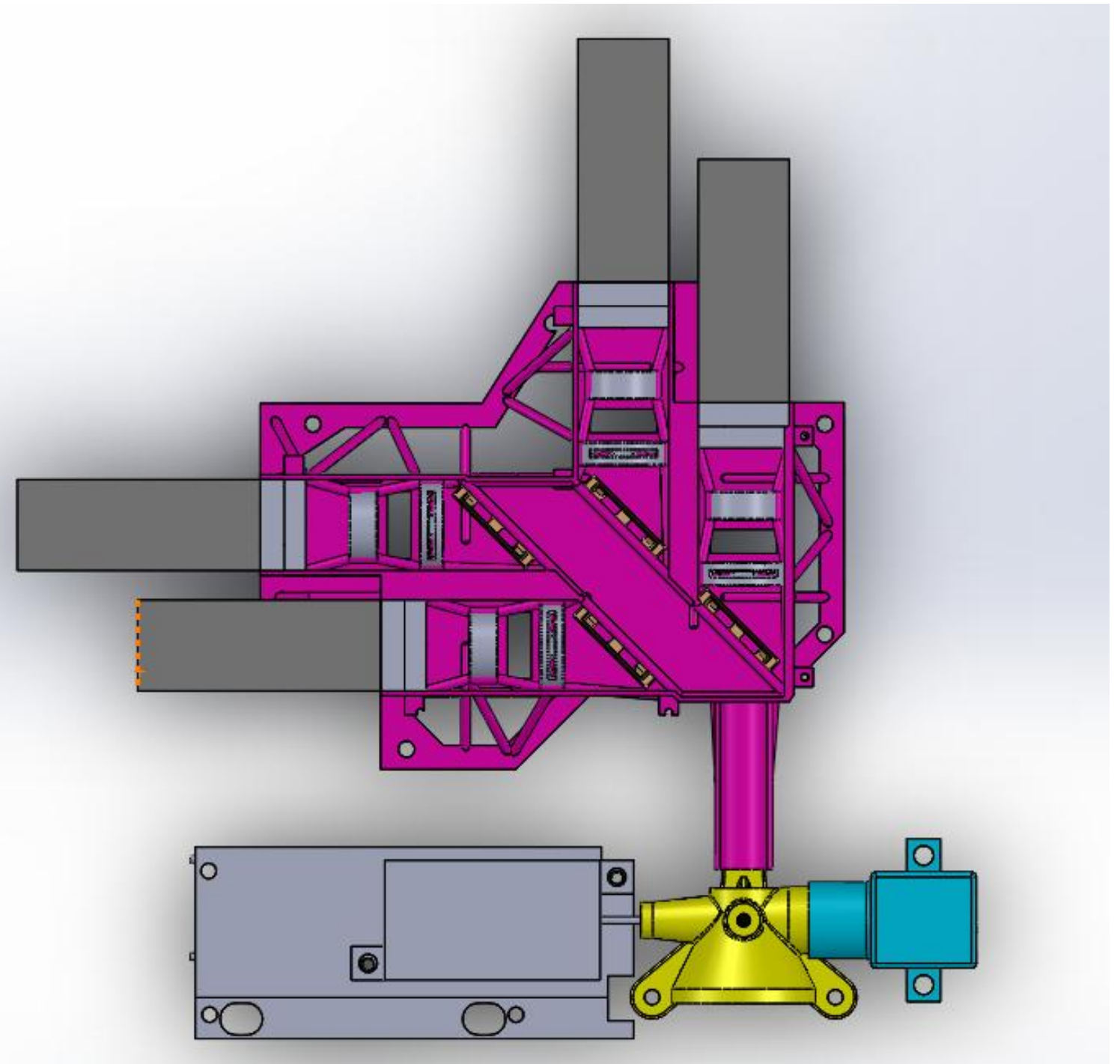

Figure 46. Complete assembly of new alignment optics 
Seen above, the biggest shift was that the PMT's were changed from a horizontal position to a vertical position. This allowed each channel to be closer to the other. The new design effectively reduced the distance the light had to travel by $50 \%$. A tube was also added to reduce the possibility of environmental light entering through the side scatter position. Other than these changes all other properties of the assembly were kept the same this far into the build.

\section{New Laser Alignment}

There were still some issues occurring with the current laser for this build. Another aspect of the rebuild was to see if increasing the intensity of the laser beam would increase the resolution of the 8 peak bead data. The current laser could not change power so a new laser was purchased through Coherent. This laser produced a collimated beam unlike the previous laser. In order to create a beam with a shape that was wanted new optics had to be placed in front of the beam. The new piece of the assembly was created and can be seen below in Figure 47. 


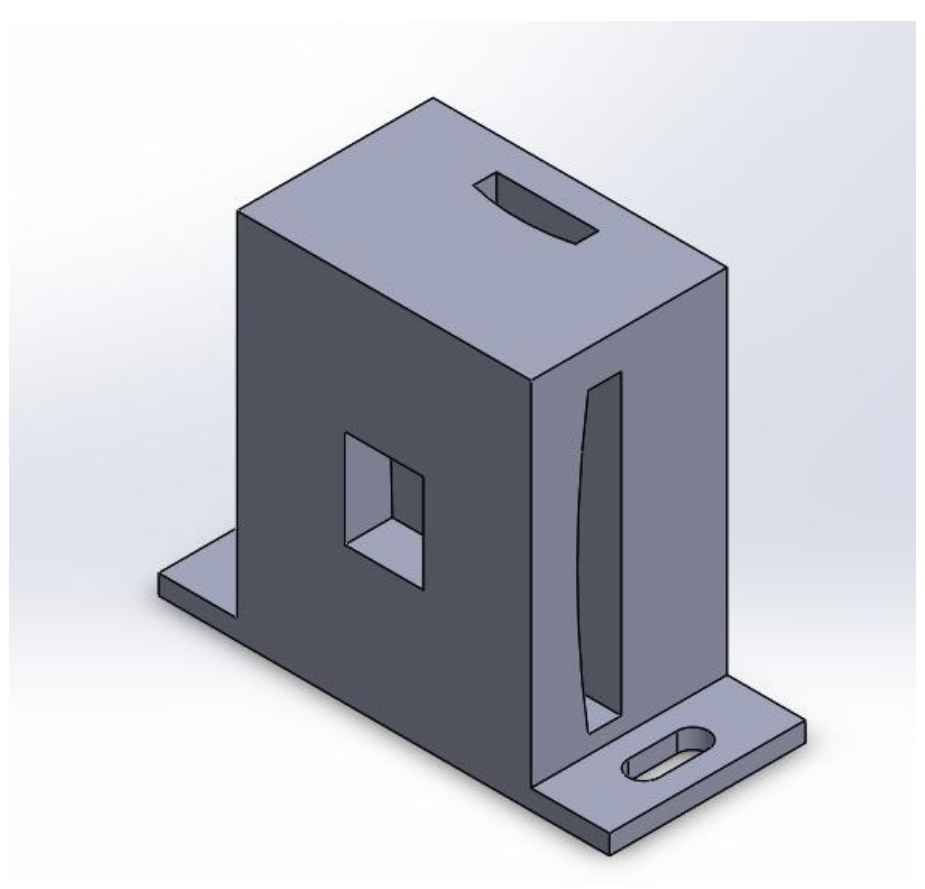

Figure 47. Beam shaper for collimated laser

This new part allowed for two lenses to be inserted into it. One through the top and one through the side. Doing so created a beam with the correct waist line at the capillary. A $200 \mathrm{~mm}$ focal length plano convex lens was placed in through the side to create the necessary Y axis beam waist. Specifications for this lens can be seen in Appendix I.

A $40 \mathrm{~mm}$ focal length was placed through the top to create the necessary $\mathrm{X}$ axis beam waist. Specifications can be seen for this in Appendix J. This component was placed directly in front of the new Coherent laser. A new optics lens also had to be placed into the entrance of the flow cell. This new lens was used to focus the waist line of the beam onto the capillary. This lens was purchased from Edmunds Optics and had a focal length of 8mm. Specs for the piece can be seen in Appendix K. Testing was then done to view the new signal as well as look at the data with different laser powers. The new laser alignment system was then compared to the previous data recording. The focus of this 
experiment was to see if this had an effect on the $\mathrm{CV}$ of forward scatter. Below is an image comparing the data of forward scatter before and after the new laser seen in Figure 48.
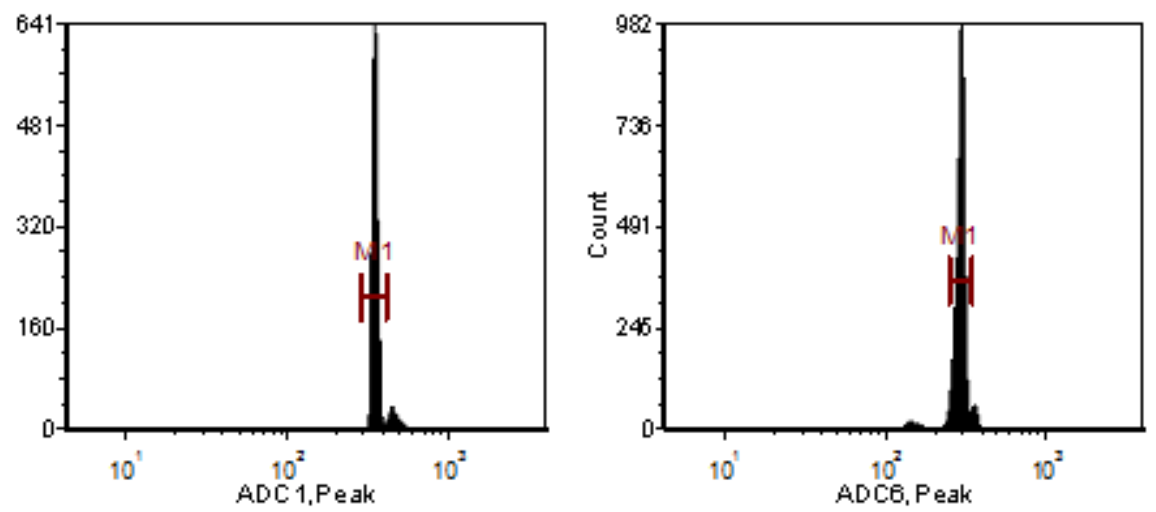

Figure 48. Laser alignment with beam shaper CV 3.94\% (left) vs BSR into capillary CV 5.72\% (right)

The new laser did create a better forward scatter CV. The new laser produced a CV of $3.94 \%$ while the old laser system had a CV 5.72\%. This tighter CV also created tighter CV's in the fluorescent channels as well.

With the new laser, testing to look at the difference in bead signal with different laser powers could be performed. Below are two images comparing a $15 \mathrm{~mW}$ laser, Figure 49, to a $40 \mathrm{~mW}$ laser seen in Figure 50. 


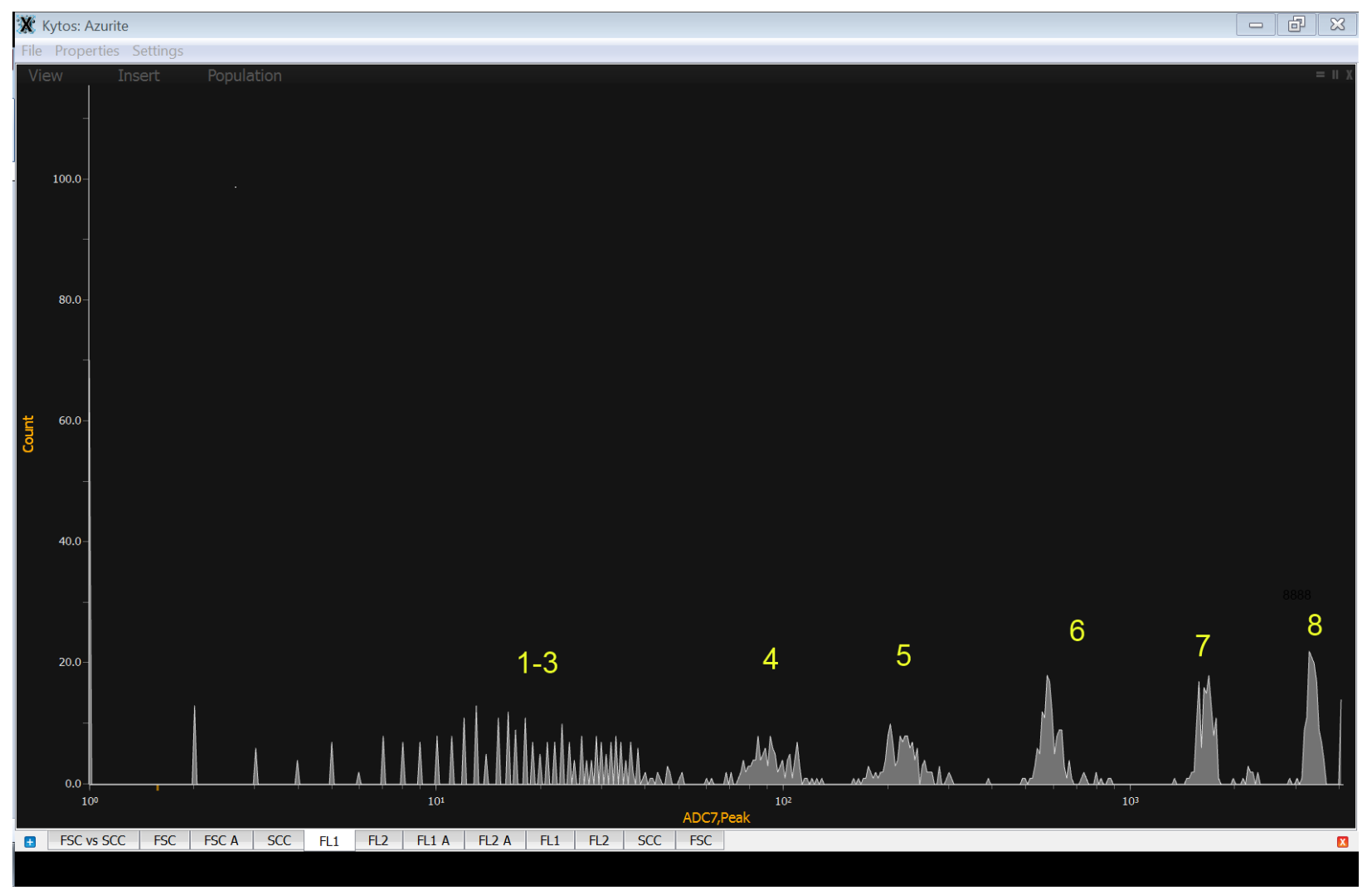

Figure 49. FL2 data resolution with $15 \mathrm{~mW}$ laser

The above image shows data taken from FL2 with a $15 \mathrm{~mW}$ laser. As seen there are 5

distinguishable peaks with the last 3 being bunched together on the low end of the axis.

The laser was then powered on at $40 \mathrm{~mW}$ and another screen capture was taken. 


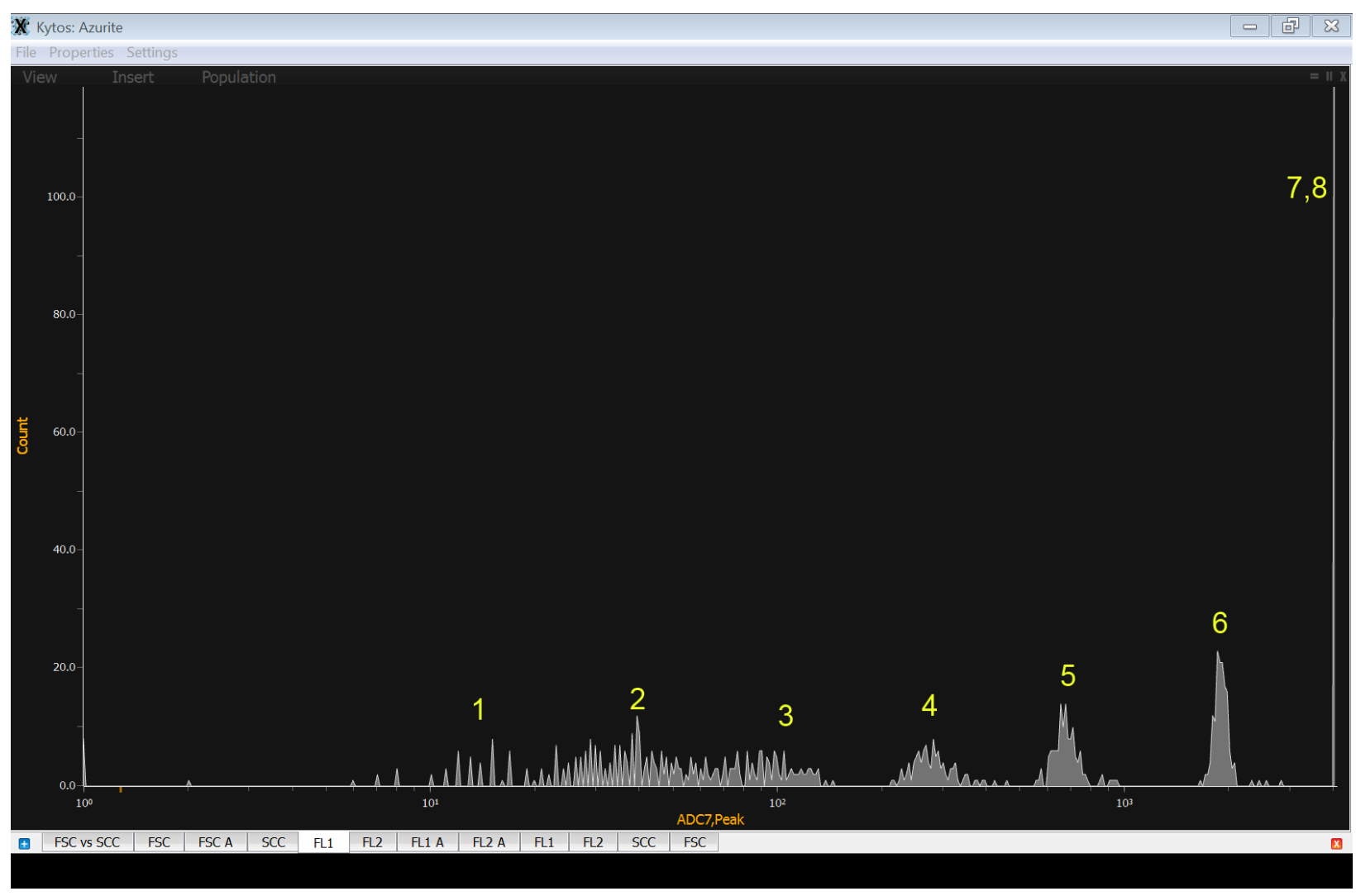

Figure 50. FL2 data resolution with $40 \mathrm{~mW}$ laser

This image shows roughly 6 peaks in the image with the 7 th and 8 th peak off the scale to the right on the FL2 channel.

The positioning of each of the peaks are labeled in the two images above. In Figure 49 the top peaks can be seen but peaks 1-3 are clumped into a pile together toward the bottom. However, in Figure 50 the bottom 3 peaks start to separate and create their own peaks. This does however come with knocking the top peaks of the right of the $\mathrm{X}$ axis. As observed the higher power laser creates a greater intensity of signal. This was successful in showing that all 8 peaks could be resolved with the correct laser power and preamp settings. 


\section{Bluestone Software}

To help with the resolution of the system new software was introduced. This software allowed the system to be moved from a 12-bit system to an 18-bit system. This improvement was to help with peak resolution and give a larger $\mathrm{X}$ axis range on the plots produced. As seen below in Figure 51 this new system allowed for the first complete resolution of 8 peak beads.
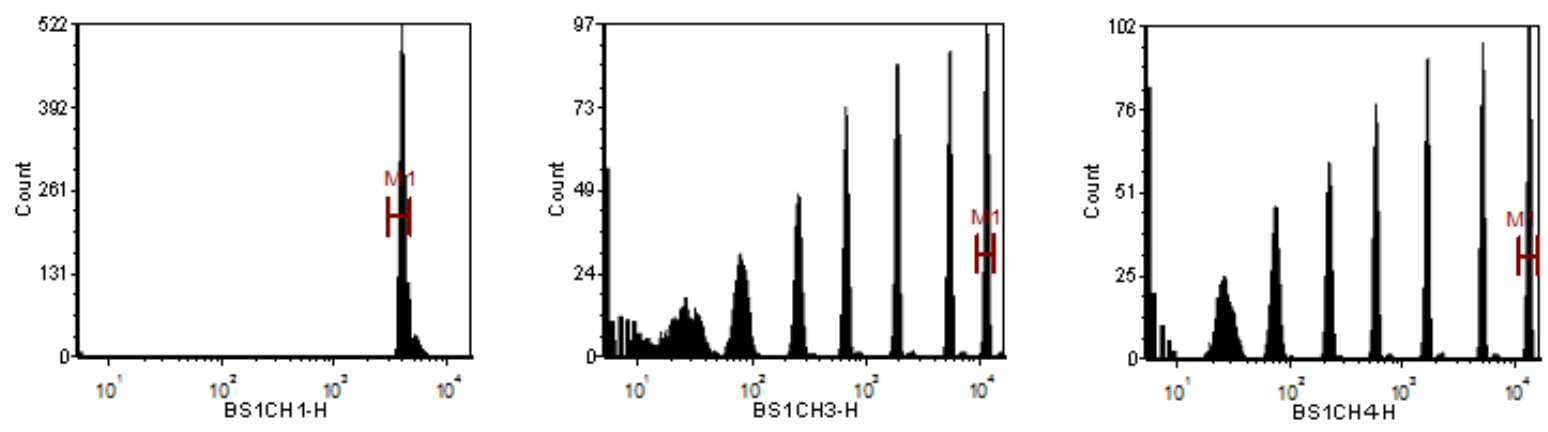

Figure 51. Bluestone data resolution of FSC (left), FL1 (middle), and FL2 (right)

Data was taken from the Bluestone system was able to produce adequate results. All images have low CVs and good separation in the peaks. All 8 peaks can be seen in FL1 and FL2 using this new software.

This system finally gave data that could be presentable and mark the cytometer as a working device. However there was still an issue with the data. All 8 peaks are resolved in the two fluorescent channels above, but this could only be completed by moving the top peak and the bottom peak to the ends of the $\mathrm{X}$ axis. As seen in the images above the bottom peaks can hardly be seen as they are pushed off the plots. 


\section{Refinement of the Assembly}

After implementing changes to the design to produce better data from the assembly a final design was created. The final assembly looks like the image seen below in Figure 52.

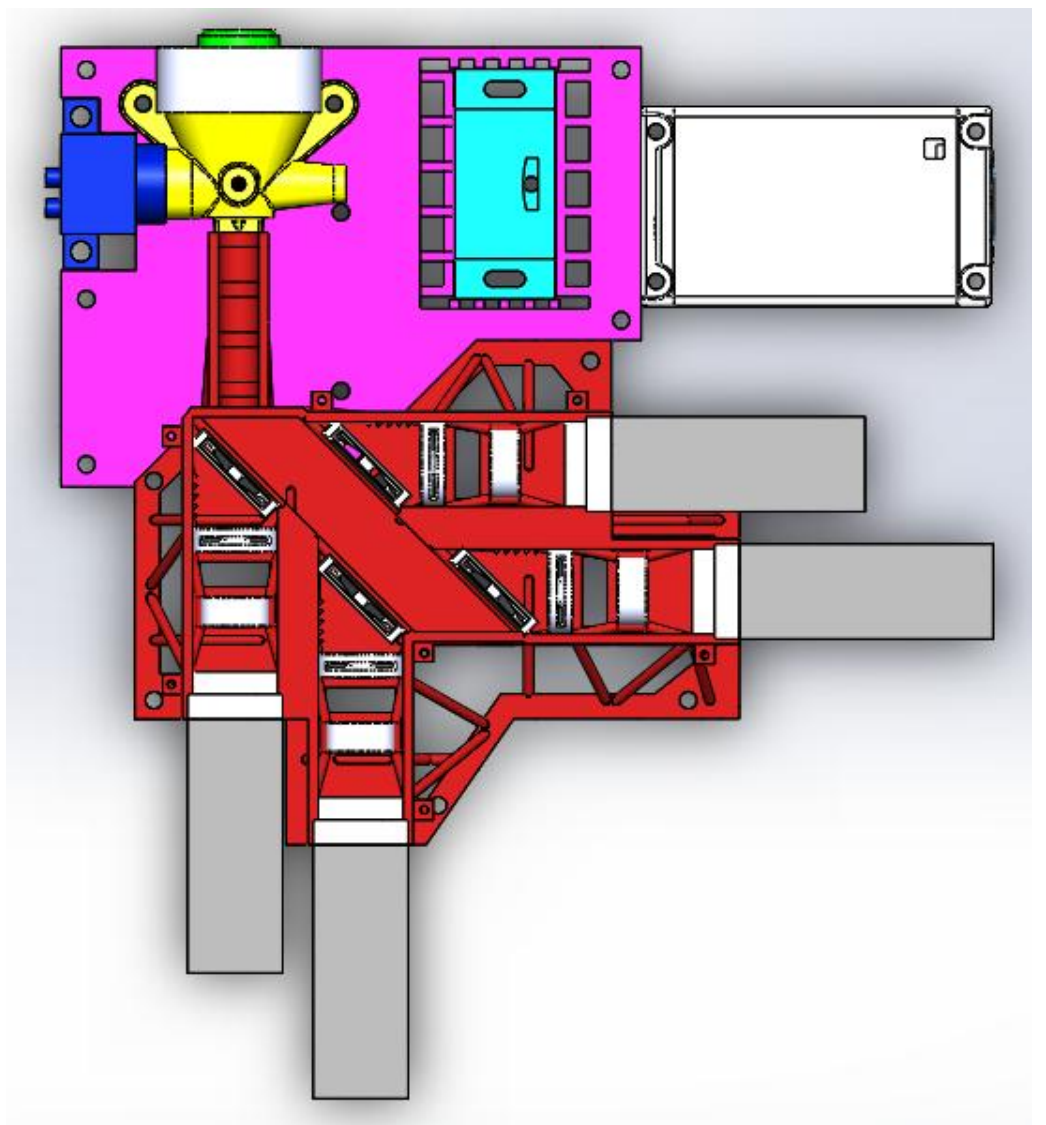

Figure 52. Final optics alignment assembly

The completed assembly was changed for production of the prototypes. These changes aimed to decrease tolerances in the assembly, decrease environmental light noise, and increase data signal. The first addition was the addition of a convex focal mirror added to the flow cell. The assembly looks like that seen below in Figure 53. 


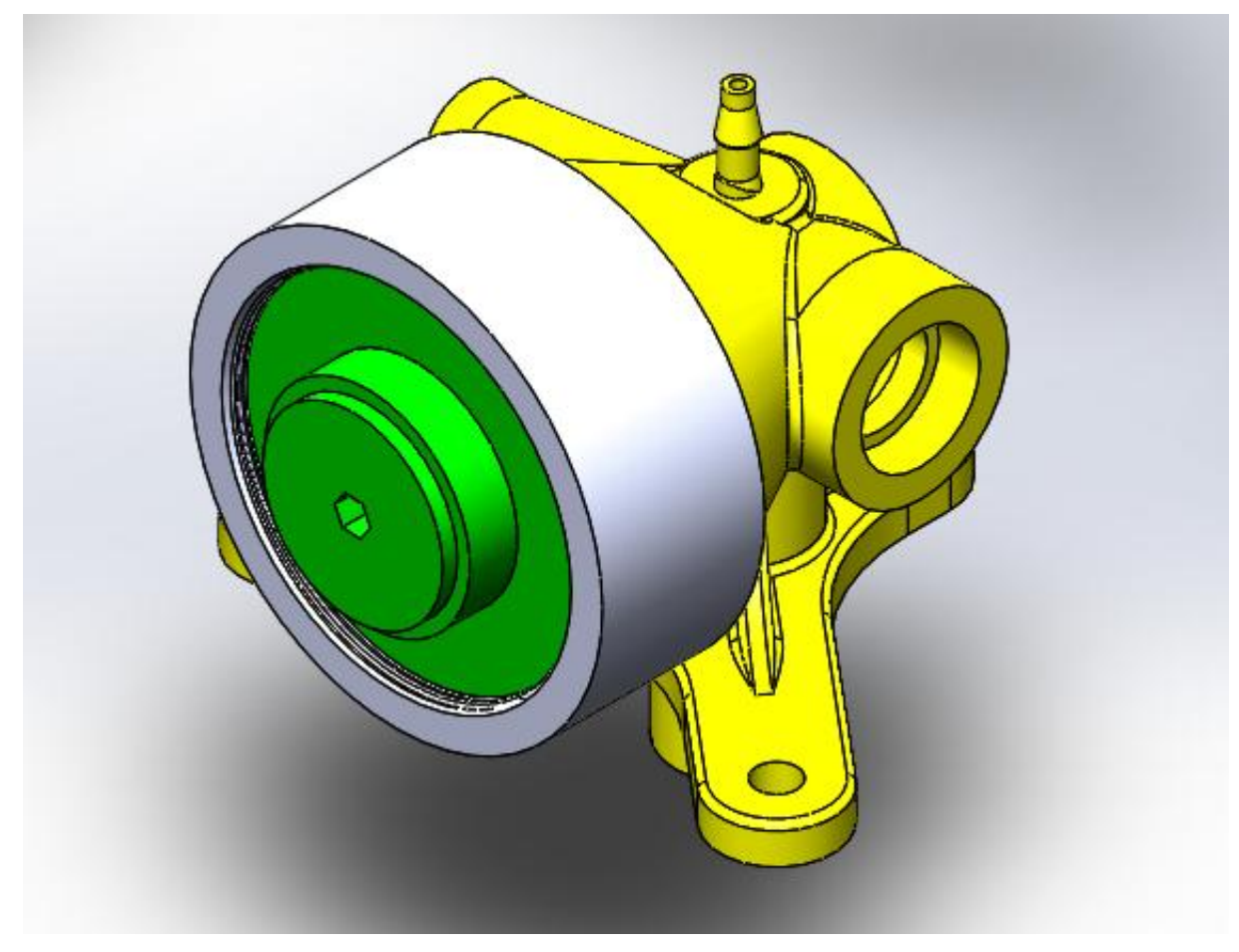

Figure 53. Focal mirror assembly attached to flow cell

A focal mirror was press fit into the green piece and glued in place. The grey piece was then placed over the flow cell and glued in place as well. As both the green and grey piece are threaded this allowed for the focal mirror to be placed at any focal range within the target area. This addition of the mirror helped to add intensity of the signal seen in the fluorescent channels. 
The alignment skeleton was added to help align all pieces in place. The pink skeleton was placed down and all other components were added.

A rough surface was added where light had been previously seen hitting the wall of the truss. The locations can be seen below in Figure 54.

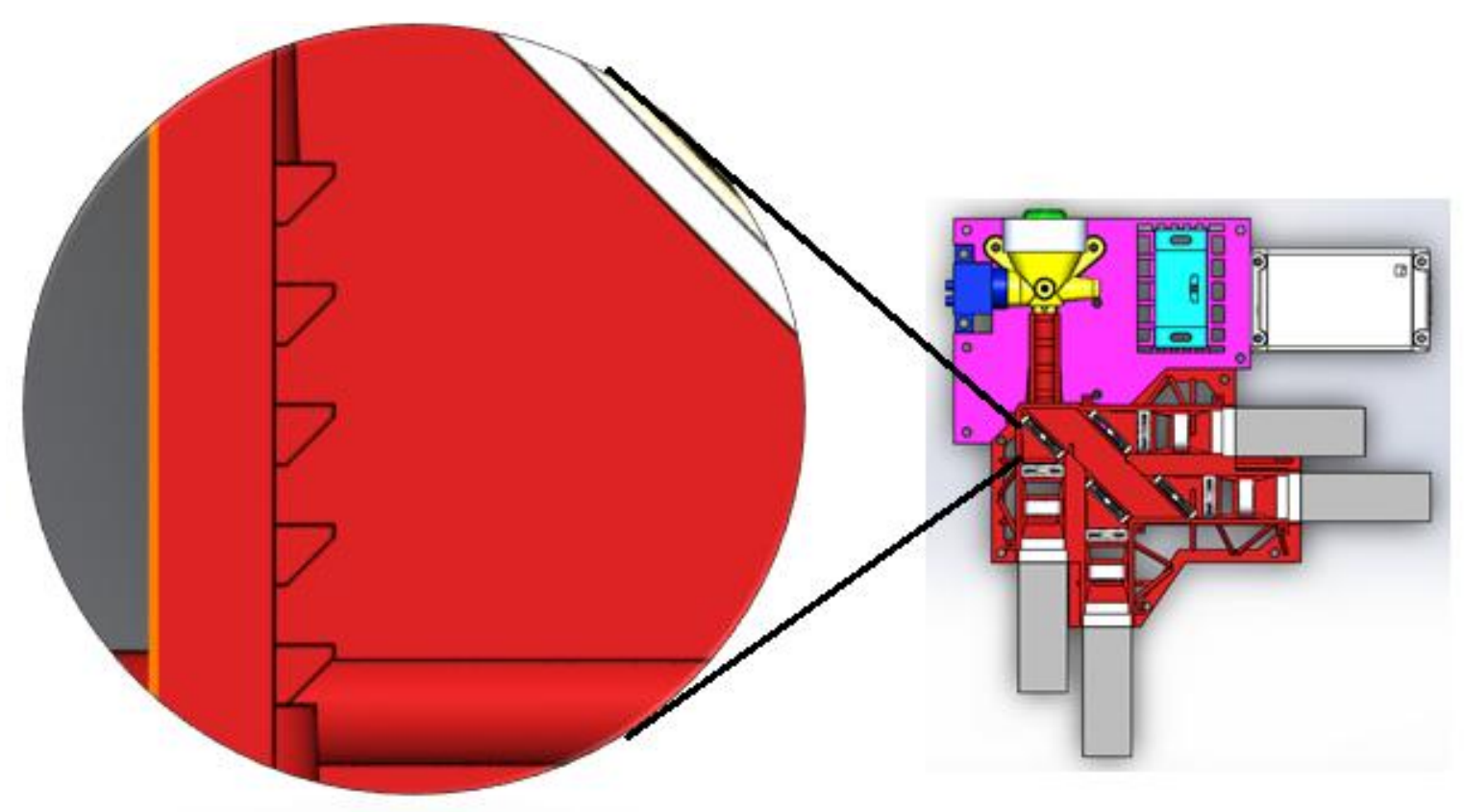

Figure 54. Zoom of light baffles

These surfaces were added to all four channels in the same location. This helped to reduce some of the back reflection that was noted in previous designs. The light will hit the angled surfaces and deflect into the back of the surface in front of it. The thickness of 
all the walls of the truss were also doubled to help reduce environmental light from passing through.

The side scatter entrance tube was also made to reduce environmental light from entering it. As seen below in Figure 55 sharp holes were made for the light to pass through.

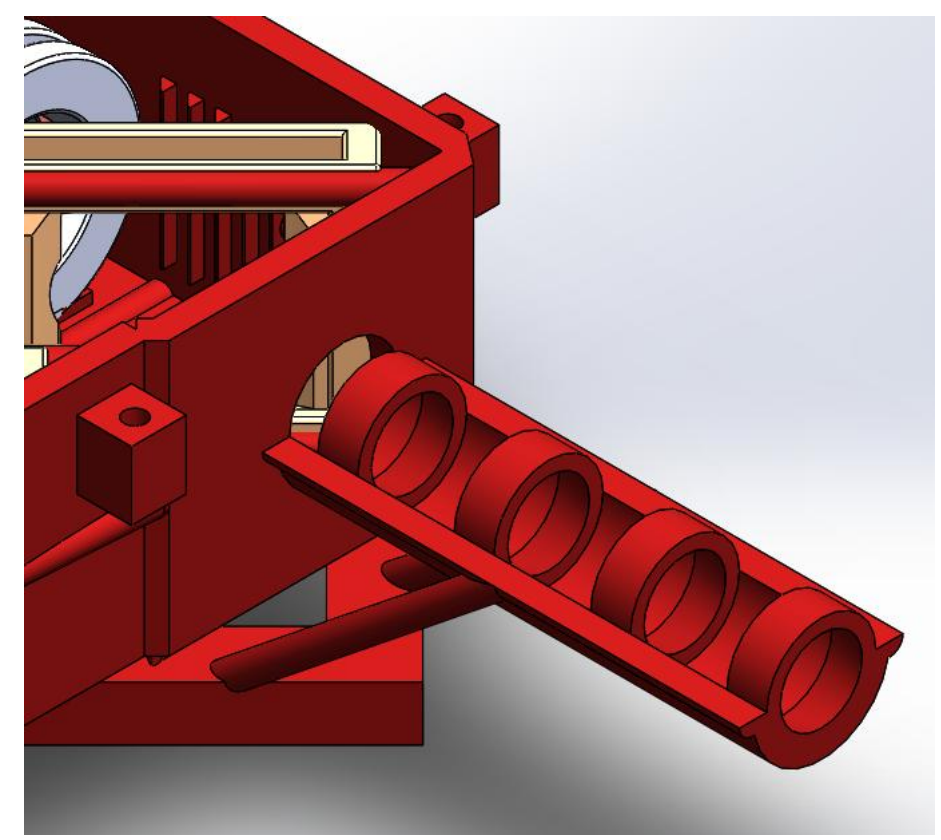

Figure 55. Optics truss light inlet

As seen above the purpose of this feature is to reduce stray light from traveling down the barrel. It is built in much the same way as the pinholes. The front edge is a circle and tapers outward as it travels along the depth.

The lid that goes over the top of the truss is also integrated to close off this opening. Figure 56 shows how the lid integrates with the truss. 


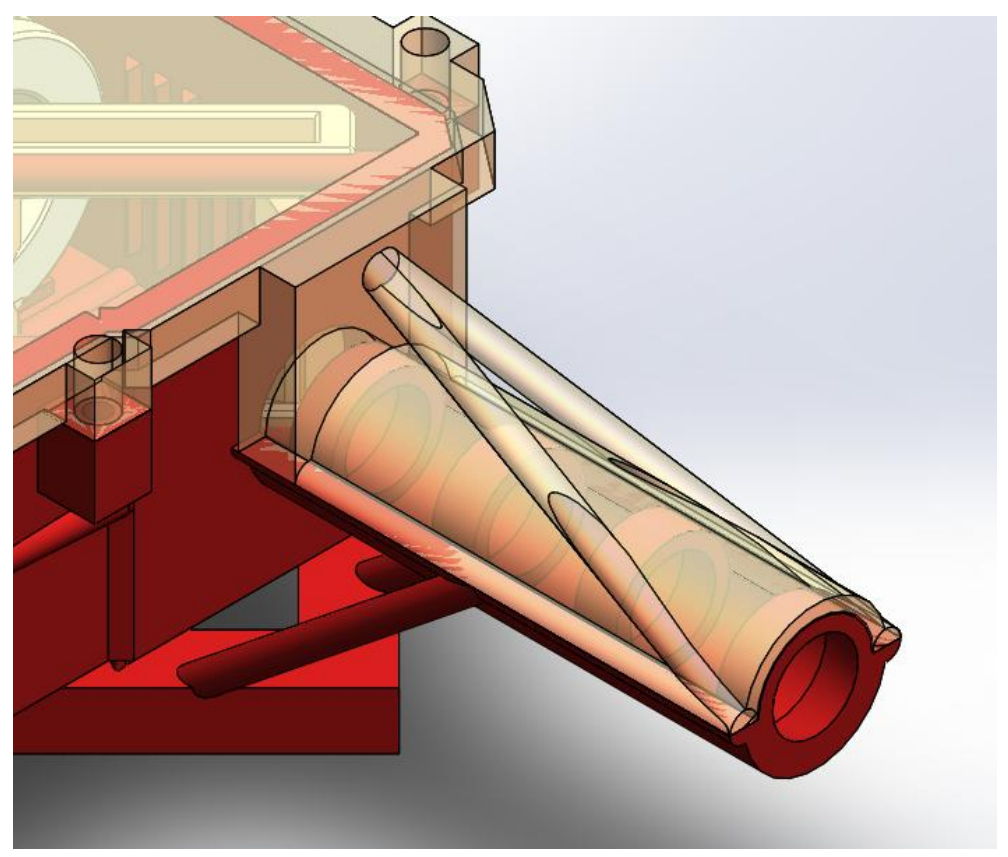

Figure 56. Lid integration with optics truss at light inlet

The lid is used to close off the truss from outside light. This design allows the truss to be easily painted on the inside with a non-reflective coating. This coating ensures that stray light does not continue to bounce around and cause noise in the detectors. 
Complete Final Assembly

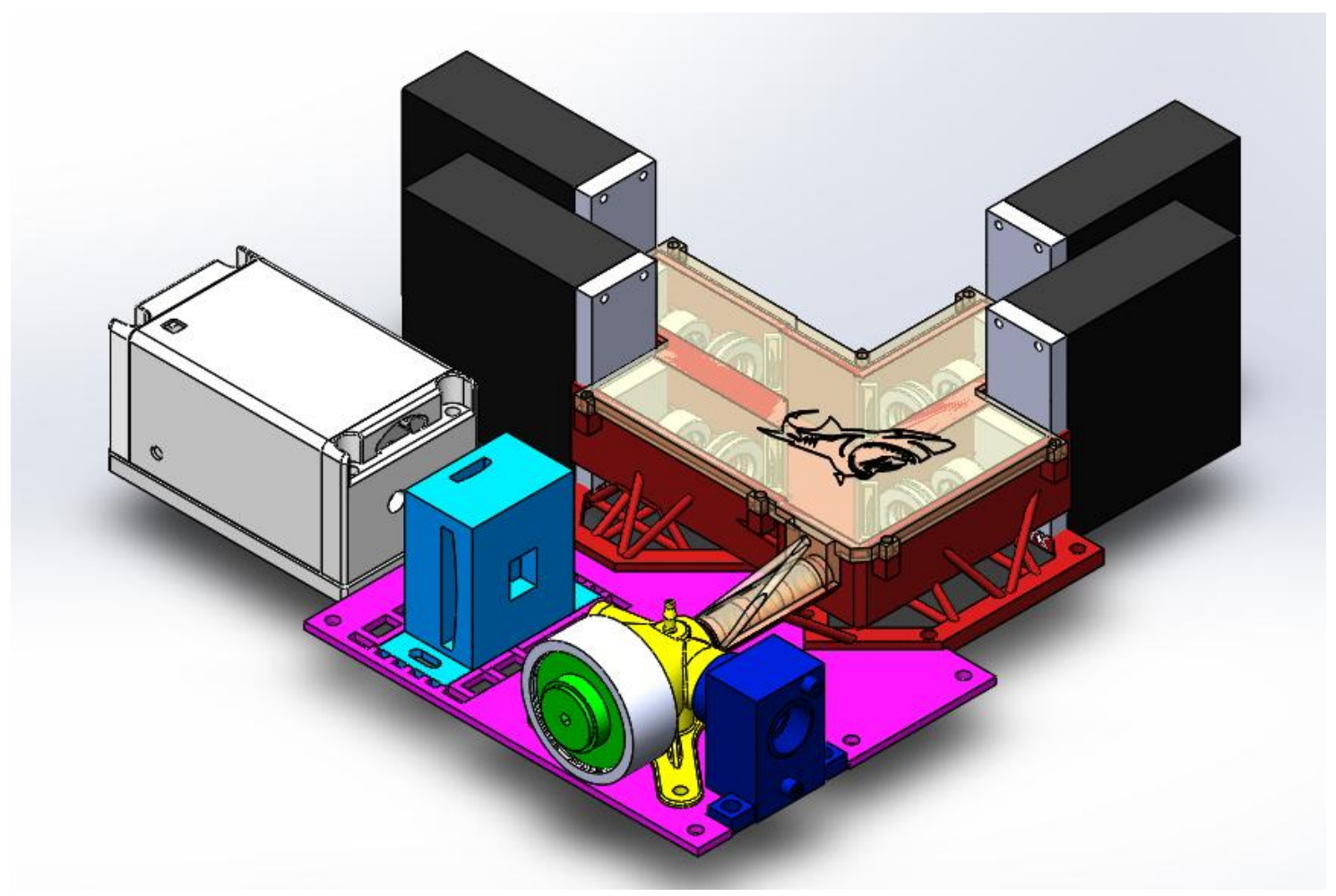

Figure 57. Completed assembly of CytoFlow optics

This is the completed assembly that has gone into the protoypes CytoFlow has produced. It is an isometric view of all components used in the final assembly.

Actual photos of the device can be seen in L.

\section{Conclusion and Recommendations}

The current CytoFlow optics system is operational and is able to collect data as well as other similar devices on the market. Some work will still need to be completed to optimize the device further for manufacturing purposes. 


\section{Future Electronics}

The current electronics for the optics division of CytoFlow involves a preamp as well as an electronic board that the preamp signal runs too. The components are currently separate components that are inserted into the device. It would decrease cost as well as reduce clutter to combine these two components into one board. One caution of doing this would to still ensure that the cable length going from the PMTs to the preamps does not cause signal degradation. Doing this would also allow for software control of the potentiometers found on the preamps. Creation of a single board would also reduce cost for mass manufacturing of the device.

\section{Future Laser}

More work will need to go into the laser design for the CytoFlow device. Current systems use a blue $488 \mathrm{~nm}$ laser in conjunction with a red $560 \mathrm{~nm}$ laser. A laser that has both these wavelengths has been produced by Blue Sky Research for integration within Cytoflow's device. Current work on this topic is geared toward making an electronic board that can drive both the lasers. The lasers will be modulated so that the system is able to pick up and sort the signal from the two different colors. Work on the software side of the device is also being implemented so that the correct laser signal is sorted into the correct charts.

\section{Future Manufacturing}

If CytoFlow decides to move away from mass 3D printing of the truss work will need to be done to change the design. Currently there is no other way to create the structure of the truss. To produce a truss that is machined or molded the truss will need to be broken into separate pieces. Doing this would allow the truss to be operated on in all axis. This 
would most likely involve removing a section of the fluorescent channels that is then assembled to create the optics assembly.

\section{Future of the Device}

The finished product will be a device that can be used for clinical applications specific for what the company is looking for. The device will be able to run blood samples and be tuned to what one expects the outcomes to be. There is also room for modification where seen fit. Some companies will want different laser wavelengths as well as multiple wavelengths. These systems will be used to analyze the cells at question and help to improve the world.

This device is aimed at use for clinical as well as research applications. The hopes are to be able to use the device to look at all biological cells that current cytometers are able to view. A specific area where this device is applicable is for AIDS testing in Africa. The device is portable so it can be transported by vehicle and could run off a car battery. Allowing researchers to track AIDS development in Africa. The device is also making way with a company who looks at oil purities. By running this companies chemical in oil concentrations it will be able to look at the fluorescence and show the purities of the oil. In all this device is to be used as a diagnostic tool in areas where high throughput and statistical means are needed. 


\section{BIBLIOGRAPHY}

[1] "What Is Flow Cytometry?" News-Medical.net. 11 May 2010. Web. 5 Mar. 2015.

[2] Brown, Michael, and Carl Wittwer. "Flow Cytometry: Principles and Clinical Applications in Hematology." Flow Cytometry: Principles and Clinical Applications in Hematology. Clinical Chemistry, 1 Aug. 2000. Web. 5 Mar. 2015.

[3] "Evolution Flow: The Historical Background of Flow Cytometry." Bitesize Bio. Bitesize Bio, 28 Jan. 2013. Web. 19 Feb. 2015.

[4] "BD Accuri C6." BD Biosciences Accuri C6 Personal Flow Cytometer. BD Biosciences. Web. 19 Feb. 2015.

[5] "Data Analysis: What does a histogram tell me?." Home. Oregon State, n.d. Web. 10 May 2014. <http://www.unsolvedmysteries.oregonstate.edu/flow_07>.

[6] David L. Jaye, Cissy M. Geigerman, Ross E. Fuller, Adil Akyildiz, Charles A.

Parkos, Direct fluorochrome labeling of phage display library clones for studying binding specificities: applications in flow cytometry and fluorescence microscopy, Journal of Immunological Methods, Volume 295, Issues 1-2, December 2004, Pages 119-127, ISSN 0022-1759, http://dx.doi.org/10.1016/j.jim.2004.09.011.

[7] Filter of Flow Cytometry. Digital image. Semrock. N.p., n.d. Web. < http://www.semrock.com/Data/Sites/1/semrockimages/drawings/flowcytometry_500.jpg>.

[8] Bair, Nathan. "Cytometery Discussion." Personal interview. 1 Feb. 2014.

[9] "Dichroic Mirrors." Encyclopedia of Laser Physics and Technology. RP Photonics. Web. 5 Mar. 2015.

[10] "Flow Cytometry Guide." Abcam. Web. 19 Feb. 2015. <http://docs.abcam.com/pdf/protocols/Introduction_to_flow_cytometry_May_10. pdf $>$.

[11] "Photomultiplier Tubes Basics and Applications." Hamamatsu. Web. 19 Feb. 2015. <https://www.hamamatsu.com/resources/pdf/etd/PMT_handbook_v3aE.pdf>.

[12] So, Peter. "Biological Engineering II: Instrumentation and Measurement." MIT, Cambridge. 1 Oct. 2006. Class Lecture.

[13] J. Vives-Rego, P. Lebaron, G. Nebe-von Caron, Current and future applications of flow cytometry in aquatic microbiology, FEMS Microbiology Reviews, 
Volume 24, Issue 4, October 2000, Pages 429-448, ISSN 0168-6445, http://dx.doi.org/10.1016/S0168- 6445(00)00033-4. 


\section{A. Forward Scatter Focus Lens}

Attached in this appendix is the lens that is used in the flow cell to collimate the beam upon exiting the flow cell. This lens is used to make sure that a collimated beam is passed onto the forward scatter detector as well as ensure the beam blocker is only blocking what it needs to. 


\section{TECHefer $9 \mathrm{~mm}$ Dia. $\mathbf{x} 9 \mathrm{~mm} \mathrm{FL}_{\text {, }} \mathrm{MgF}_{2}$ Coated, Double-Convex Lens}

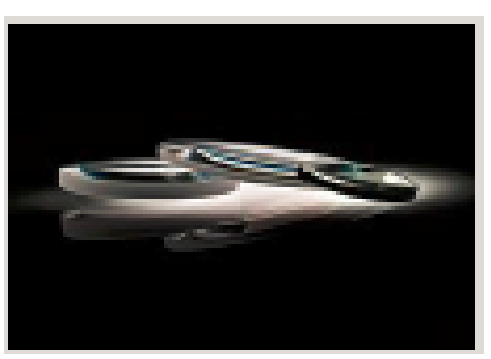

strek Mo. H32-193

hwallability: Fander:

430.00

1. - E for wo wo anch.

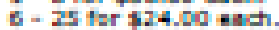

\section{SPECIRICATIOUS}

\begin{tabular}{|c|c|}
\hline 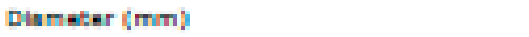 & 7.m \\
\hline Diumeter Tolemea |mm] & $+0.00 y-10$ \\
\hline Cleir Aperture Cu (mm) & D.1 \\
\hline 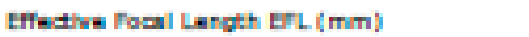 & P. \\
\hline 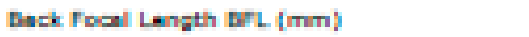 & T.9o \\
\hline Focal Length Tolerance ith j & $\$ 1$. \\
\hline Fadiug $A_{1}=-A_{j}|m|$ & 11.10 \\
\hline 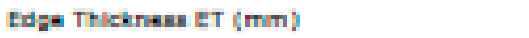 & 1.e0 \\
\hline Center Thichnew [T Imin] & $3+5$ \\
\hline Carter Tt|denesa Tolernan [mm] & LII \\
\hline Cartaring [aromirulad & $3-5$ \\
\hline 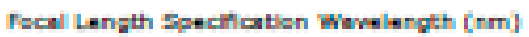 & ExT.E \\
\hline Surtuce Quality & $40-30$ \\
\hline Denel & Fratotke terelias neded \\
\hline Subutate & HEIS \\
\hline C=ating & $\mathrm{MpF}_{\mathrm{I}}$ \\
\hline Canting Spadhenthar & 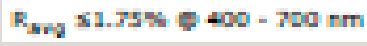 \\
\hline Typlad Enamy Deralty Lmit & $101 \mathrm{~m}^{2}$ q $19 \mathrm{~nm}, 10 \mathrm{ra}$ \\
\hline TrFe & Double-Conven L-1: \\
\hline tit & 1 \\
\hline MLmerical heertiore MA. & as \\
\hline Bwrelerglh Panpe lum] & $5+-87$ \\
\hline Farts & c \\
\hline
\end{tabular}

TECHYICML I4RORMATIOK

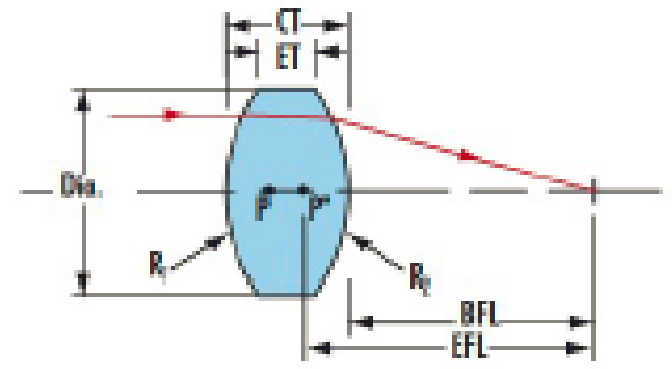

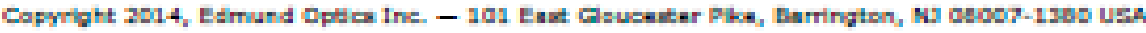

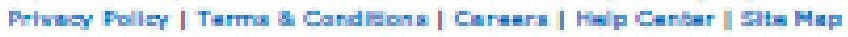


B. Side Scatter Focus Lens

This Appendix contains information on the side scatter lens. This lens is used to focus and collimate the light that exits out of the flow cell orthogonal to the forward scatter. This lens is used as a means of focusing light that is coming off the capillary and passing it to the side scatter and fluorescent channels. 


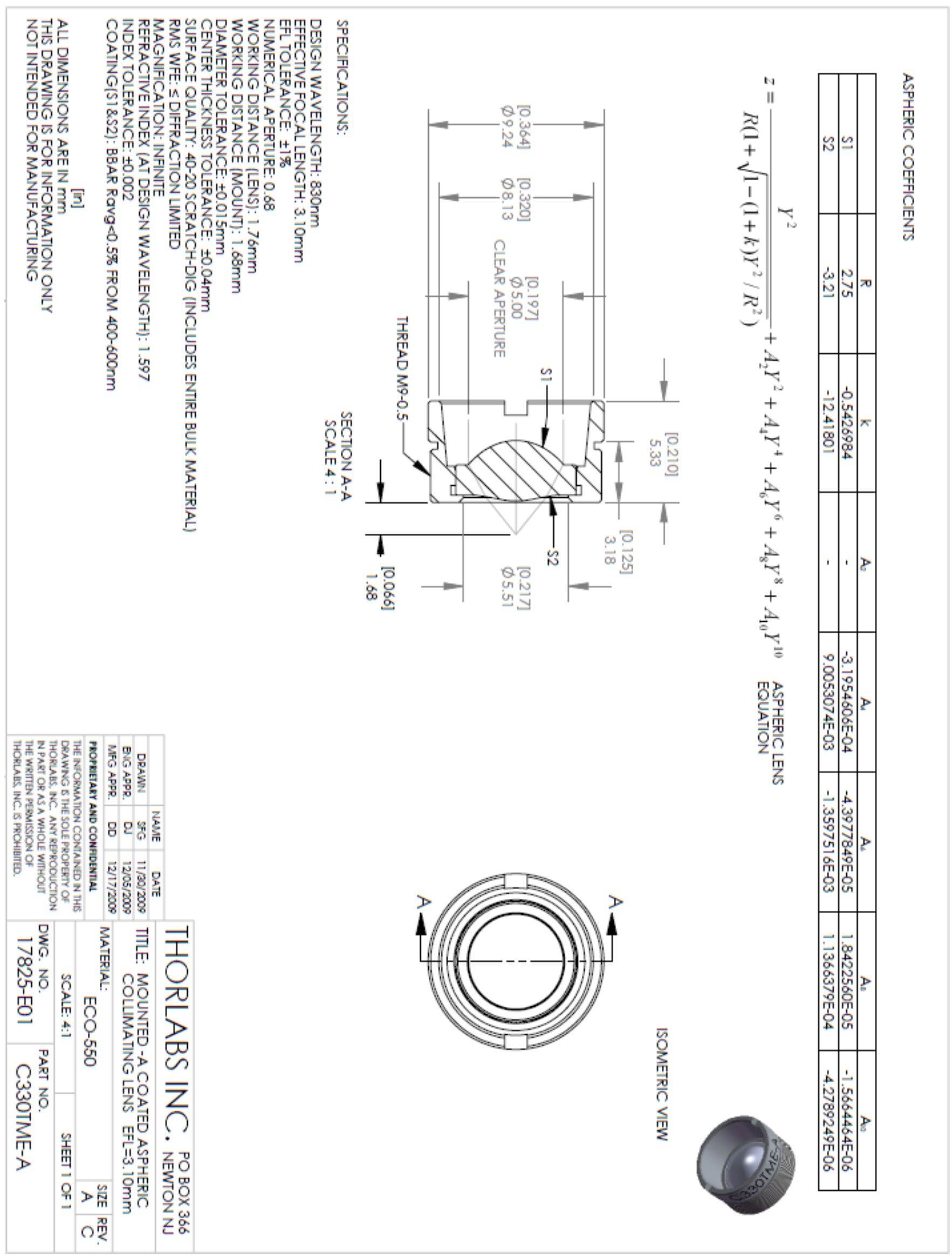




\section{Fluorescence Dichroic Mirrors}

This Appendix contains the specification of the dichroic mirrors used in the optics truss. These dichroic mirrors were purchased from CHROMA. The images below show the CHROMA specs for transmission. 


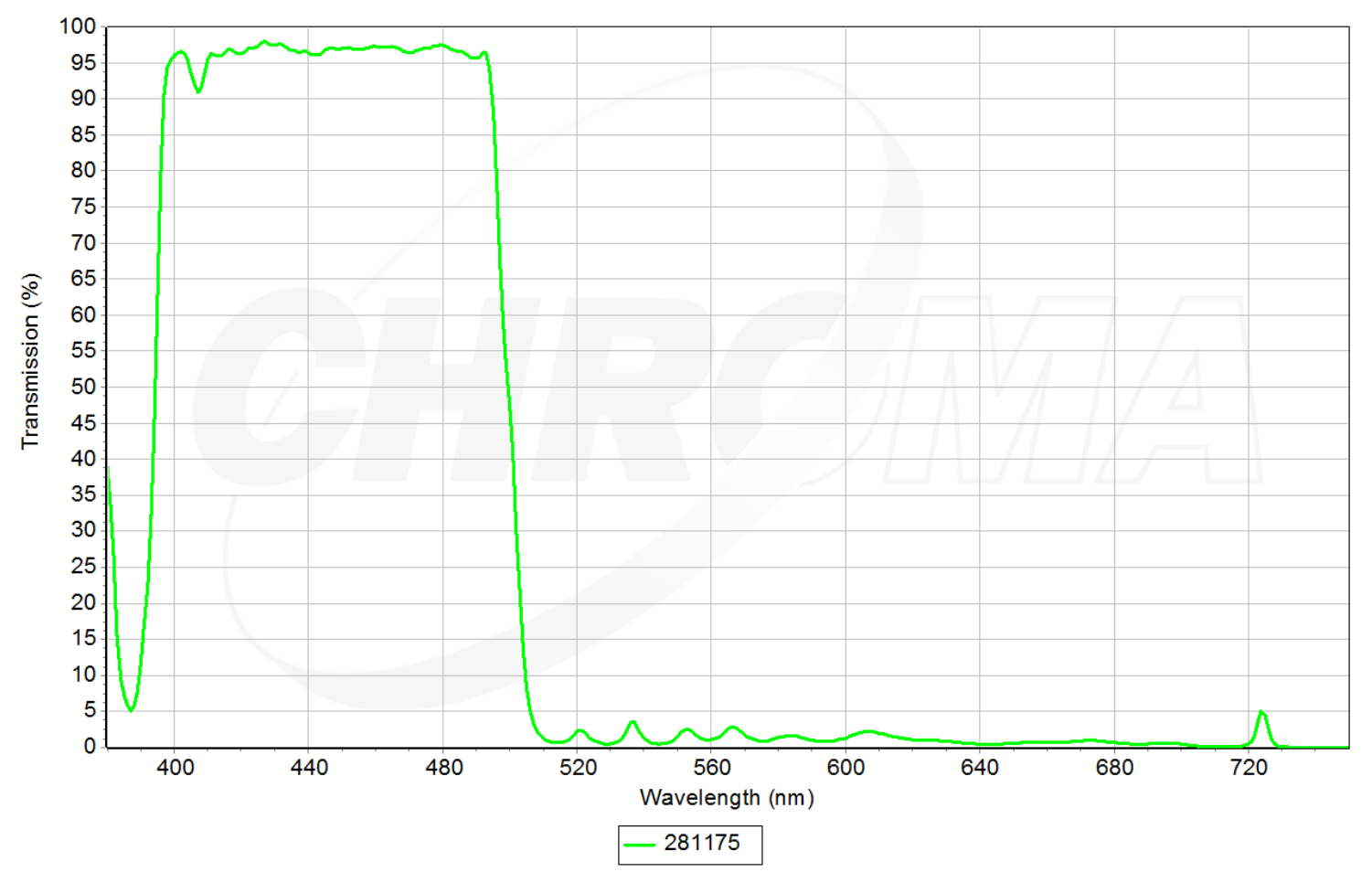

T500 Shortpass Dichroic Mirror

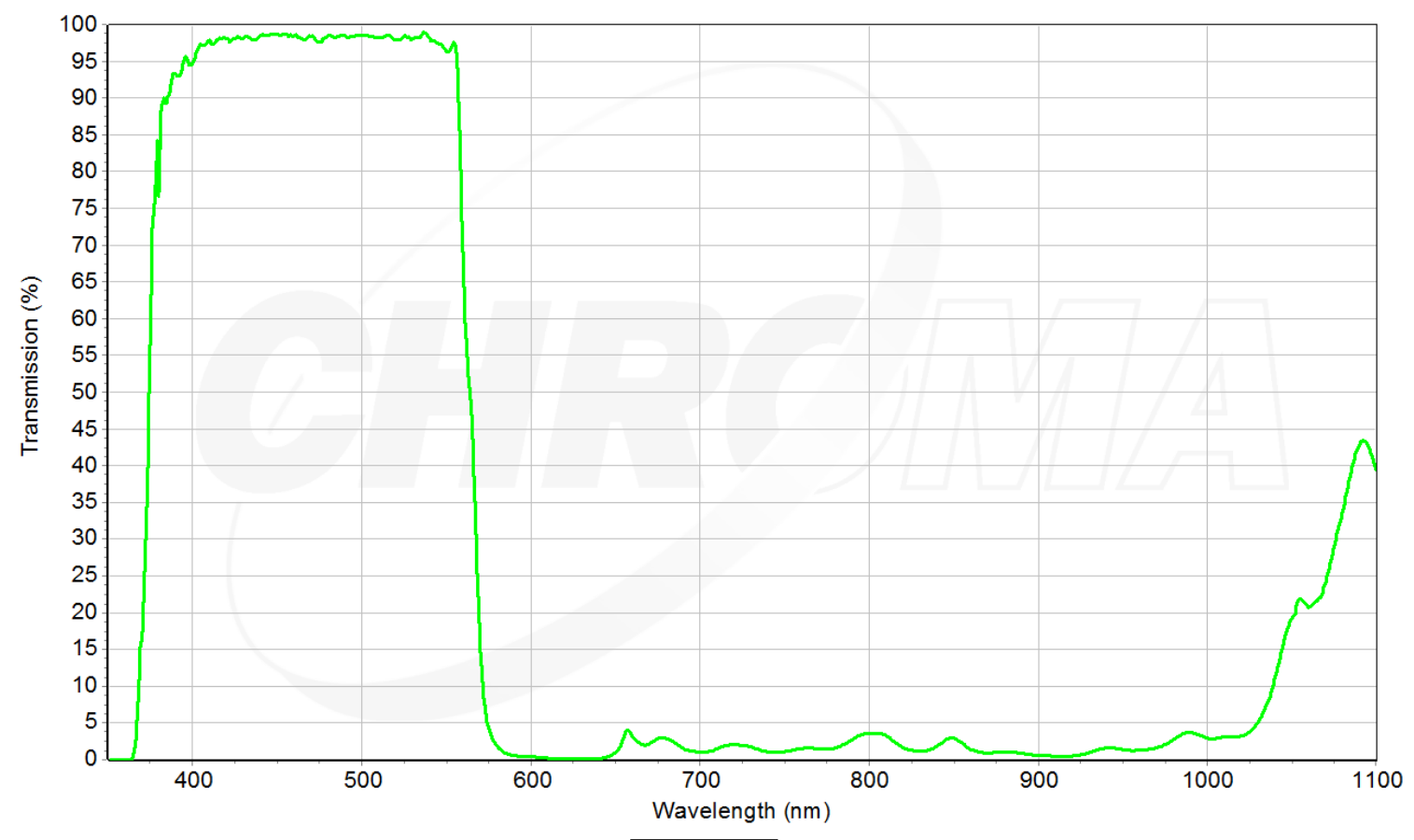

$-281171$

T565 Shortpass Dichroic Mirror 


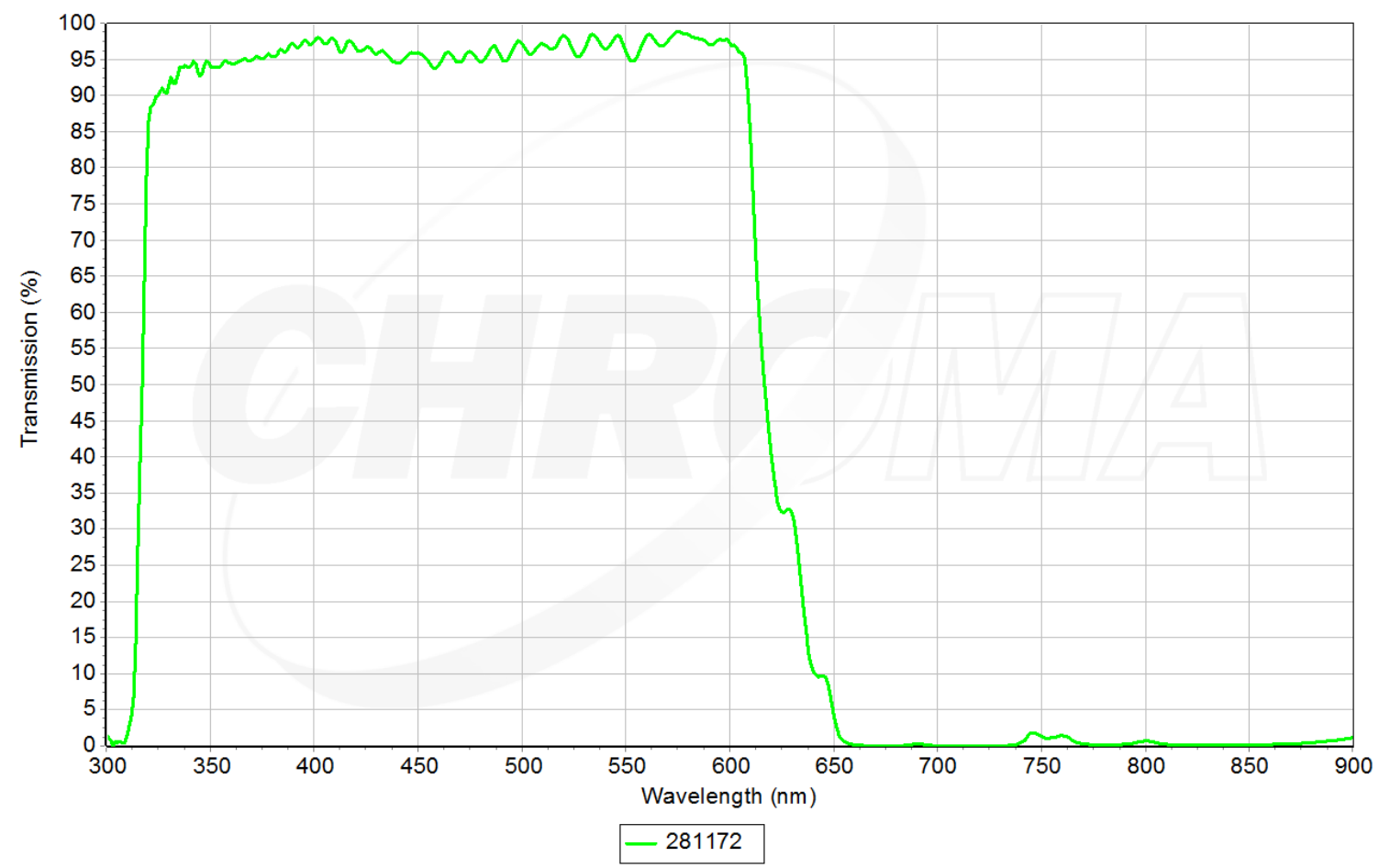

T615 Shortpass Dichroic Mirror

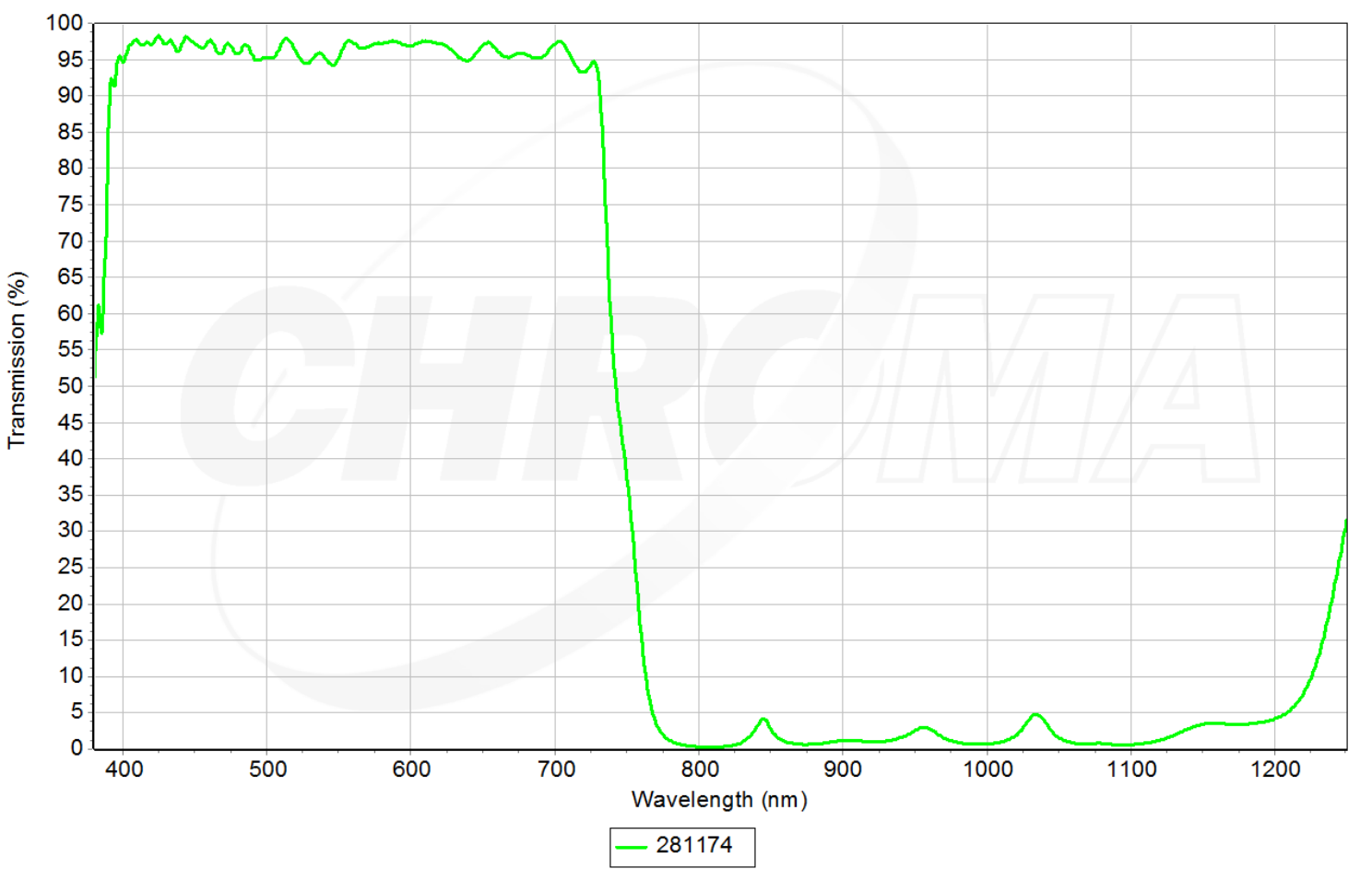

T745 Shortpass Dichroic Mirror 


\section{Fluorescence Filters}

This Appendix contains the specifications of the filters used in the optics truss. These filters were purchased from CHROMA. 


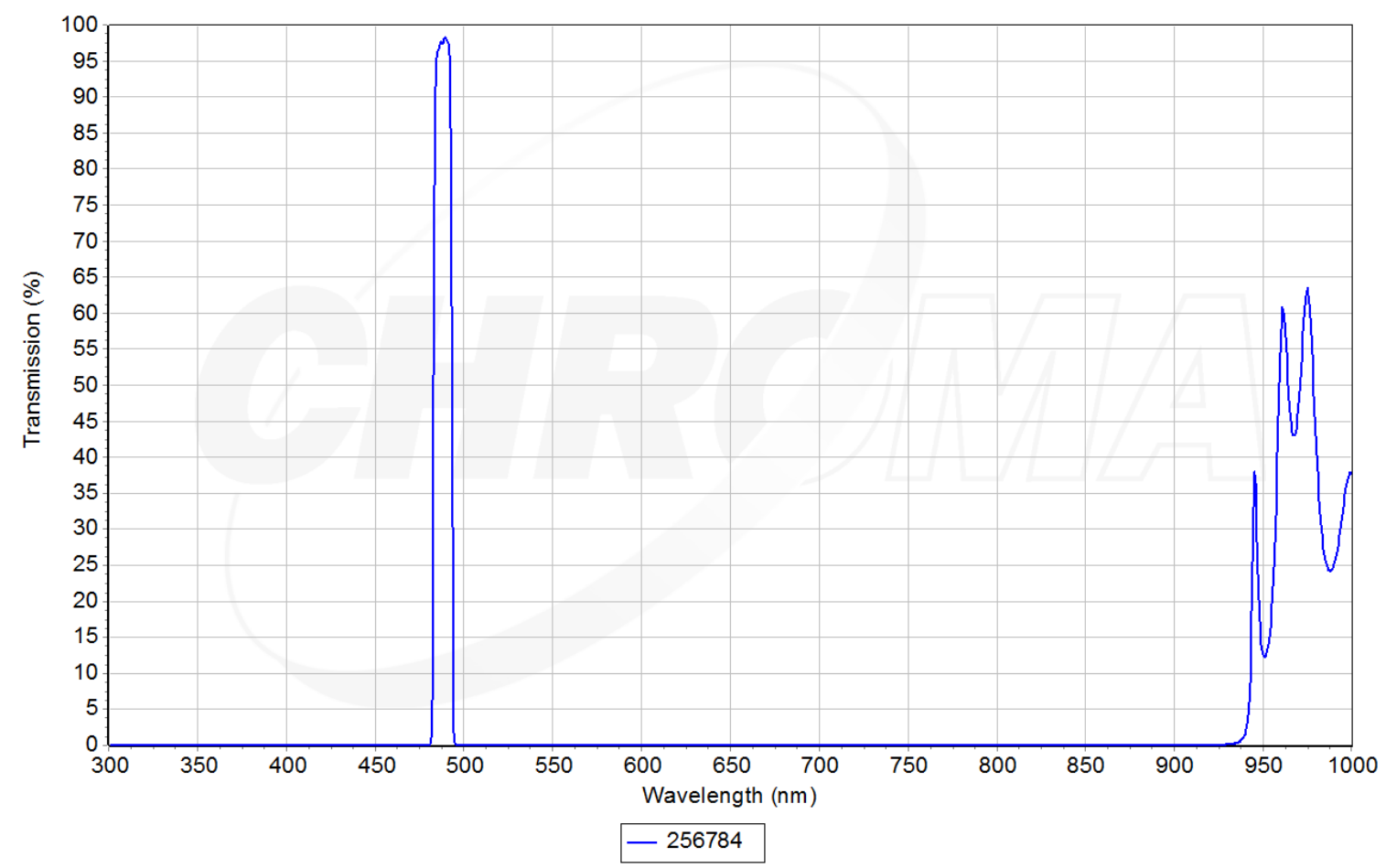

ET488/10 Bandpass Filter

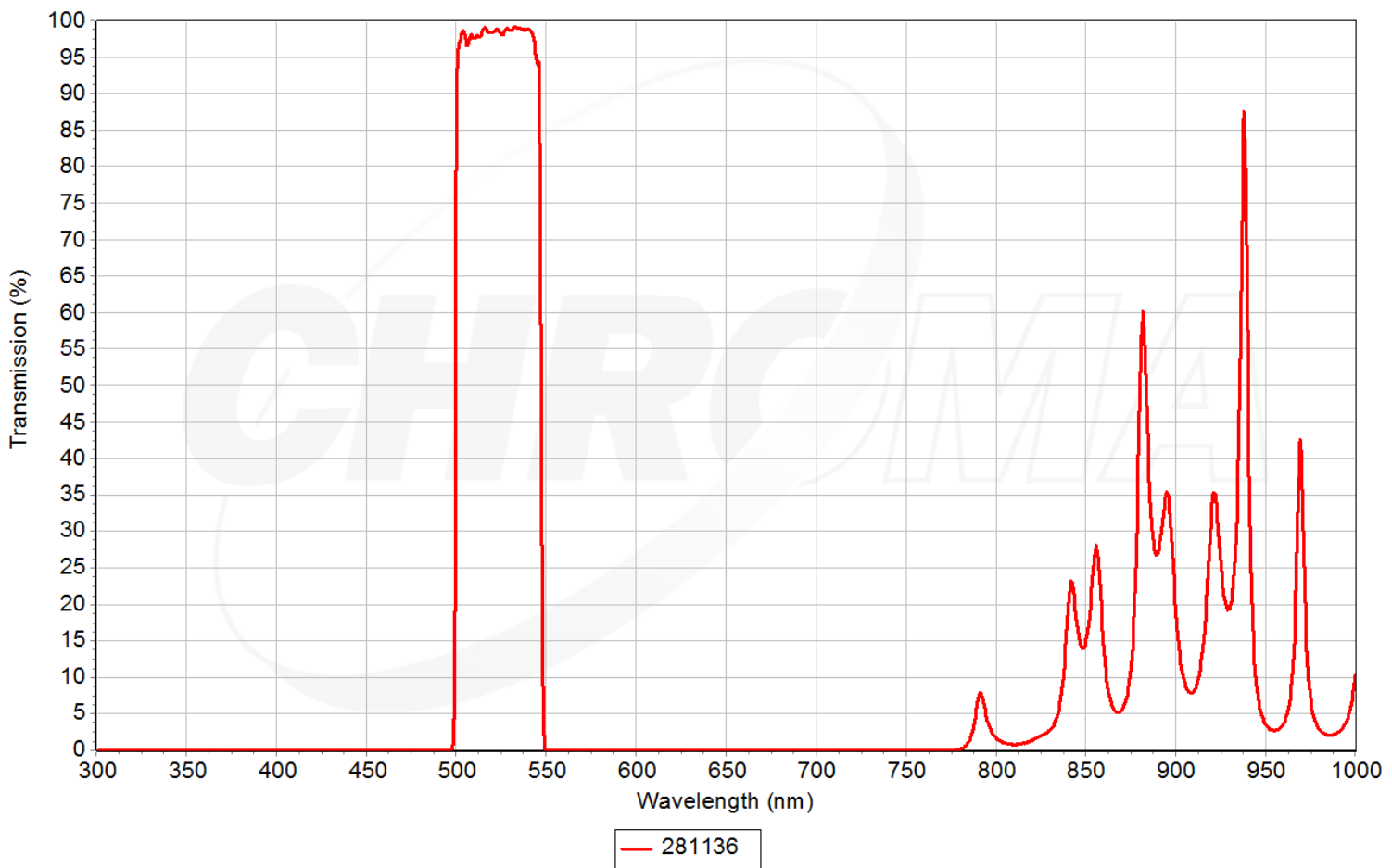

ET 525/50 Bandpass Filter 


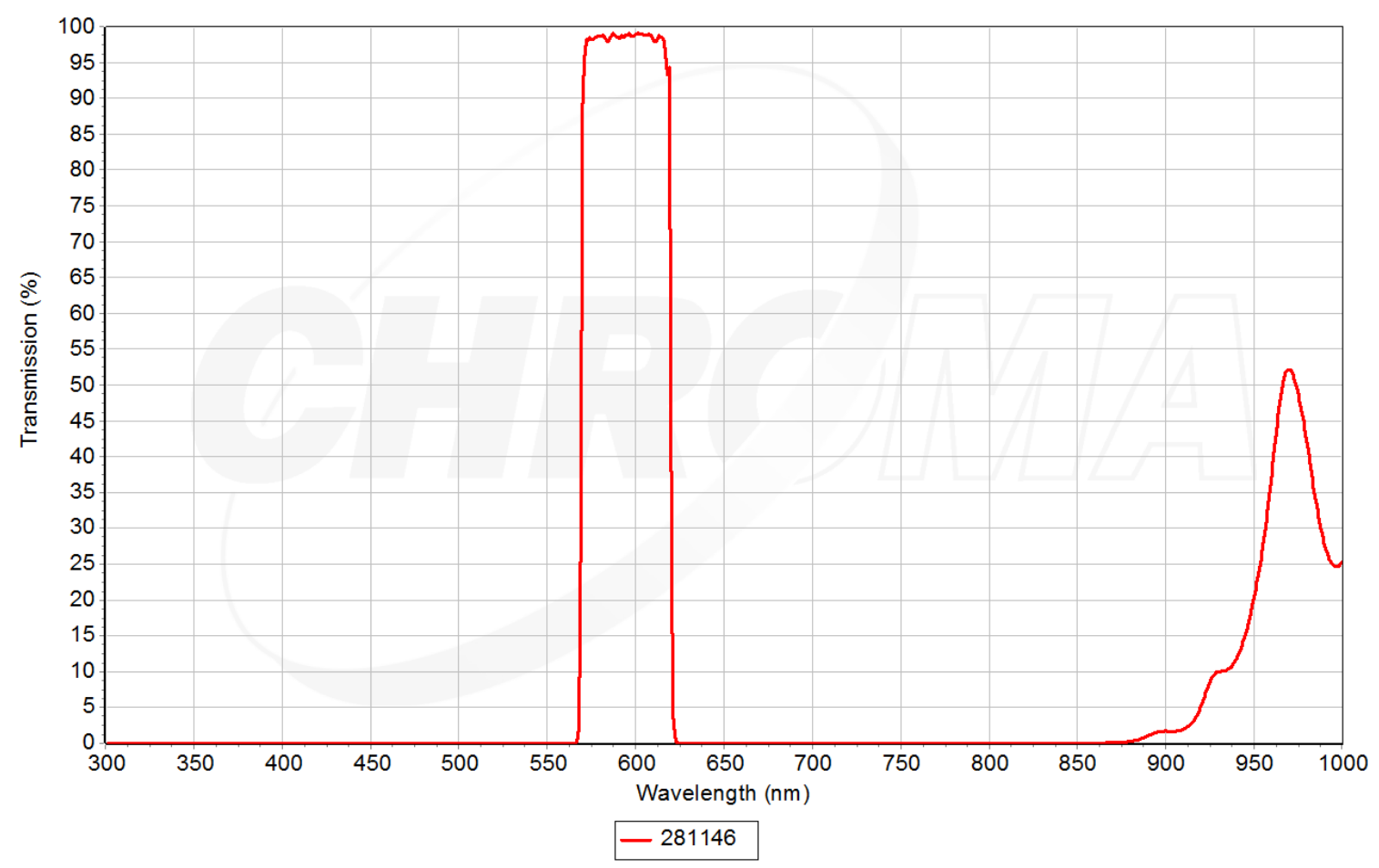

ET595/50 Bandpass Filter

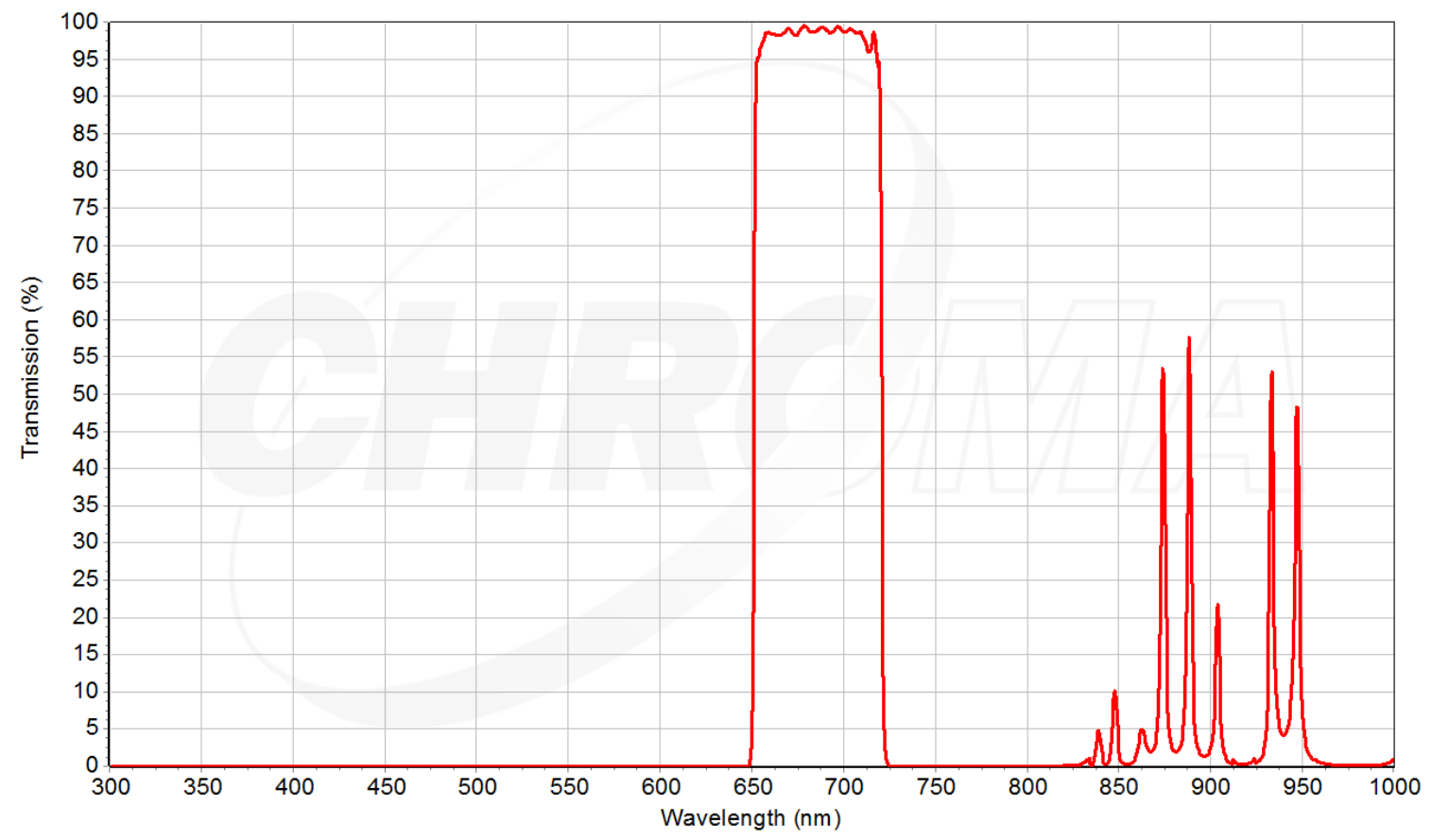

$-281195$

ET685/70 Bandpass Filter 


\section{E. Optics Truss Focus Lens}

This Appendix contains specifications of the focal lenses used in the optical truss. These lenses are used to focus light traveling through the side scatter and fluorescent channels into the PMT. These can be used to adjust where the focal point of the light in the channel is, increasing and decreasing signal seen by the detector. 

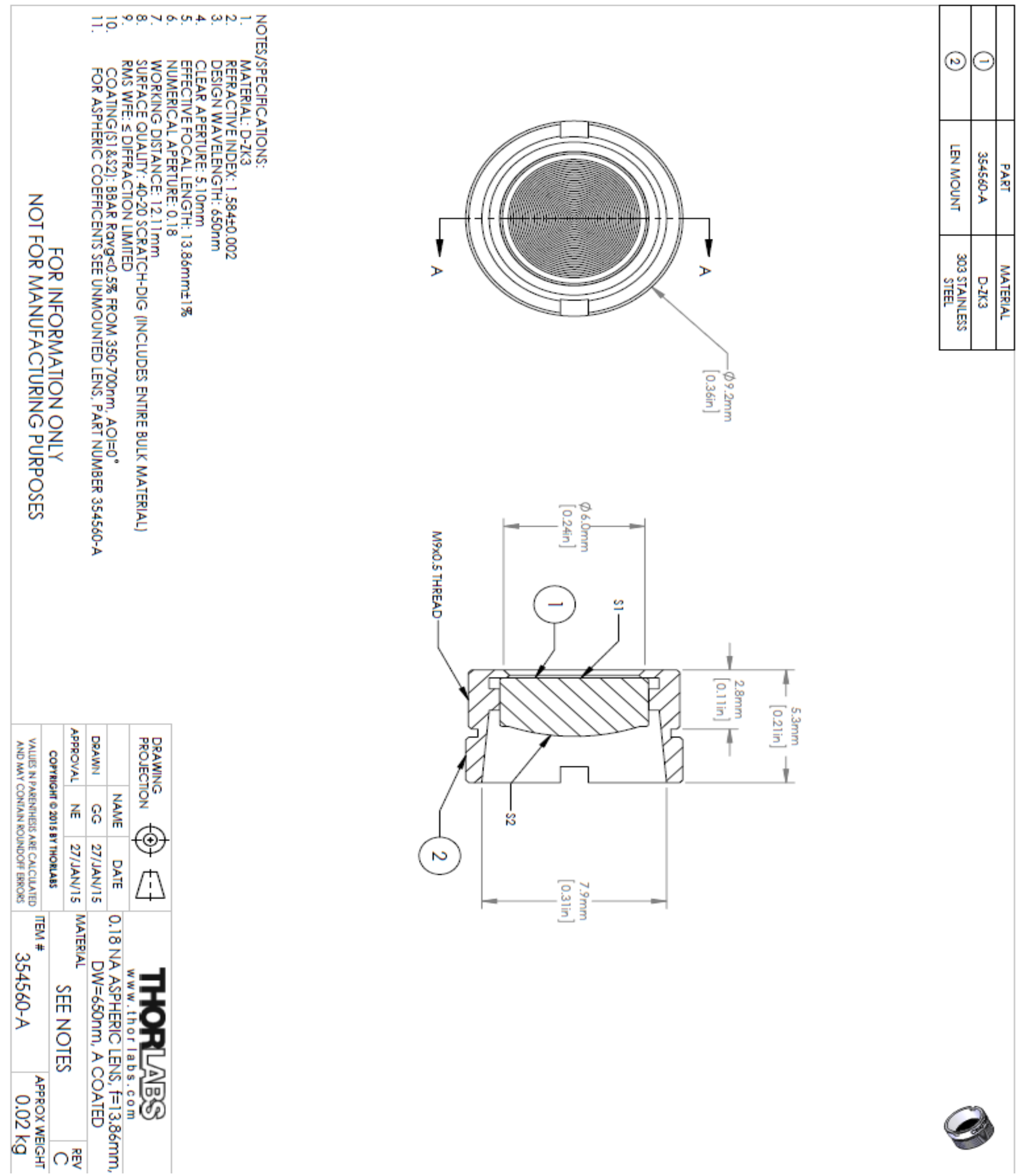


\section{F. Hamamatsu Photomultiplier Tube}

This Appendix contains specifications of the Hamamatsu photomultiplier tube. The H9305-02 is the PMT that is used in all channels on the optics truss. 


\section{Compact Side-on PMT \\ Photosensor Modules H9305 Series}

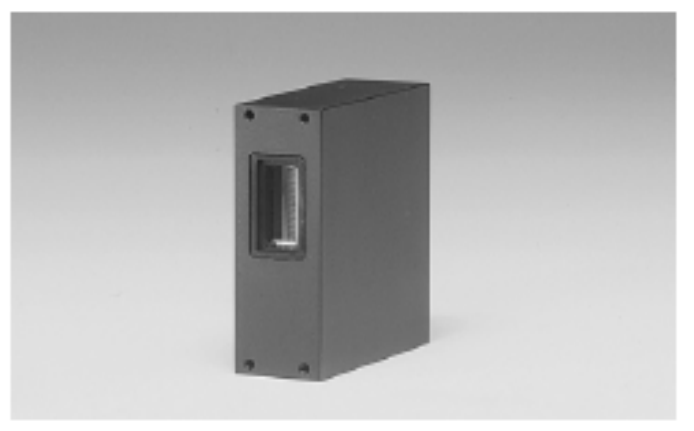

The H9305 series photosensor modules contain a high-voltage power supply circuit and a 13-mm (1/2") diameter side-on photomultiplier tube in a compact aluminum housing. The $13-\mathrm{mm}\left(1 / 2^{\prime \prime}\right)$ side-on photomultiplier tube has a reflection mode photocathode that delivers high quantum efficiency at wavelengths above $600 \mathrm{~nm}$, an adequate gain of up to $10^{7}$ and fast time response. High $\mathrm{S} / \mathrm{N}$ ratio can be obtained even when measuring extremely low level light at high speeds.

The H9305 series uses a Cockcroft-Walton circuit with low power consumption. Flexible cables are used for easy installation in equipment.

\section{Product Variations}

\begin{tabular}{l|c|l}
\hline \multicolumn{1}{c|}{ Type No. } & Spectral Response & \multicolumn{1}{c}{ Features } \\
\hline H9305-01 & $185 \mathrm{~nm}$ to $750 \mathrm{~nm}$ & High sensitivity in UV to visible range \\
\hline $\mathrm{H} 9305-02$ & $185 \mathrm{~nm}$ to $900 \mathrm{~nm}$ & For general applications in UV to near IR range \\
\hline $\mathrm{H} 9305-03$ & $185 \mathrm{~nm}$ to $900 \mathrm{~nm}$ & High sensitivity in UV to near IR range \\
\hline $\mathrm{H} 9305-04$ & $185 \mathrm{~nm}$ to $830 \mathrm{~nm}$ & Low dark current in UV to near IR range \\
\hline $\mathrm{H} 9305-05$ & $185 \mathrm{~nm}$ to $650 \mathrm{~nm}$ & For general applications in UV to visible range \\
\hline
\end{tabular}

This product can't be used at vacuum environment or reduced pressure environment. Please pay attention when the H9305 series is used for measuring the light below $190 \mathrm{~nm}$.

\section{Specifications}

$\left(\right.$ at $\left.+25^{\circ} \mathrm{C}\right)$

\begin{tabular}{|c|c|c|c|c|c|c|c|c|}
\hline \multicolumn{3}{|c|}{ Parameter } & \multicolumn{5}{|c|}{ H9305 Series } & \multirow{2}{*}{$\begin{array}{c}\text { Unit } \\
-\end{array}$} \\
\hline \multicolumn{3}{|c|}{ Suffix } & -01 & -02 & -03 & -04 & -05 & \\
\hline \multicolumn{3}{|c|}{ Input Voltage } & \multicolumn{5}{|c|}{+11.5 to +15.5} & $\mathrm{~V}$ \\
\hline \multicolumn{3}{|c|}{ Max. Input Voltage } & \multicolumn{5}{|c|}{+18} & $\mathrm{~V}$ \\
\hline \multicolumn{3}{|c|}{ Max. Input Current } & \multicolumn{5}{|c|}{7} & $\mathrm{~mA}$ \\
\hline \multicolumn{3}{|c|}{ Max. Output Signal Current } & \multicolumn{5}{|c|}{10} & $\mu \mathrm{A}$ \\
\hline \multicolumn{3}{|c|}{ Max. Control Voltage } & \multicolumn{5}{|c|}{ +1.2 (Input impedance: $1 \mathrm{M} \Omega$ ) } & $\mathrm{V}$ \\
\hline \multicolumn{3}{|c|}{ Recommended Control Voltage Adjustment Range } & \multicolumn{5}{|c|}{+0.25 to +1.0} & $\mathrm{~V}$ \\
\hline \multicolumn{3}{|c|}{ Effective Area } & \multicolumn{5}{|c|}{$3.7 \times 13.0$} & $\mathrm{~mm}$ \\
\hline \multicolumn{3}{|c|}{ Peak Sensitivity Wavelength } & 420 & 400 & 450 & 530 & 340 & $\mathrm{~nm}$ \\
\hline \multirow{5}{*}{$\frac{8}{\frac{8}{0}}$} & \multirow{2}{*}{ Luminous Sensitivity } & Min. & 80 & 200 & 350 & 140 & 20 & \multirow{2}{*}{$\mu \mathrm{A} / \mathrm{m}$} \\
\hline & & Typ. & 120 & 300 & 500 & 200 & 40 & \\
\hline & Blue Sensitivity Index (CS 5-58) & Typ. & 10 & - & - & - & 5 & 一 \\
\hline & Red/White Ratio & Typ. & - & 0.3 & 0.4 & 0.15 & - & - \\
\hline & Radiant Sensitivity ${ }^{* 1}$ & Typ. & 90 & 77 & 105 & 70 & 48 & mAWW \\
\hline \multirow{5}{*}{$\frac{9}{\frac{1}{8}}$} & \multirow{2}{*}{ Luminous Sensitivity •2 } & Min. & 100 & 400 & 1000 & 300 & 50 & \multirow{2}{*}{ Allm } \\
\hline & & Typ. & 700 & 1200 & 2000 & 700 & 300 & \\
\hline & Radiant Sensitivity ${ }^{* 1} * 2$ & Typ. & $5.2 \times 10^{5}$ & $3.1 \times 10^{5}$ & $4.2 \times 10^{5}$ & $2.5 \times 10^{5}$ & $3.6 \times 10^{5}$ & $\mathrm{~A} / \mathrm{W}$ \\
\hline & \multirow{2}{*}{ Dark Current $* 2 * 3$} & \begin{tabular}{|l|} 
Typ. \\
\end{tabular} & 1 & 1 & 2 & 0.1 & 0.5 & \multirow{2}{*}{$\mathrm{nA}$} \\
\hline & & Max. & 10 & 10 & 10 & 1 & 5 & \\
\hline \multicolumn{2}{|c|}{ Rise Time ${ }^{* 2}$} & Typ. & \multicolumn{5}{|c|}{1.4} & $\mathrm{~ns}$ \\
\hline \multicolumn{2}{|r|}{ Ripple Noise ${ }^{* 2 * 4}$ (peak to peak) } & Max. & \multicolumn{5}{|c|}{0.5} & $\mathrm{mV}$ \\
\hline Set & tling Time ${ }^{* 5}$ & Max. & & & 10 & & & $s$ \\
\hline Op & erating Ambient Temperature & $e^{+6}$ & & & +5 to +50 & & & ${ }^{\circ} \mathrm{C}$ \\
\hline Sto & rage Temperature ${ }^{* 6}$ & & & & -20 to +50 & & & ${ }^{\circ} \mathrm{C}$ \\
\hline We & ight & & & & 110 & & & $g$ \\
\hline
\end{tabular}

"1: Measured at the peak sensitivity wavelength

2 : Control voltage $=+1.0 \mathrm{~V}$

"3: After 30 minutes storage in darkness

"4: Cable RG-174/U, Cable length $450 \mathrm{~mm}$, Load resistance $=1 \mathrm{M} \Omega$, Load capacitance $=22 \mathrm{pF}$

"5: The time required for the output to reach a stable level following a change in the control voltage from $+1.0 \mathrm{~V}$ to $+0.5 \mathrm{~V}$.

"6: No condensation 


\section{Current Output Type Photosensor Modules}

\section{Characteristics (Cathode radiant sensitivity, PMT gain)}
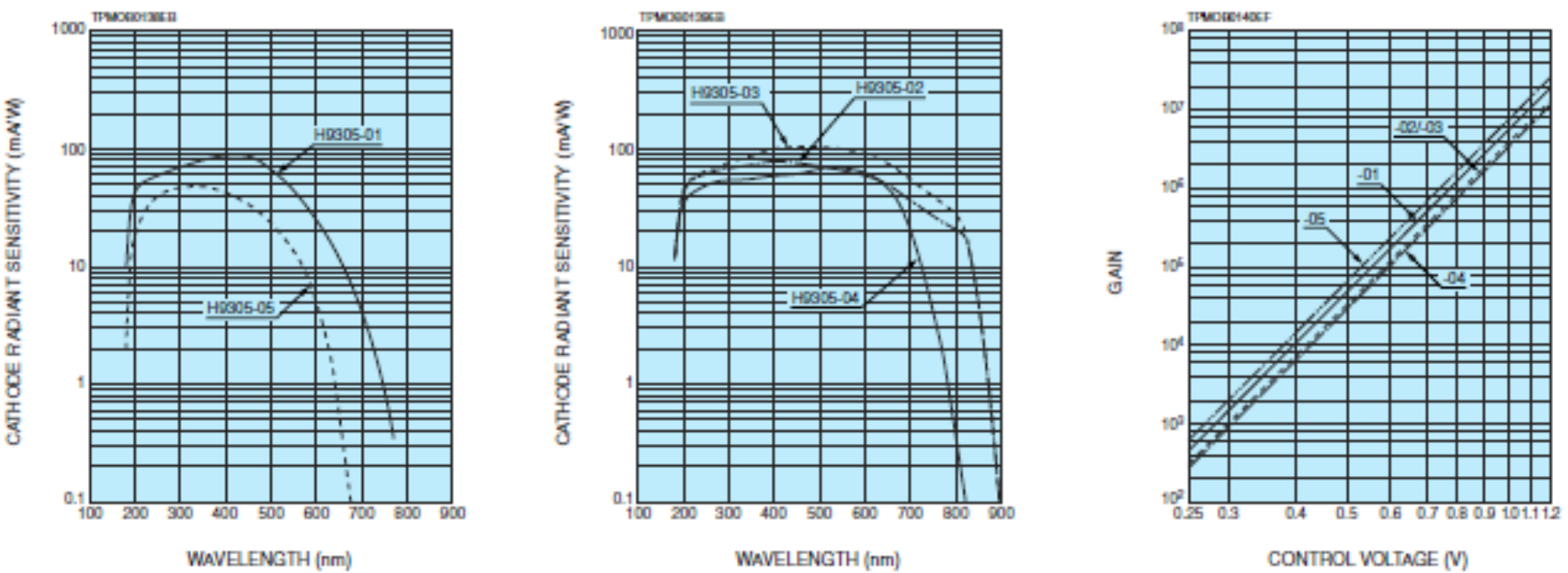

\section{Sensitivity Adjustment Method}

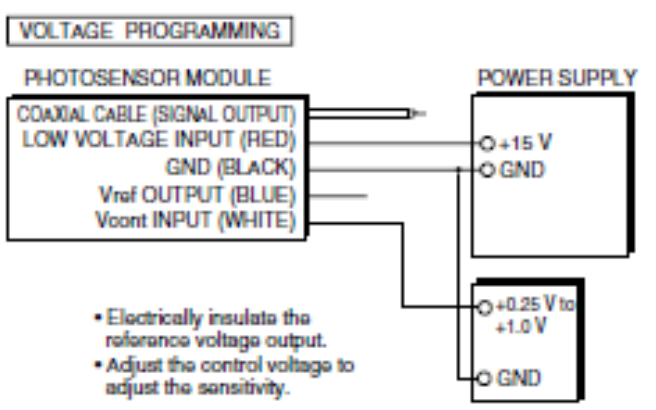

RESISTTANCE PROGRAMMING

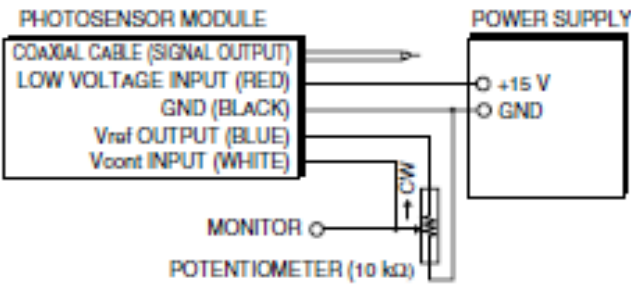

When using a potentiometor, adjust sensitivity whilo monitoring the control voltage so it does not axceod $+1.2 \mathrm{~V}$.

\section{Dimensional Outlines (Unit: mm)}
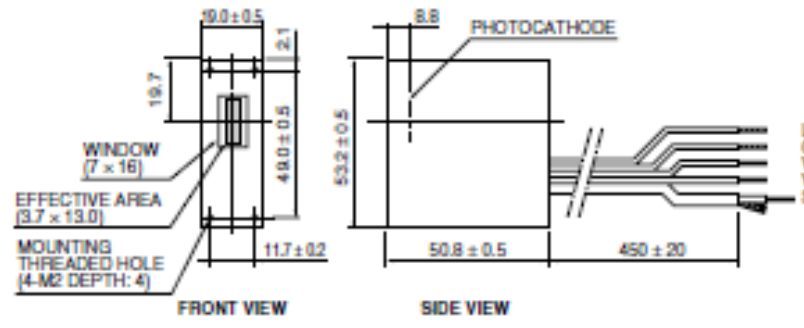

LOW VOLTAGE NFUT $(+15 \mathrm{~V})$ - AWCOE

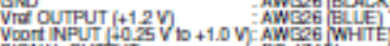
SIGWL OUTAUT

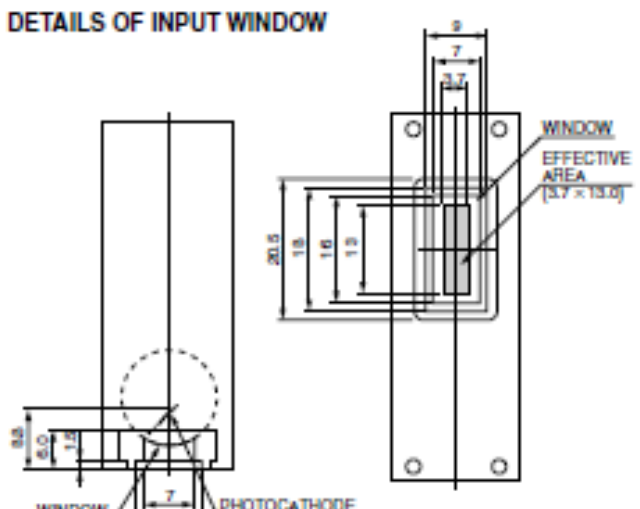




\section{G. Spherotech Rainbow Calibration Beads}

This Appendix contains specification on the Spherotech Beads. These beads are used to calibrate cytometers. The beads contain 8 populations of spheres all with the same diameter. The 8 populations range from no fluorescent signal to high fluorescent signal. Using these known specifications allows a user to be sure that all signal is being seen in a cytometer. 
Spherotech, Inc.

27845 Ima Lee Gincle, Unit 101, Loke Forest, IL 60045

\section{SPHERO ${ }^{\text {TM }}$ Calibration Particles}

SPHERO' Calibration Particles are designed for routine calibration of flow cytometers. They are used extensively by many laboratories for $\mathrm{QC}$ and long term performance turacking of the flow cytonneter. In addition, they are also used for routine alignment and calibration in fluorescence and confocal fluorescence microscopy.

\section{SPHERO ${ }^{T M}$ Rainbow Calibration Particles}

- Contains multiple fluorophores incorporated in the same particle to be used in multiple channels of the flow cytometer.

* Available with different fluorescent intensities on the same size particles.

- Stable for several years when stored properly

* Withstand freeze-thaw cycles; diluted particles can be stored frozen for later use.

- Can be sanitized by treating with $70 \%$ ethanol or other antibiotic agents.

The Rainbow Calibration Particles (RCPs) contain a mixture of several similar size particles with different fluorescence intensities. Every particle contains a moxture of fluorophores that al lows excitation at any wavelength from 365 to $650 \mathrm{~nm}$. As a result, most channels in the flow cytometer can be calibrated using the same set of particles.

These particles are also used to determine the relative woltage range for each flow cytometry detector. This will determine the dynamic range of specific PI-1T detectors:

*Perfetto,S.P., DAmbrozak et al. (2006). "Quality assurance for polychromatic flow cytometry" Plat. Protocols I(3): $1522-1530$.

\begin{tabular}{|c|c|c|c|}
\hline Fartlele Trew and St fase & $5=w_{1}, \mathrm{~m}$ & Galas $\mathrm{Ha}$ & Un: \\
\hline 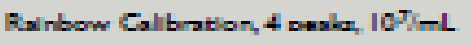 & $1.8-22$ & $x=725$ & $5 \mathrm{ml}$ \\
\hline Rarkon Galbatan, 6 aska, $107 / L$ & 20.3 .4 & nctos & $5 \mathrm{ml}$ \\
\hline 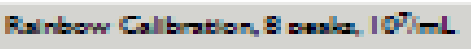 & 30.4 & AcF.30s & $5 \mathrm{~mL}$ \\
\hline 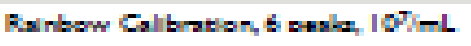 & 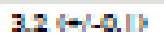 & AcF.32.5 & $5 \mathrm{ml}-$ \\
\hline 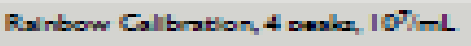 & $35-40$ & $A=75.5$ & $5 \mathrm{ml}$ \\
\hline 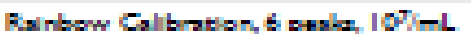 & 10.64 & $A D=-0.5$ & $5 \mathrm{ml}$ \\
\hline
\end{tabular}

Figure I The contour plot below displays the bead distributions of the Rainbow Calibration Particles in FITC vs PE (Catalog No. RCP-30-5A).

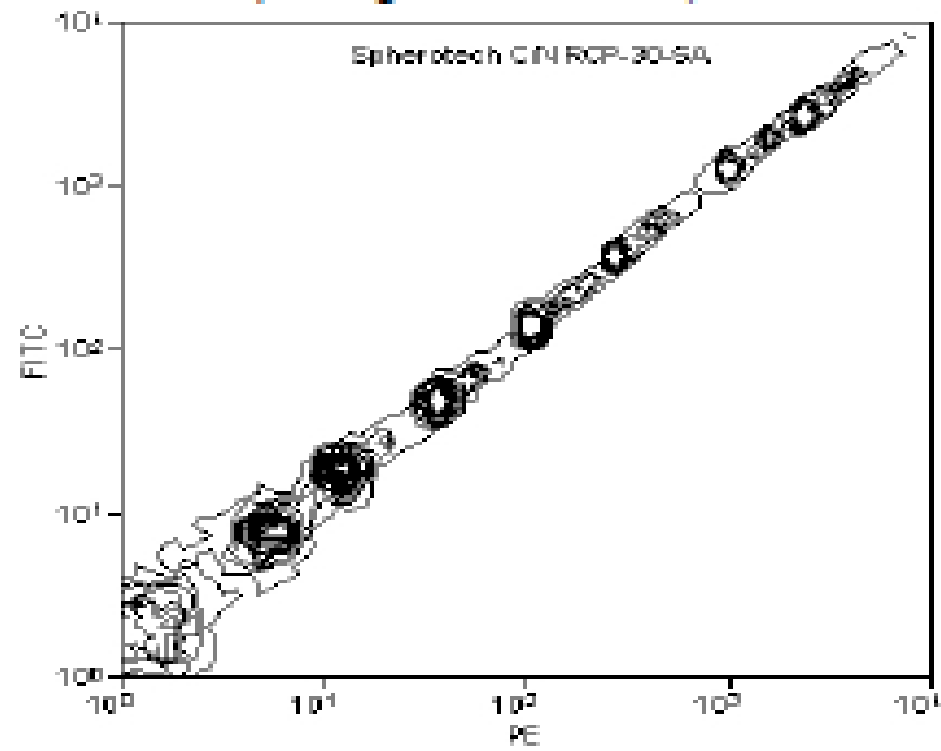

The RCPs provide a reliable and reproducible particle mixture for calibrating flow cytometers. They are very stable since the fluorochromes are entrapped within the particles instead of being located on the surface. In addition, Spherotech uses fluorophores that are non-spectral matching to the commonly used fluorophores such as FITC, FE or PE-Cy5. As a result, the RCPs are stable in terms of fluorescence.

The RCPs are convenient and affordable to use for long term performance tracking or routine calibration. They are packaged in a dropper bottle to facilitate the dispensing and storage. The diluted particles can be stored in the freezer for later use if desired to reduce costs. Dilution of a few drops of the partides from the dropper bottle to I $\mathrm{mL}$ of a diluent will provide adequate particle concentration for flow cytometer calibration. The diluted Rainbow Calibration Particles remain stable following repeated freezing and thawing- 
Spherotech, Inc.

27845 Irma Lee Circle, Unit IOI, Lake Forest, IL 60045

Figure 2 Histograms showing individual peaks representing various fluorescence intensities in Rainbow Calibration Particles (Catalog No. RCP-30-5A. Lot No. AAOI) are shown below.
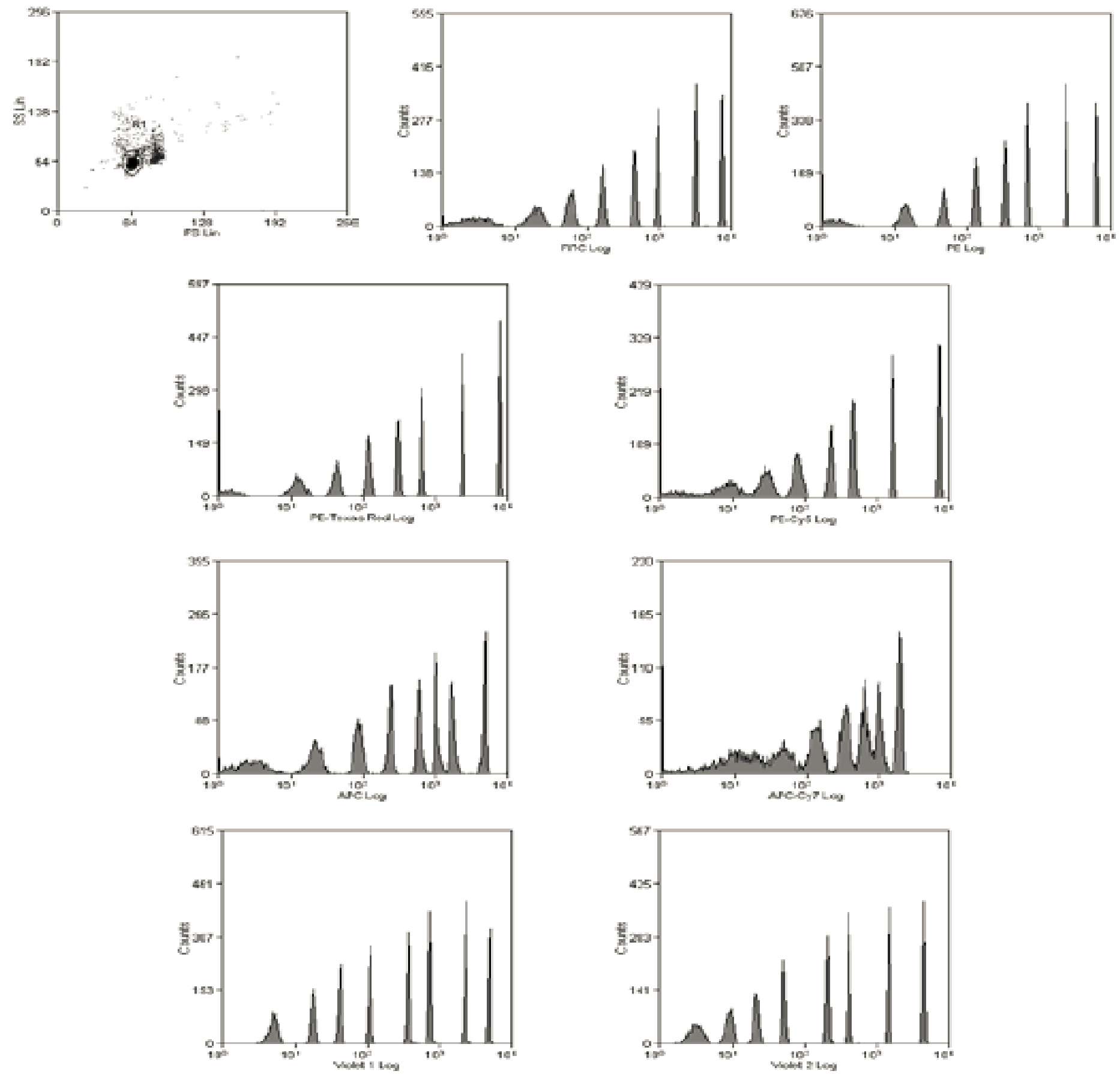
Spherotech, Inc.

27845 Irma Lee Circle, Unit I0I, Lake Forest, IL 60045

Figure 3 Histograms of the Rainbow Calibration Particles (Cat. No. RCP-30-5A, Lot No. AAOI) on a BD FacsCanto ${ }^{\text {TM II }}$
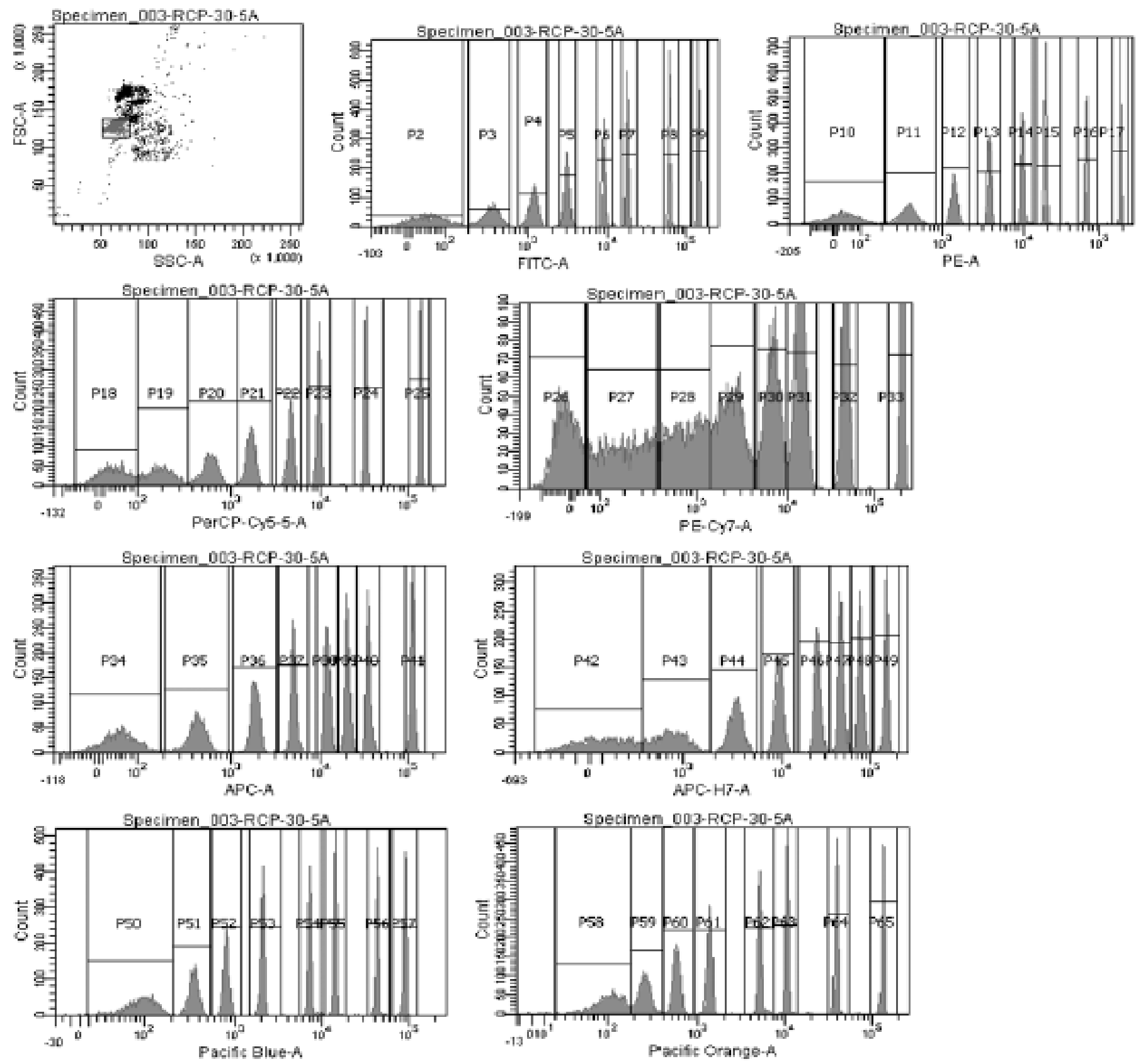

* Data provided by Laura Marszalek, Northwestern Memorial Hospital. 
Spherotech, Inc.

27845 Irma Lee Circle, Unit IOI, Lake Forest, IL 60045

Figure 4 Histograms of the Rainbow Calibration Particles (Cat. No. RCP-30-5A, Lot No. AAOI) on a BD LSR ${ }^{\mathrm{TM}}$ II
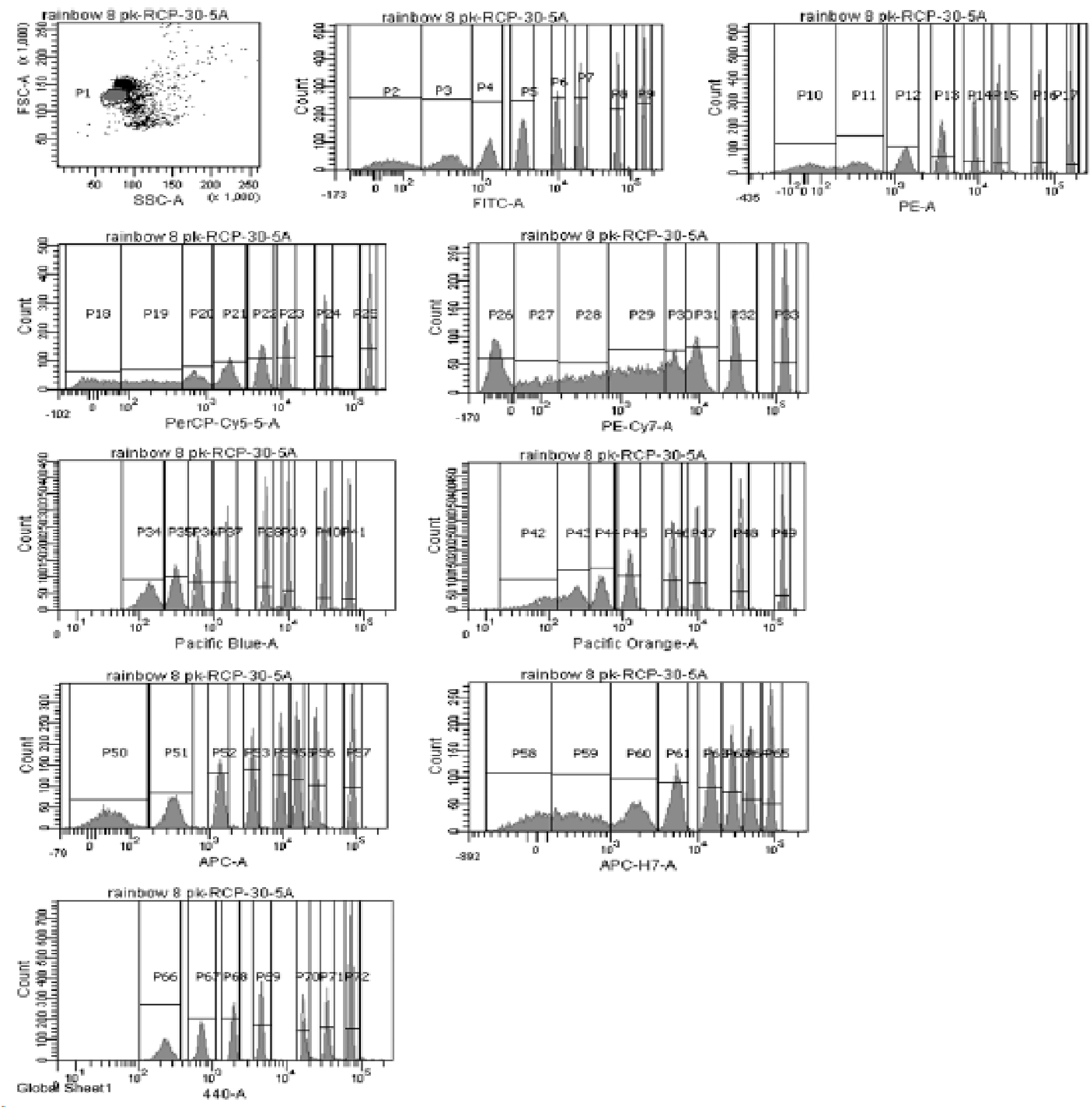

* Data provided by Laura Marszalek. Northwestern Memorial Hospital. 
Spherotech, Inc.

27845 Irma Lee Circle, Unit IOI, Lake Forest, IL 60045

The relative number of fluorophores per particles has been determined for every peak of RCP-30-5 (Lot\# $A A 01$ ) in FLI (FITC, MEFL). FL2 (RPE, MEPE), FL3 (RPECy5, MEPCY) and FL4 (APC, MEAP) channels of flow cytometer to plot the calibration graph as shown below. The calibration graph is used to check the linearity of the PMT in each channel. In addition, the relative number of fluorophores can be cross calibrated with cells or particles stained with known number of spectral matching fluorophores such as FITC, PE, RPE-Cy5 to estimate the number of fluorophores on stained cells. The RCP-30$5 \mathrm{~A}$, which is identical to RCP-30-5 with the exception of two additional peaks between the blank and the dimmest peak of RCP-30-5 to give a total of 8 peaks is shown on Page I. The RCP-30-5A is very useful in checking the sensitivity and resolution of the flow cytometer.

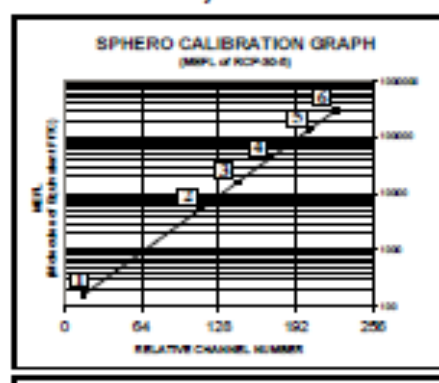

$$
\begin{aligned}
& \text { PEAK } \\
& 1 \\
& 2 \\
& 3 \\
& 4 \\
& 5 \\
& 6 \\
&
\end{aligned}
$$
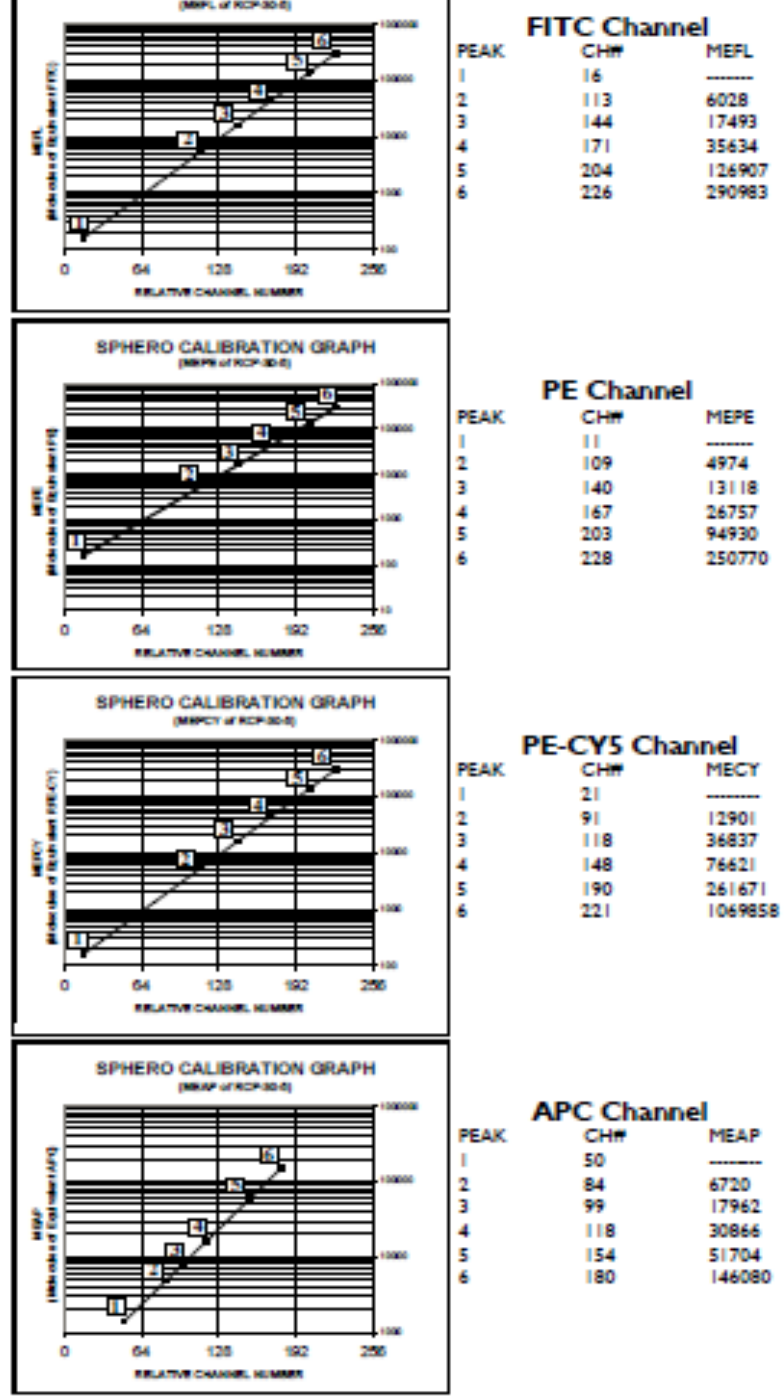

PMT QC Templates, as shown below, are downloadable. The template will allow the user to check and report the linearity of PMT in all channels easily by using RCP-30-5, RCP-30-5A, RCP-60-5, URCP38-2K, URCP-50-2K, RQC-4K or ACP-30-5K.

\section{PMT LINEARITY QC RECORD}

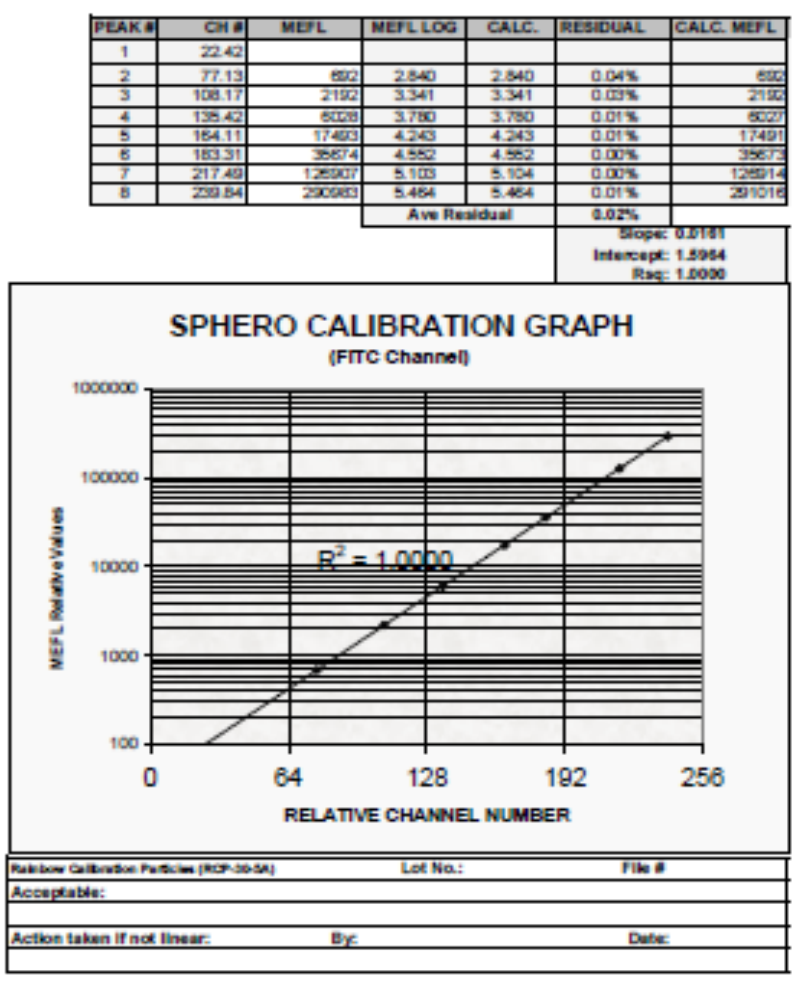

Figure 5 The Rainbow Calibration Particles are available in sizes of 2 to $6 \mu \mathrm{m}$ to suit various applications. Additional histograms of Rainbow Calibration Particles are shown on Pages 6-8.
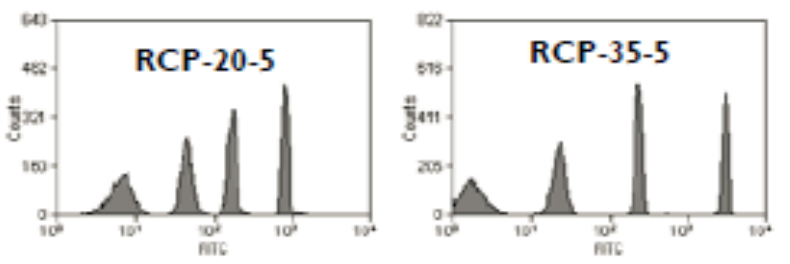
Spherotech, Inc.

27845 Irma Lee Circle, Unit I0I, Lake Forest, IL 60045

Figure 6 Histograms of the Rainbow Calibration Particles (Cat. No. RCP-60-5, Lot No. AA0I)
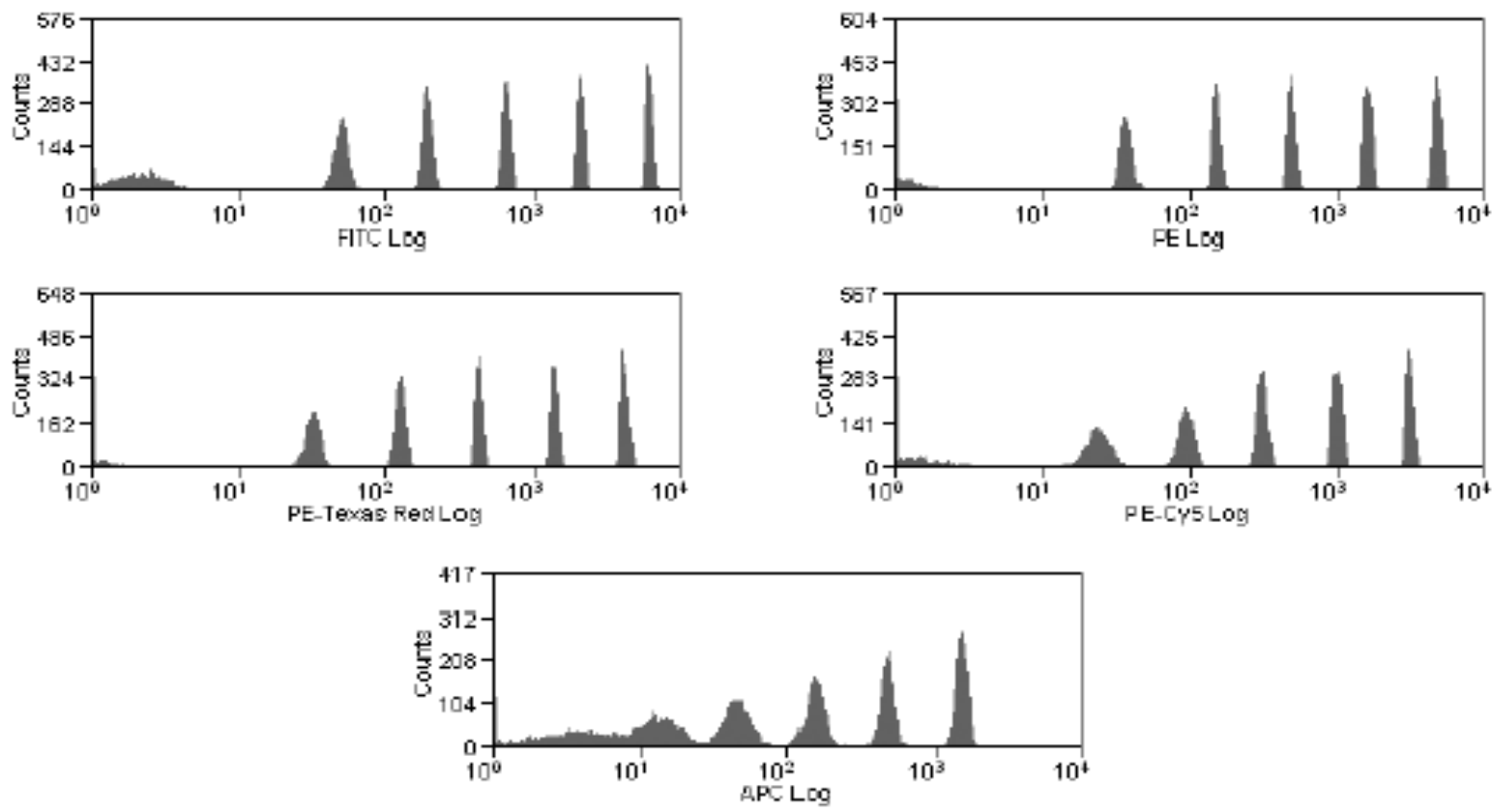

Figure 7 Histograms of the Rainbow Calibration Particles (Cat. No. RCP-32-5, Lot No. X0I-R)
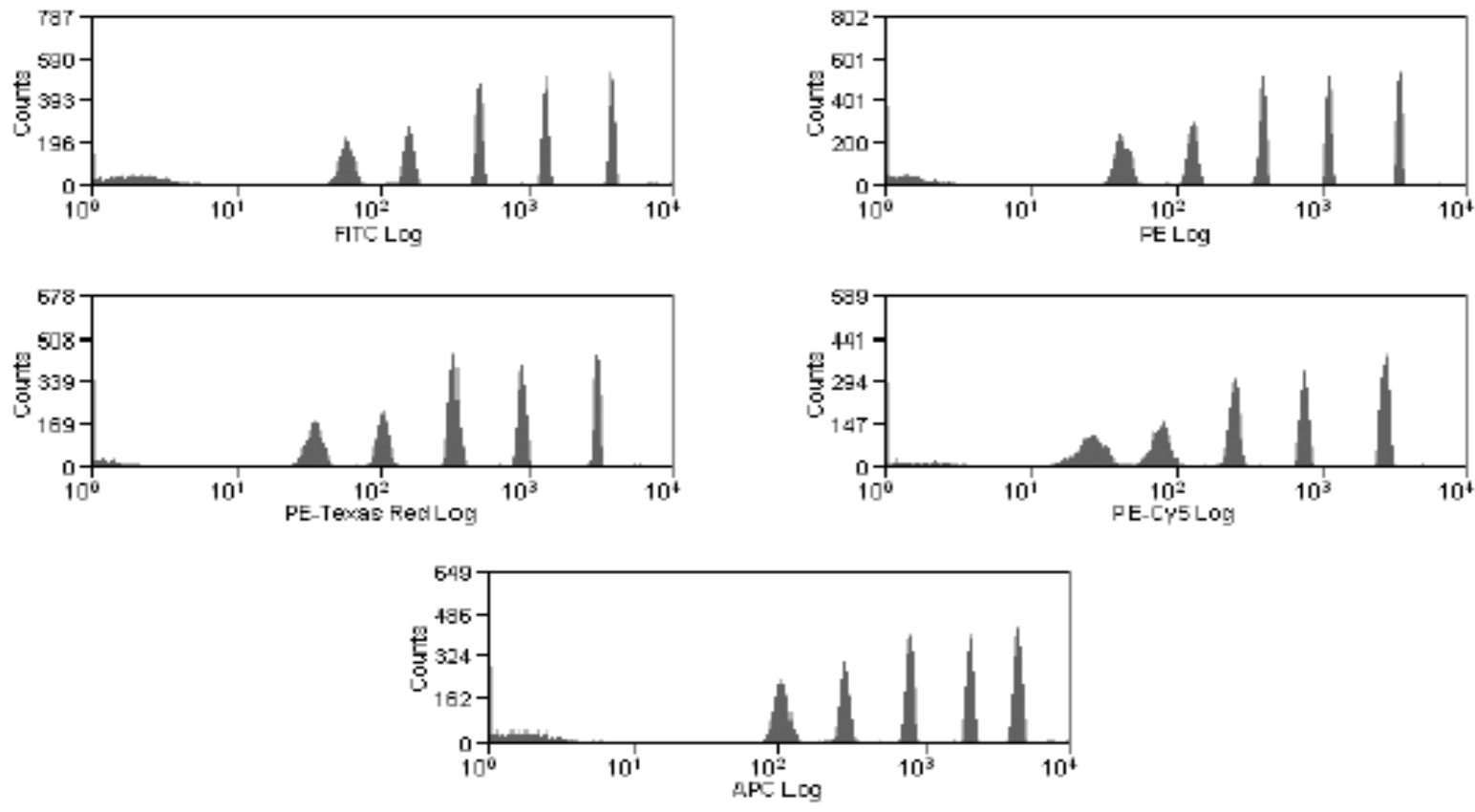
Spherotech, Inc.

27845 Irma Lee Circle, Unit I0I, Lake Forest, IL 60045

Figure 8 Histograms of the Rainbow Calibration Particles (Cat. No. RCP-30-5, Lot No. AA0I) on a BD FacsCanto ${ }^{\text {IM }}$ II
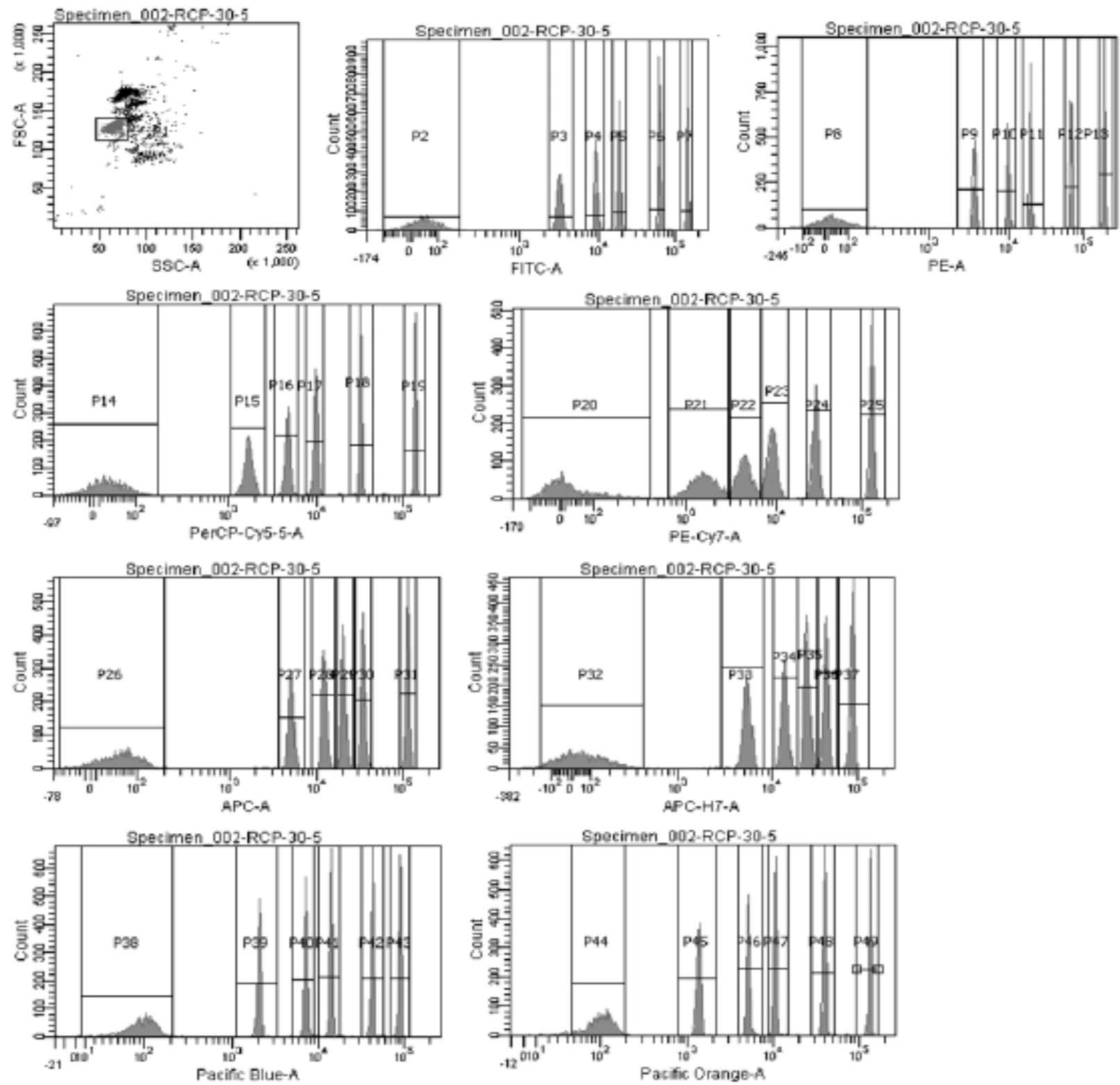

* Data provided by Laura Marszalek, Northwestern Memorial Hospital. 
Spherotech, Inc.

27845 Irma Lee Circle, Unit IOI, Lake Forest, IL 60045

Figure 9 Histograms of the Rainbow Calibration Particles (Cat. No. RCP-30-5, Lot No. AA0I) on a BD LSR ${ }^{\text {TM II }}$
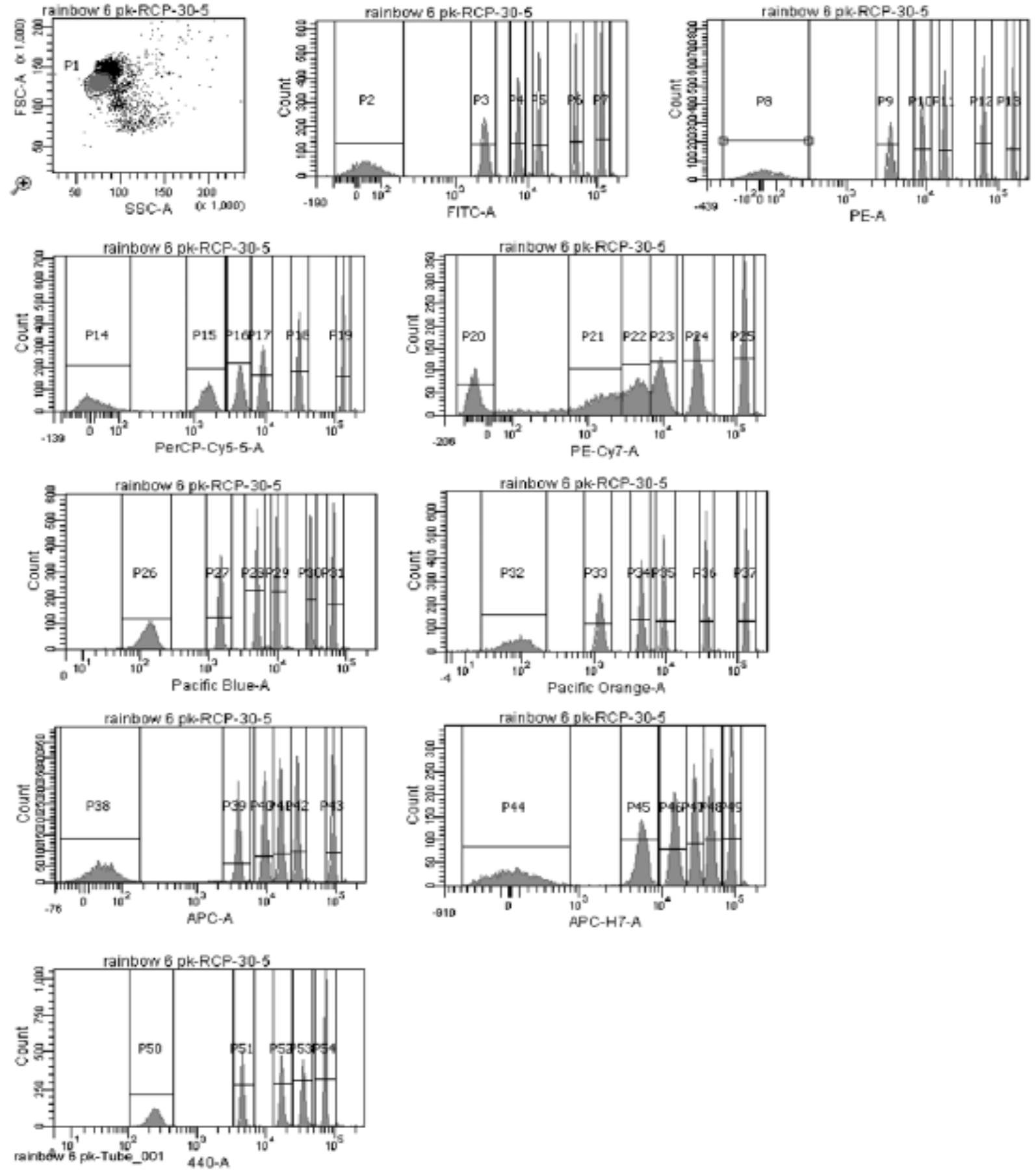

* Data provided by Laura Marszalek, Northwestern Memorial Hospital. 


\section{H. Laser Shape Testing Images}

This Appendix contains screen captures relating to the tests done in laser shape testing. These images contain the forward scatter, FL1, and FL2 views of the machine. There are three sets of data which are Baseline, testing with 100 focal length plano convex lens, and testing with a 200 focal length plano convex lens. 


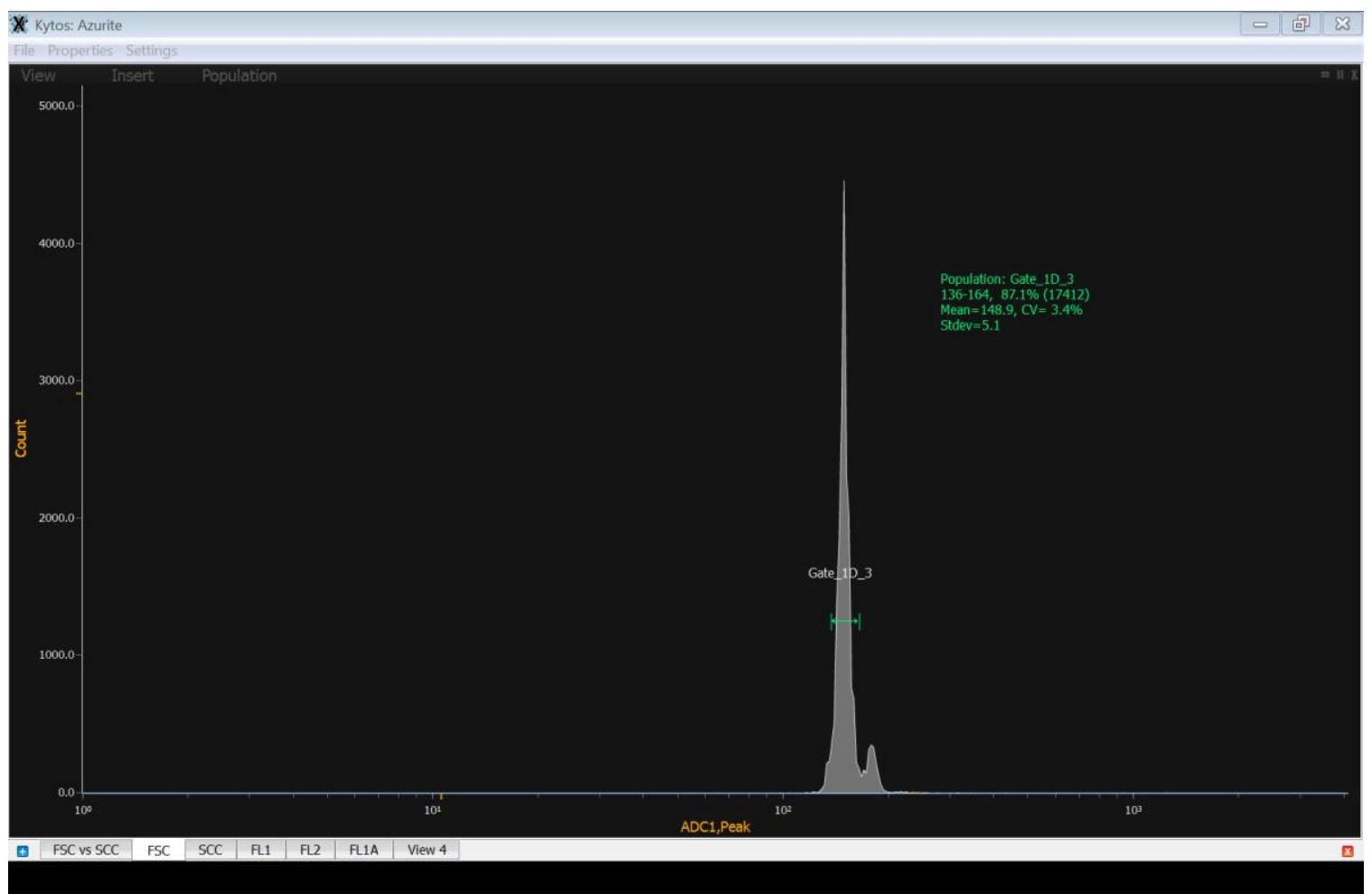

FSC Baseline

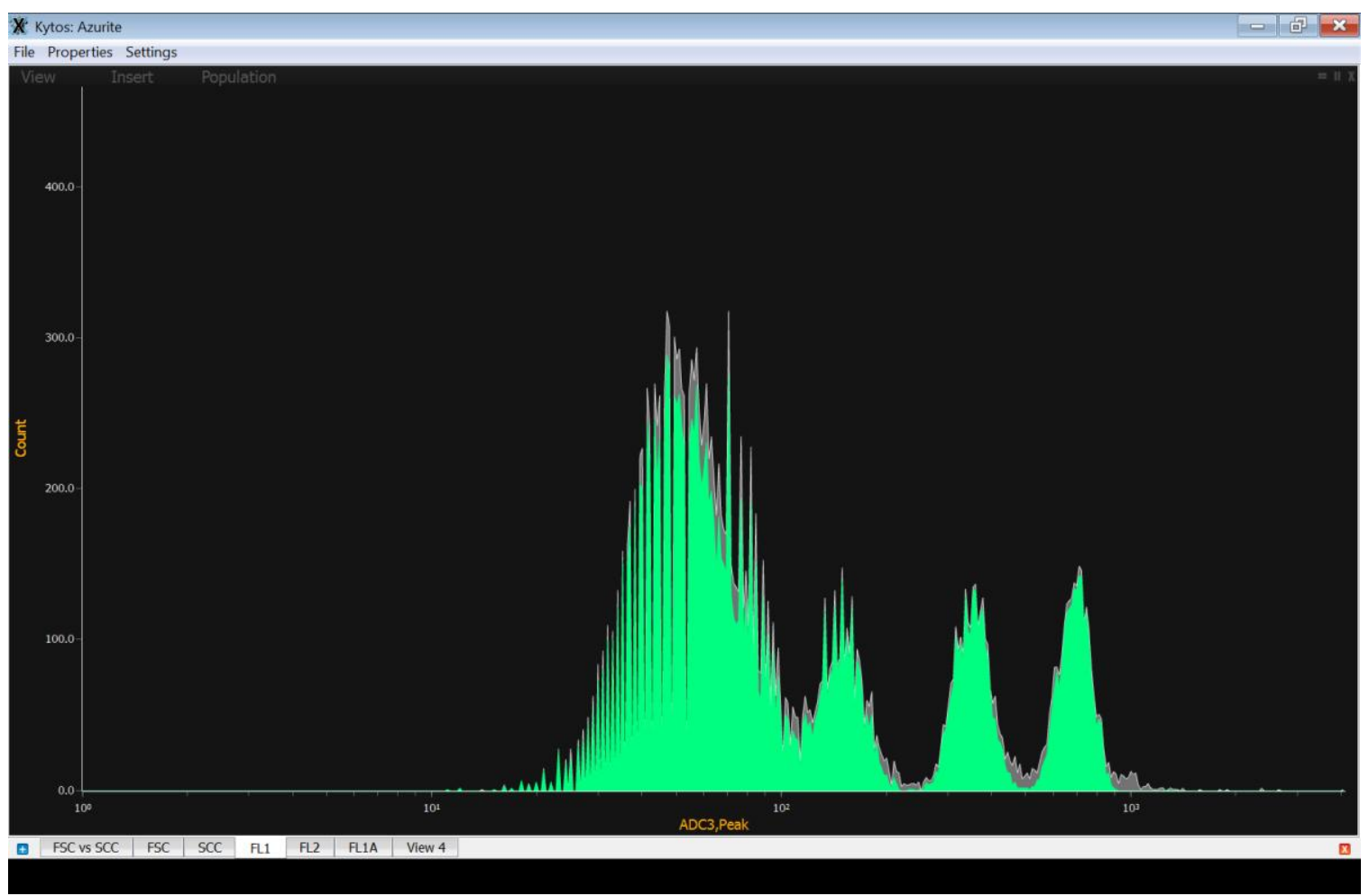

FL1 Baseline 


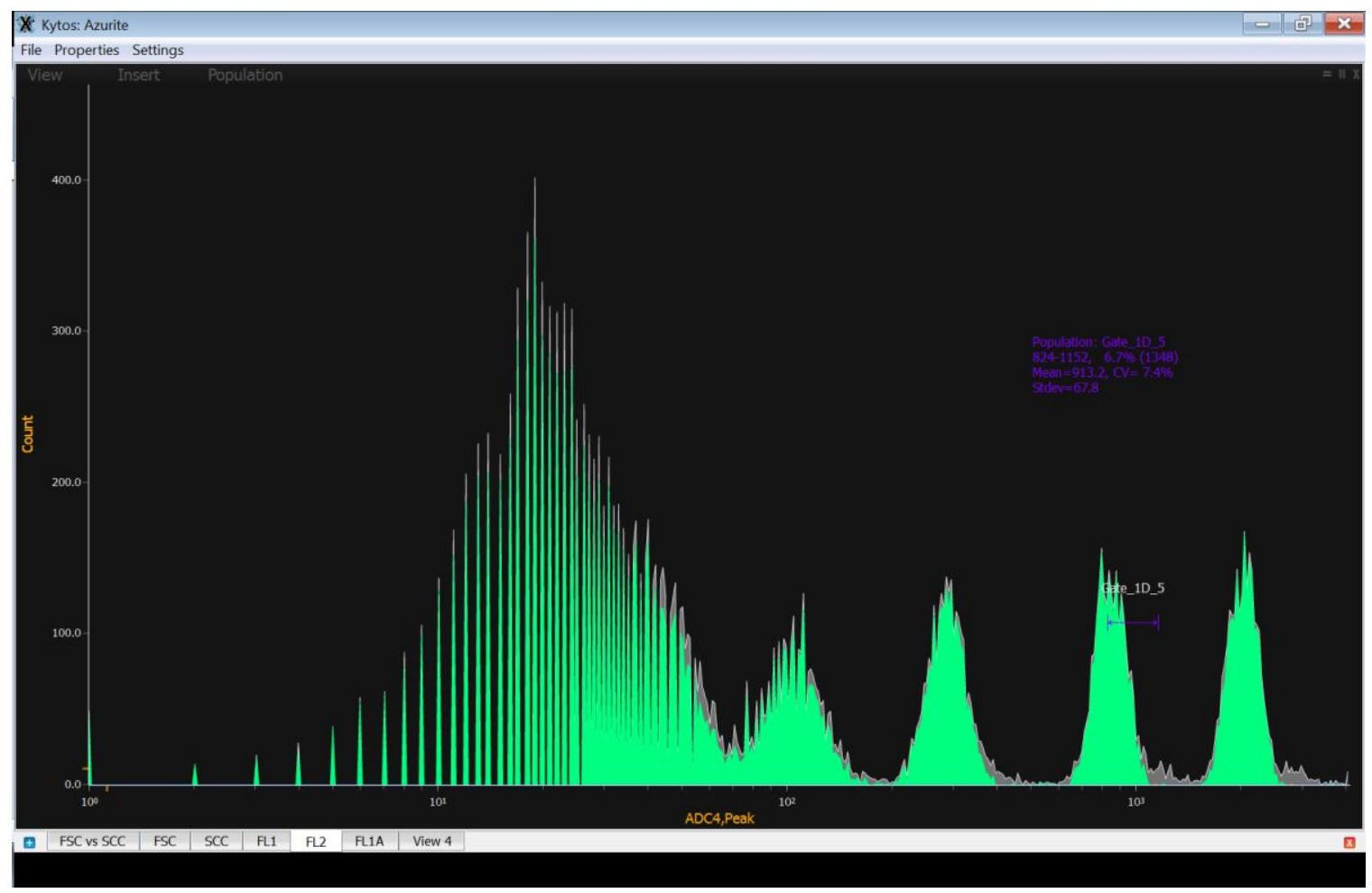

FL2 Baseline

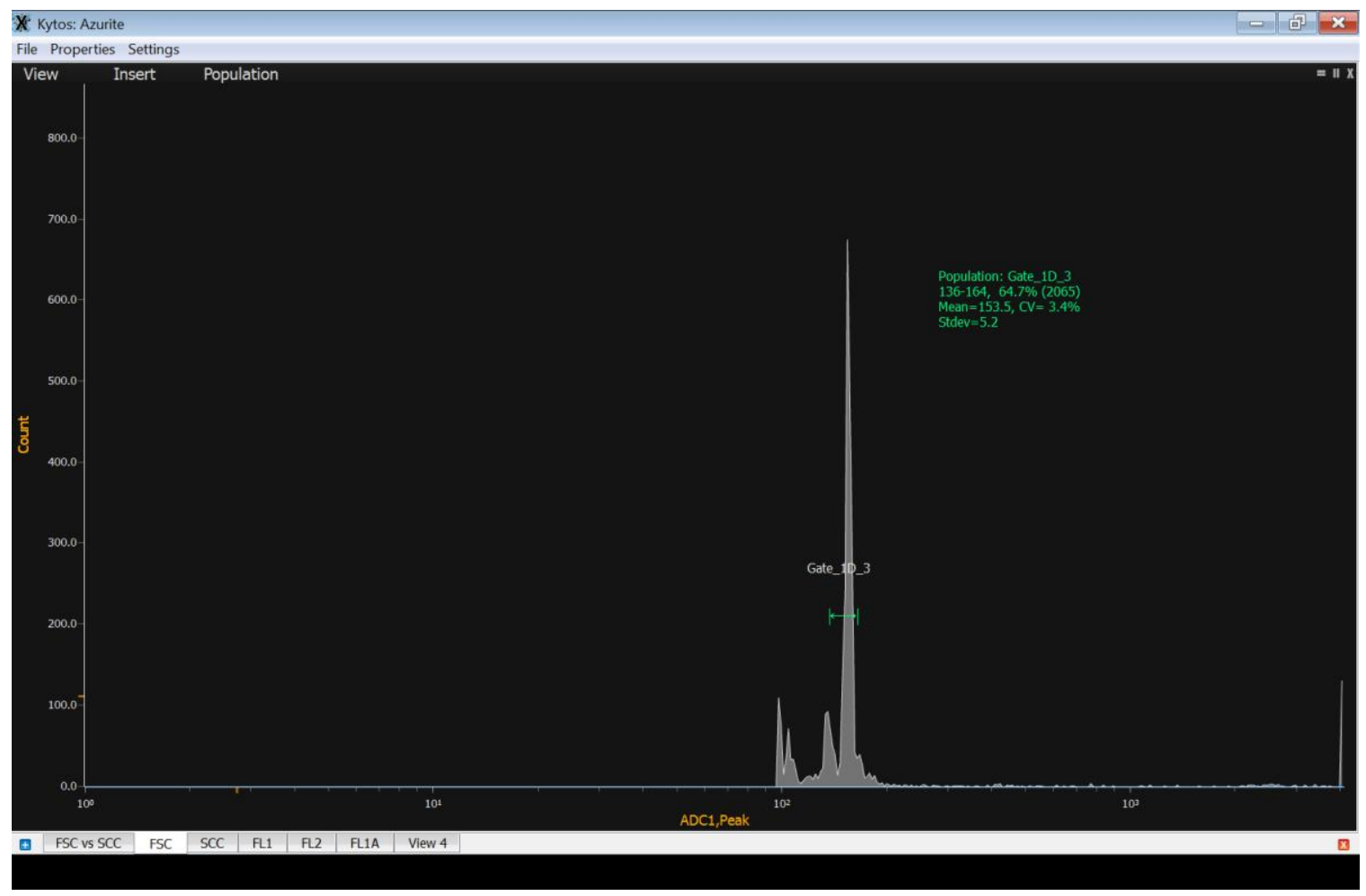

FSC $100 \mathrm{~mm}$ focal lens 


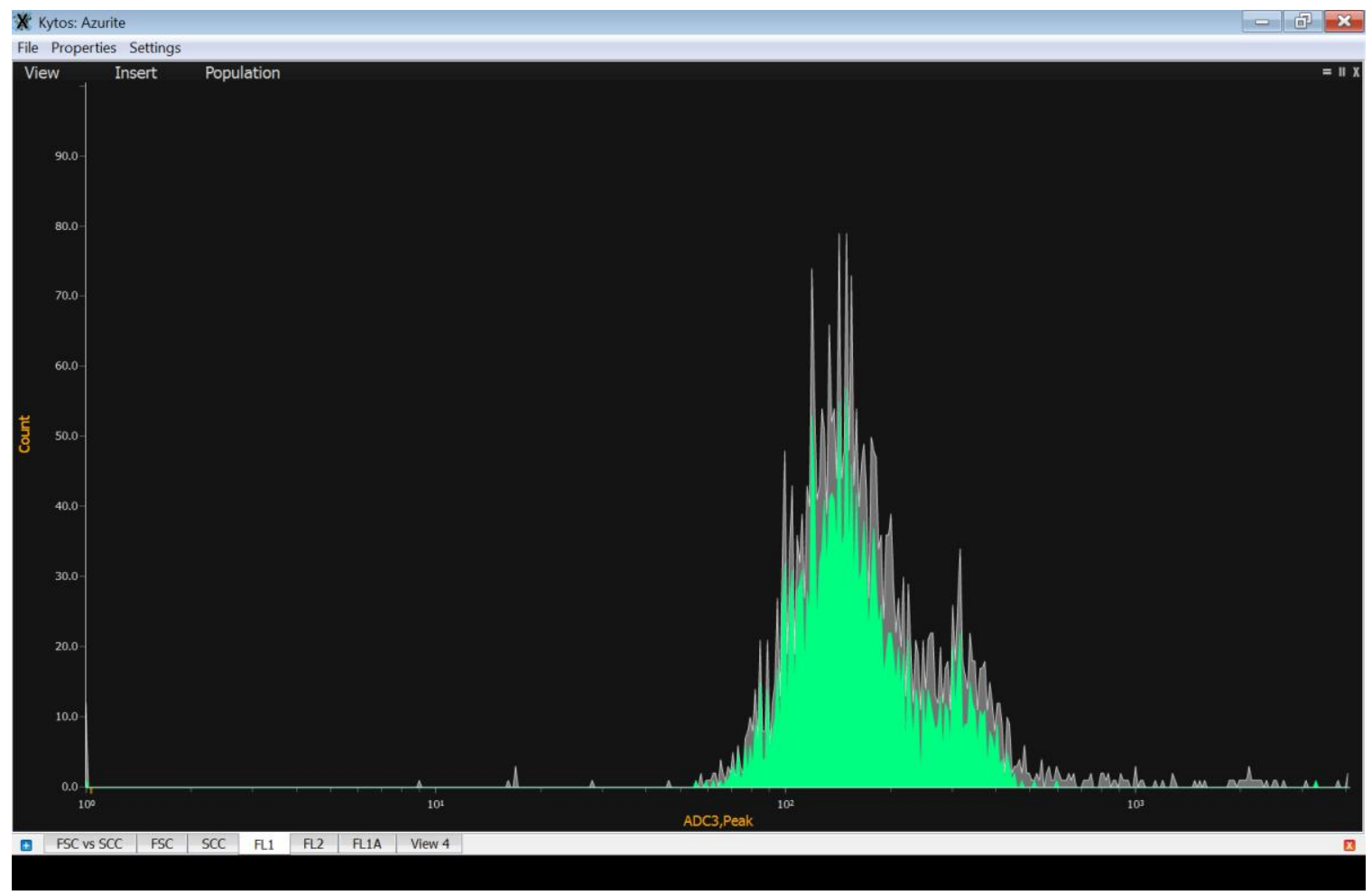

FL1 100mm focal lens

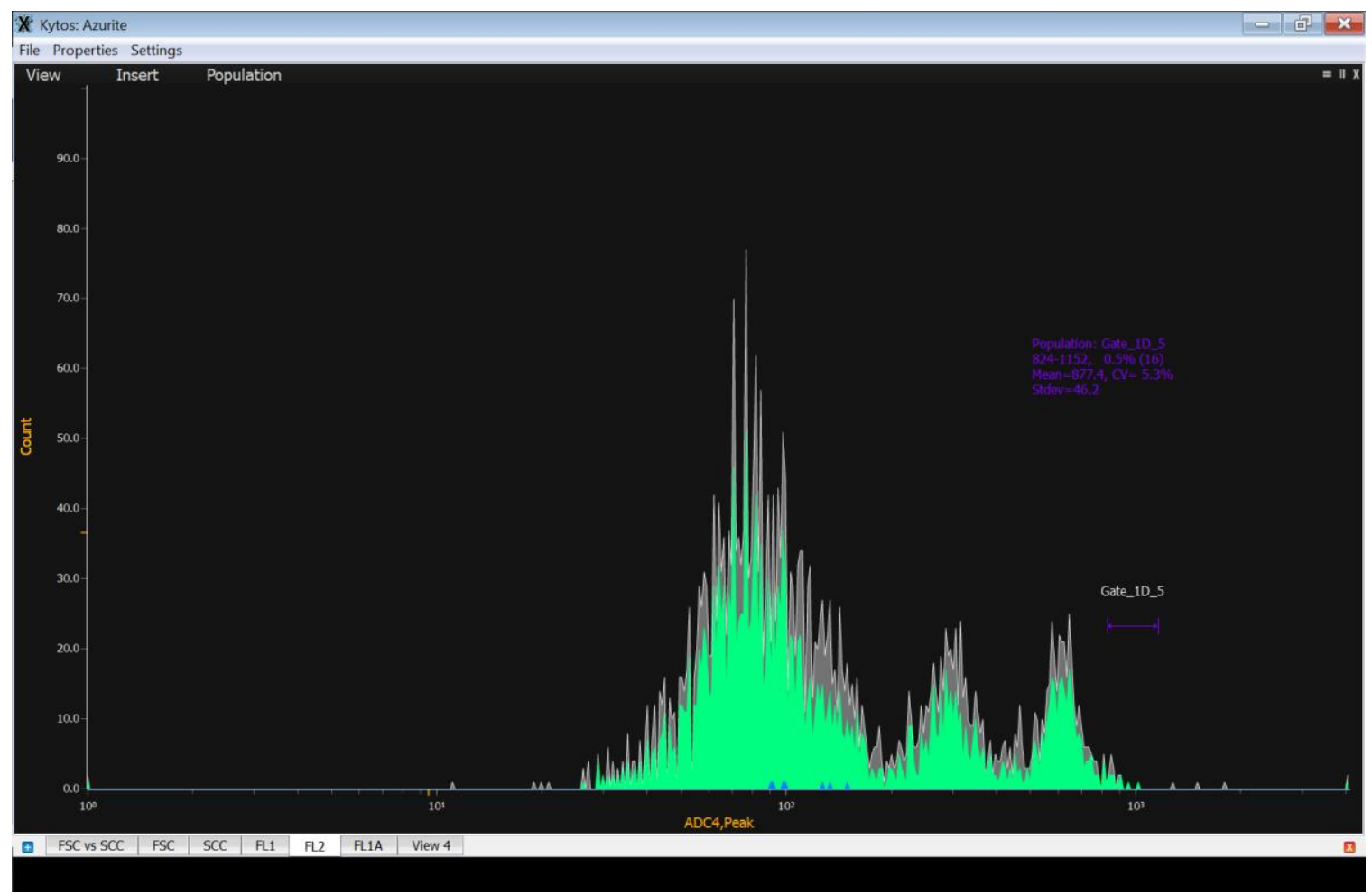

FL2 100mm focal lens 


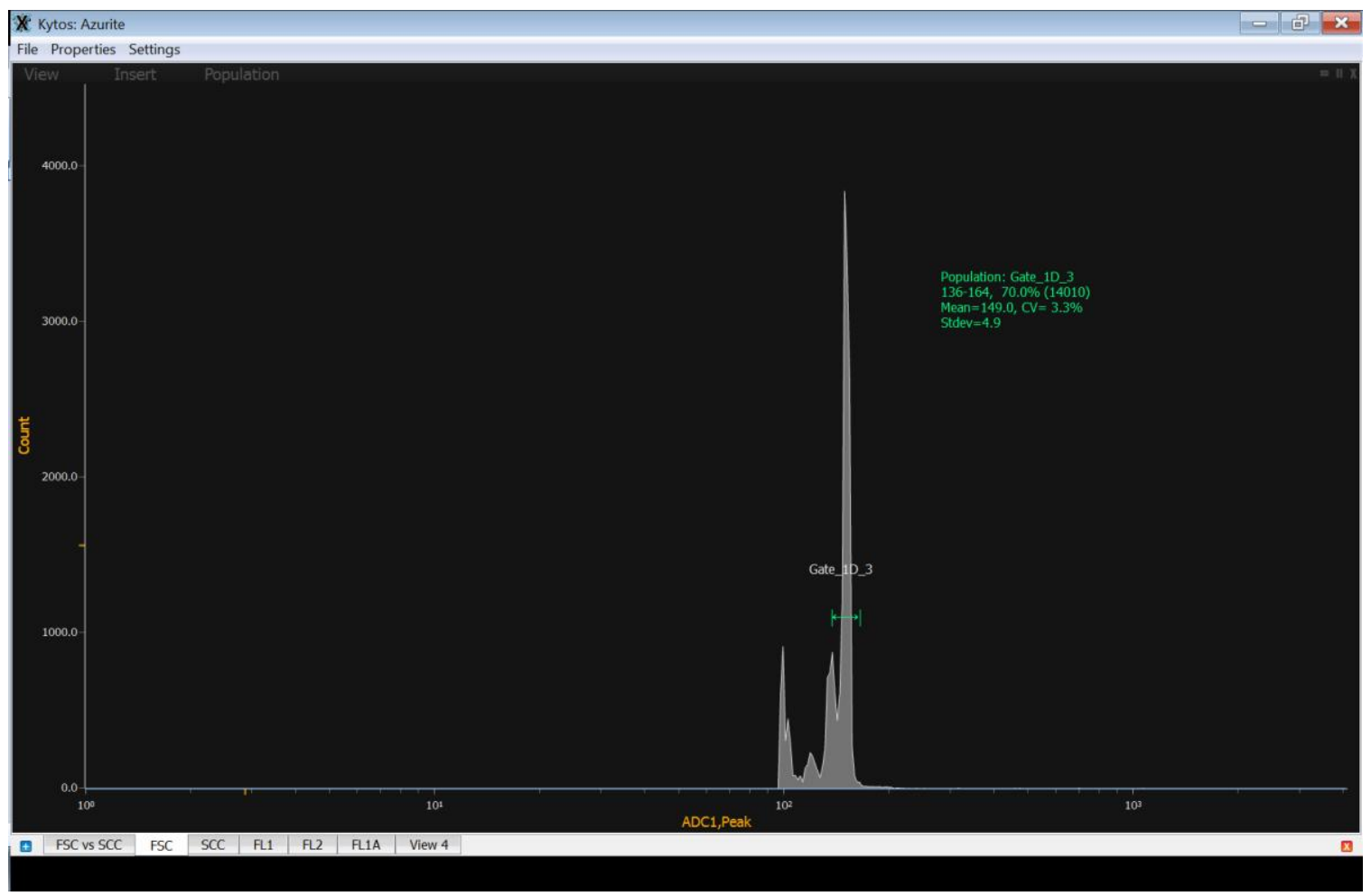

FSC $200 \mathrm{~mm}$ focal lens

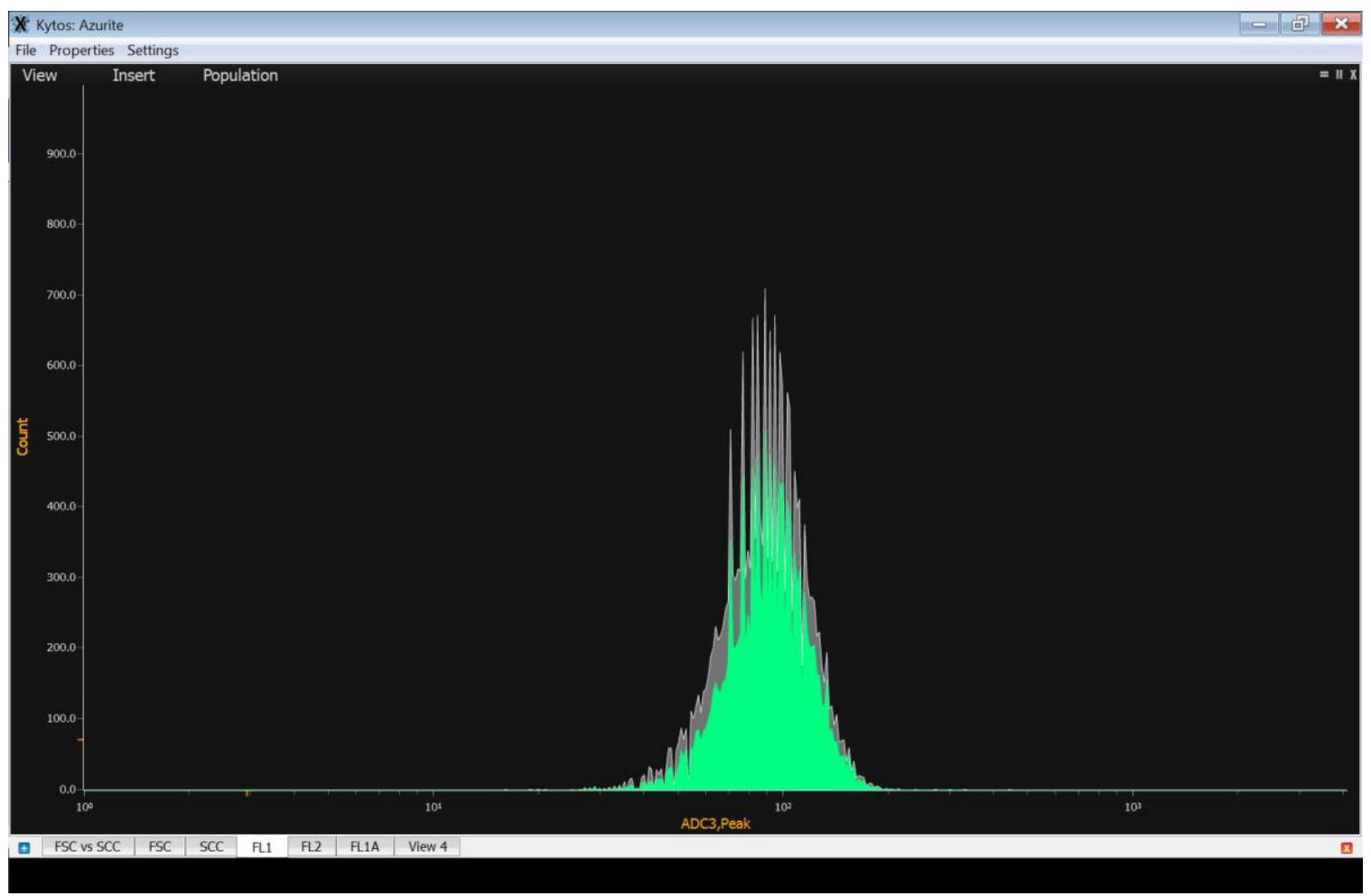

FL1 200mm focal lens 


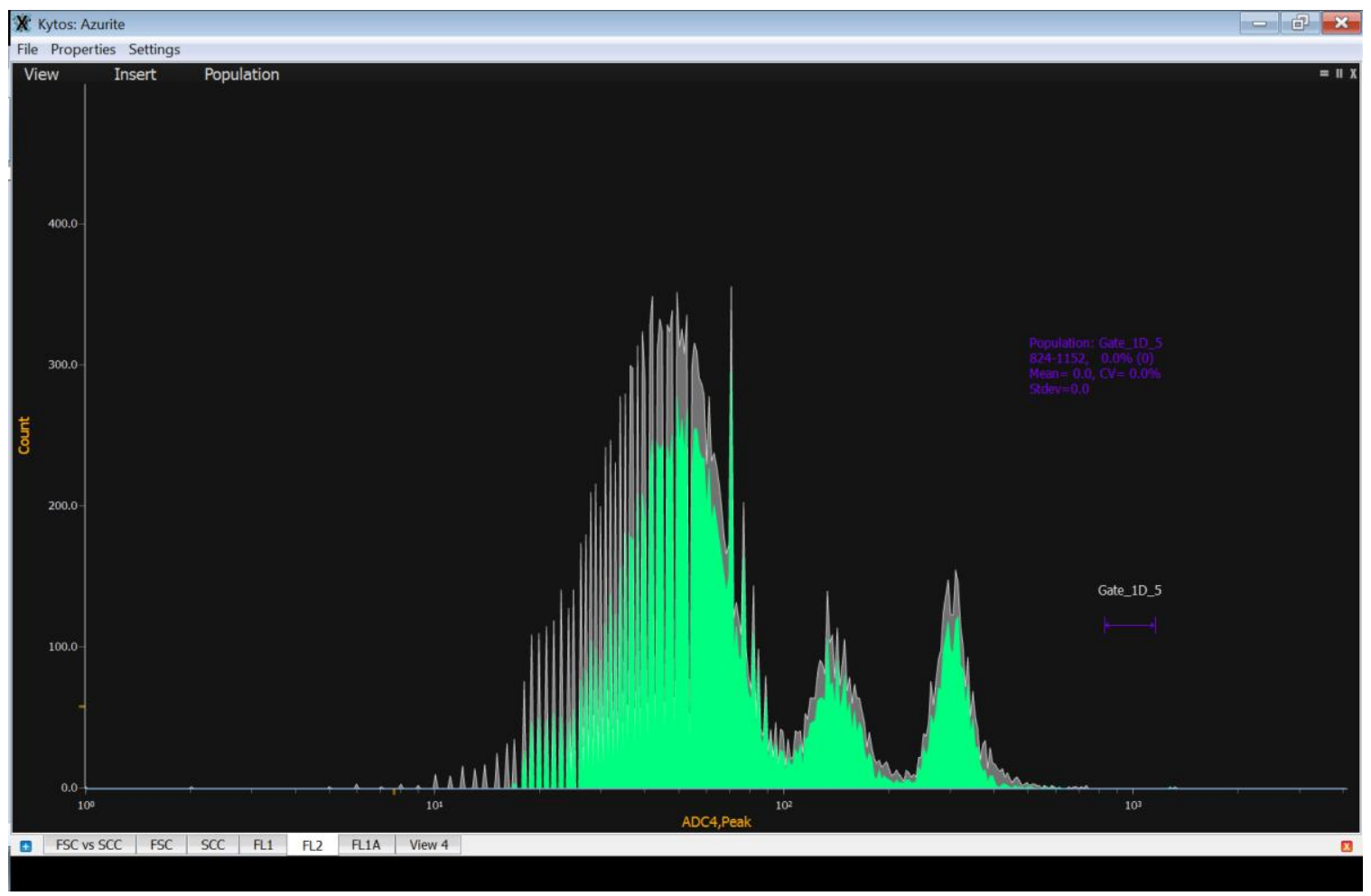

FL2 200mm focal lens 


\section{Plano Convex Beam Shaping Optic}

This Appendix contains specifications on the $200 \mathrm{~mm}$ focal length plano convex lens from ThorLabs. This lens is used for shaping the beam of the laser in the Y plane. 


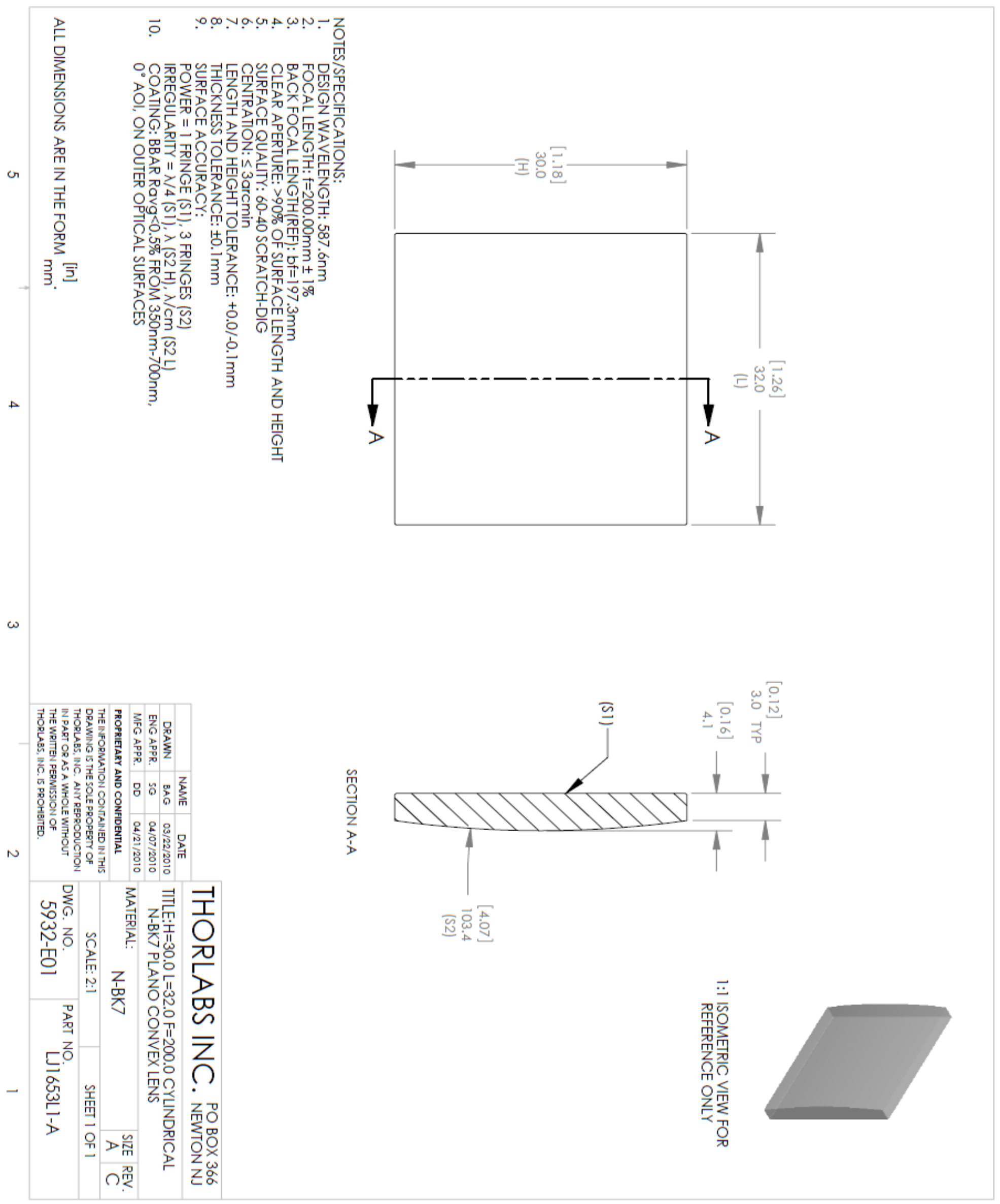


J. Plano Convex Beam Shaping Optic

This Appendix contains specification on the $40 \mathrm{~mm}$ focal length plano covex lens from ThorLabs. This lens is used for shaping the laser beam in the X plane. 


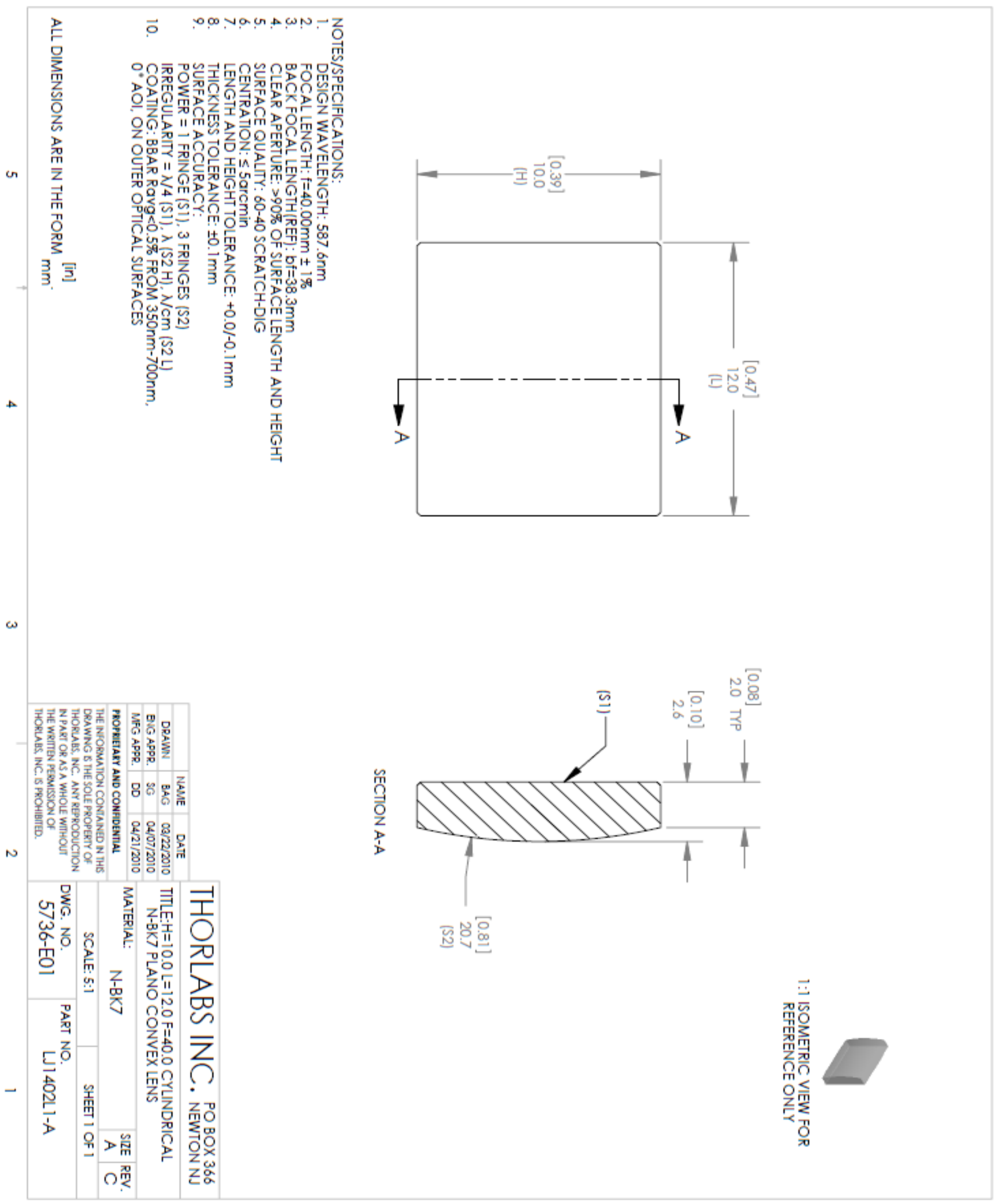


K. Flow Cell Focal Lens

This Appendix contains specification on the $8 \mathrm{~mm}$ focal length lens from Edmund Optics. This lens was used to focus the correct beam shape onto the capillary. The lens was mounted inside the flow cell approximately $8 \mathrm{~mm}$ for the capillary. 
TECHSPEC $4 \mathrm{~mm}$ Dia. $x 8 \mathrm{~mm}$ FL, $\mathrm{MgF}_{2}$ Coated, Achromatic Doublet Lens

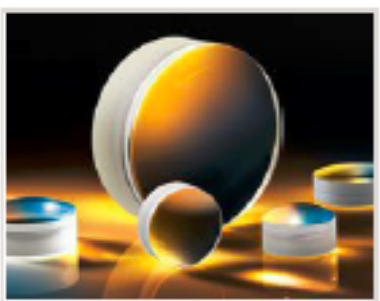

Avallability: In smoox

$\$ 70.00$

$\frac{1}{1}-5$ for $\$ 70.00$ esch.
$6=25$ for $\$ 56.00$ esch.

SPECIFICATIONS

\begin{tabular}{l|l} 
Dismeter $(\mathrm{mm})$ & 4.00
\end{tabular}

\begin{tabular}{|l|l|l|l}
\hline Dismeter Tolerance $(\mathrm{mm})$ & $+0.00 /-0.05$
\end{tabular}

\begin{tabular}{|l|l|}
\hline Clesr Aperture CA $(\mathrm{mm})$ & 3.6
\end{tabular}

\begin{tabular}{l|l} 
Effoctive Focal Length EFL (mm) & 8.0
\end{tabular}

\begin{tabular}{|l|l|l|l}
\hline Back Focal Length BFL. $(\mathrm{mm})$ & 6.69
\end{tabular}

\begin{tabular}{|l|l} 
Focal Length Tolerance ( $\%)$ & \pm 2
\end{tabular}

\begin{tabular}{|l|l}
\hline Edge Thickness ET $(\mathrm{mm})$ & 2.09
\end{tabular}

\begin{tabular}{l|l} 
Center Thidcness CT $1(\mathrm{~mm})$ & 1.50
\end{tabular}

\begin{tabular}{|l|l}
\hline Center Thidcness CT $2(\mathrm{~mm})$ & 1.03
\end{tabular}

\begin{tabular}{l|l} 
Center Thickness Tolerance $(\mathrm{mm})$ & \pm 0.2
\end{tabular}

\begin{tabular}{|l|l|}
\hline Centering (arcminutes) & $3-5$
\end{tabular}

\begin{tabular}{l|l} 
Radlus $\mathrm{R}_{1}(\mathrm{~mm})$ & 5.76
\end{tabular}

\begin{tabular}{|l|l|l|l|l}
\hline Radius $\mathrm{R}_{2}(\mathrm{~mm})$ & -3.49
\end{tabular}

\begin{tabular}{|l|l|l|l}
\hline Radius $R_{3}(\mathrm{~mm})$ & -26.05
\end{tabular}

\begin{tabular}{l|l}
\hline Surface Quality & $20-10$
\end{tabular}

Bevel

Substrate

Protective bevel as needed N-BaF10/ N-SF10

\begin{tabular}{l|l} 
Coating Specification & $R_{\text {avg }} \leq 1.75 \%$ 0 $400-700 \mathrm{~nm}$
\end{tabular}

\begin{tabular}{l|l} 
Costing & $\mathrm{MgF}_{2}$
\end{tabular}

Type

$\mathrm{t} / \mathrm{W}$

\begin{tabular}{|l|l}
\hline Numerical Aperture NA & 0.25
\end{tabular}

\begin{tabular}{|l|l|l} 
Wavelength Range (jum) & $0.4-0.7$
\end{tabular}

RoHs

TECHNICAL INFORMATION

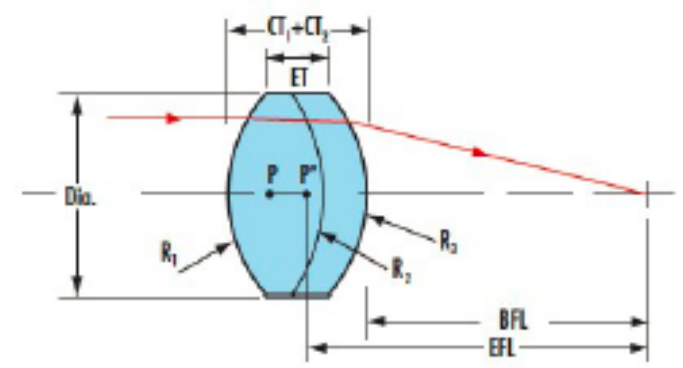




\section{Assembly of Machine}

The images in this Appendix are final pictures taken of the CytoFlow device. These show the whole cabinet that contains the device as well as a picture of the optics layout. 


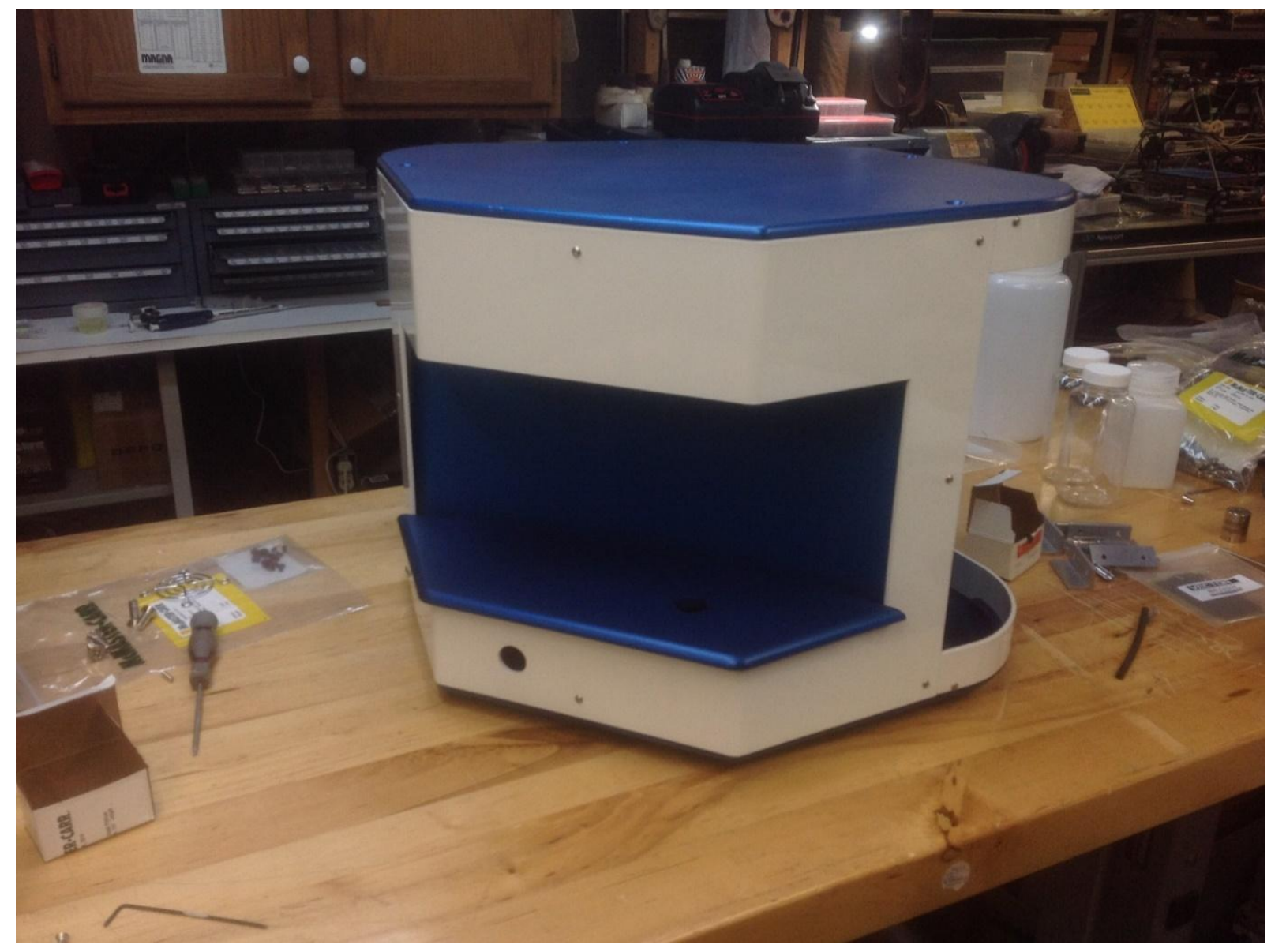

The Image above displays the shell that the complete flow cell is housed inside. 


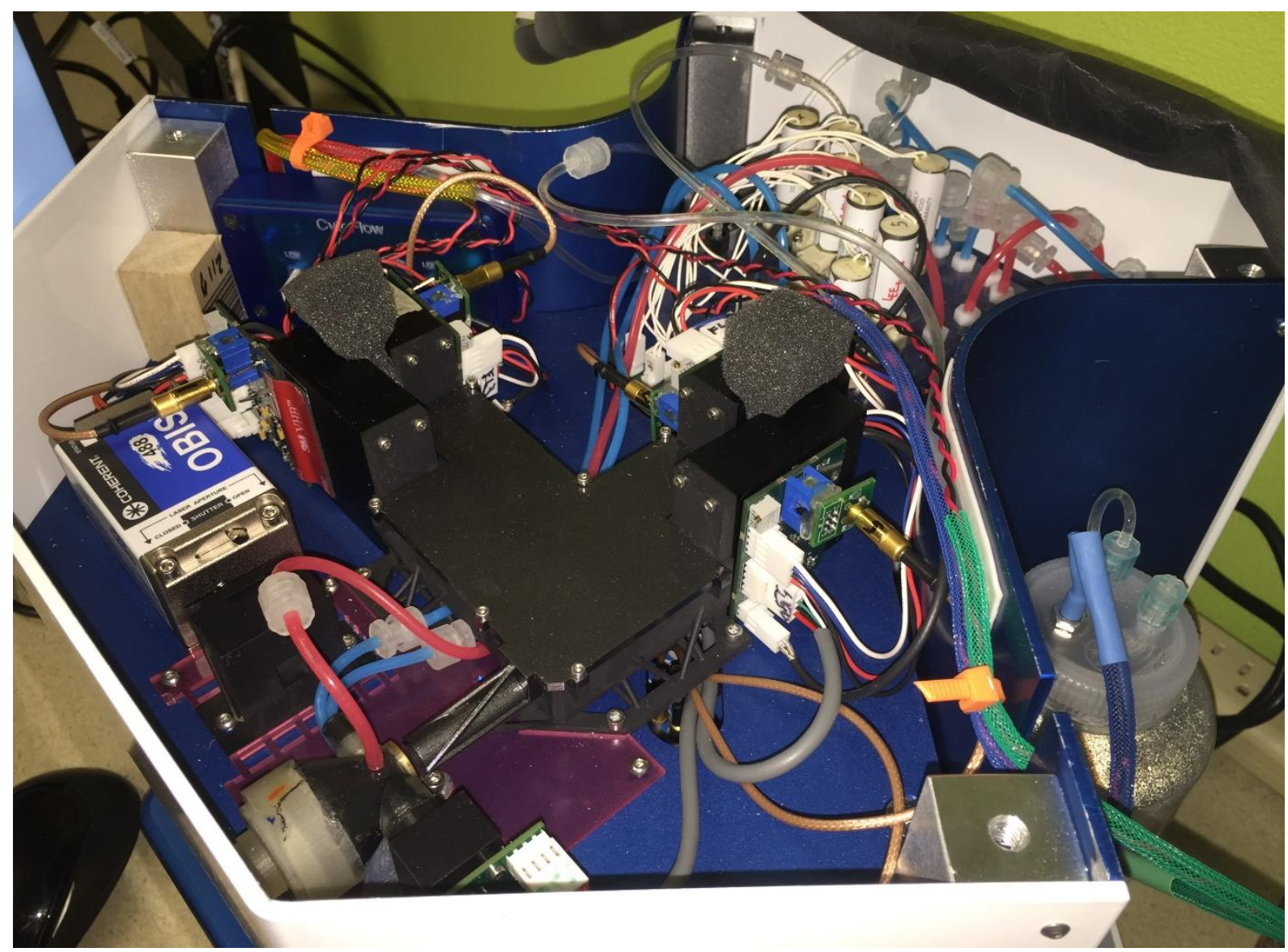

The image above is a real picture taken of the optics portion of the machine. This image shows all of the detail that went into the device created by CytoFlow. 